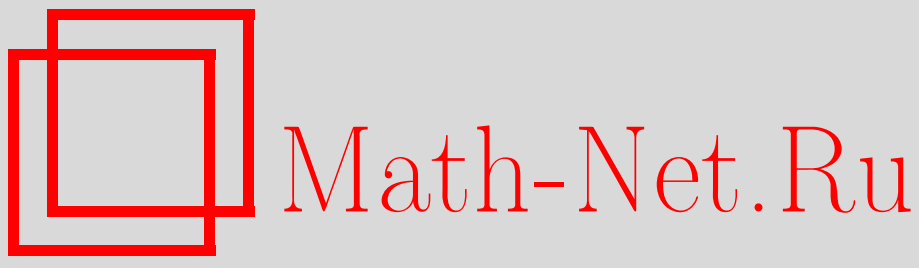

Г. В. Сандраков, Осреднение параболических уравнений с контрастными коэффициентами, Изв. РАН. Сер. матем., 1999, том 63, выпуск 5, 179-224

DOI: https://doi.org/10.4213/im264

Использование Общероссийского математического портала Math-Net.Ru подразумевает, что вы прочитали и согласны с пользовательским соглашением http://www . mathnet.ru/rus/agreement

Параметры загрузки:

IP : 54.224 .187 .69

26 апреля 2023 г., $10: 51: 34$ 
УДК 517.9

Г.В. Сандраков

\title{
Осреднение параболических уравнений с контрастными коэффициентами
}

\begin{abstract}
Рассматривается нестационарная задача диффузии в периодической среде с включениями, заполненными слабопроводящим материалом. Получены осредненные уравнения, приближающие решения рассматриваемой задачи. Доказаны оценки точности такого приближения при стремлении к нулю периода среды и коэффициента проводимости. Вид осредненных уравнений и оценки точности существенно зависят от предельного поведения коэффициента проводимости в сравнении с квадратом периода.
\end{abstract}

Библиограффия: 20 наименований.

\section{§1. Введение}

В работе рассматриваются нестационарные уравнения параболического типа с коэффициентами, зависящими от двух малых положительных параметров $\varepsilon$ и $\sigma$. Параметр мелкомасштабности $\varepsilon$ задает период коэффищиентов таких уравнений, а параметр контрастности $\sigma$ характеризует отношение минимального и максимального значений коэффициентов проводимости. Начально-краевые задачи для этих уравнений моделируют диффузию в среде с включениями, имеюшими малую проводимость и расположенными периодическим образом с периодом $\varepsilon$. Предполагается, что при $\varepsilon \rightarrow 0$ выполнены соотношение $\sigma \rightarrow 0$ и одно из следуюших трех условий:

$$
\begin{aligned}
& \frac{\sigma}{\varepsilon^{2}} \rightarrow \infty \\
& \frac{\sigma}{\varepsilon^{2}} \rightarrow \vartheta \\
& \frac{\sigma}{\varepsilon^{2}} \rightarrow 0,
\end{aligned}
$$

где $\vartheta$ - заданная положительная постоянная. Будут получены осредненные (предельные) уравнения, решения которых приближают решения рассматриваемых уравнений, и доказаны оценки точности такого приближения.

Пусть для $n \geqslant 2$ заданы ограниченная область $\Omega \subset \mathbb{R}^{n}$ с гладкой (класса $\left.C^{\infty}\right)$ границей, положительное $T$, финитные в $\Omega$ игладкие функции $f, g \in C_{0}^{\infty}([0, T] \times \Omega)$ и $w \in C_{0}^{\infty}(\Omega)$. Определим функцию $u$ как решение начально-краевой задачи

$$
\begin{gathered}
m_{\varepsilon} u_{t}^{\prime}-\operatorname{div} A_{\varepsilon}^{\sigma}\left(\nabla u+a_{\varepsilon} u+b_{\varepsilon} g\right)+k_{\varepsilon}^{\sigma} \nabla u=r_{\varepsilon} f \quad \text { в } \Omega \times(0, T), \\
u=0 \text { на } \partial \Omega \times(0, T),\left.\quad u\right|_{t=0}=w \text { в } \Omega,
\end{gathered}
$$

зависяшей от малых положительных параметров $\varepsilon$ и $\sigma$, следующим образом.

Работа выполнена при финансовой поддержке Российского фонда фундаментальных исследований (грант № 96-01-01394) и INTAS (грант № 96-1061). 
Пусть $F_{1}$ является 1-периодическим открытым связным подмножеством $\mathbb{R}^{n}$ с границей класса $C^{2}, F_{0}=\mathbb{R}^{n} \backslash \bar{F}_{1}$ является (не обязательно связным) множеством с границей класса $C^{2}$ и $F_{\varepsilon}=\varepsilon F_{1}=\left\{\varepsilon x, x=\left(x_{1}, \ldots, x_{n}\right) \in F_{1}\right\}$. Здесь и далее 1-периодичность множества (функции) означает периодичность характеристической функции этого множества (этой функции) с периодом 1 по каждому $x_{k}, k=1, \ldots, n$. Таким образом, $Y=[0,1]^{n}$ является ячейкой периодичности и множества $F_{1}, F_{0}$ вполне определяются множествами $Y_{1}=Y \cap F_{1}$ и $Y_{0}=Y \cap F_{0}$. Предполагается, что эти множества имеют положительные меры Лебега в $\mathbb{R}^{n}$ :

$$
\theta_{0}=\operatorname{mes}\left(Y_{0}\right)>0, \quad \theta_{1}=\operatorname{mes}\left(Y_{1}\right)>0 .
$$

Кроме того, отождествляя противоположные грани $Y$, множества $Y_{1}$ и $Y_{0}$ можно также рассматривать как подмногообразия тора с общей границей (класса $C^{2}$ ): $\partial Y_{1}=\partial Y_{0} \equiv S$.

Матричнозначная функция $A_{\varepsilon}^{\sigma}$ и вектор-функция $k_{\varepsilon}^{\sigma}$ в (1.4) зависят от $\varepsilon$ и $\sigma$ :

$$
\begin{aligned}
& A_{\varepsilon}^{\sigma}=A_{1}\left(\frac{x}{\varepsilon}\right), \quad k_{\varepsilon}^{\sigma}=k_{1}\left(\frac{x}{\varepsilon}\right) \quad \text { в } \quad \Omega_{1}^{\varepsilon}=\Omega \cap F_{\varepsilon}, \\
& A_{\varepsilon}^{\sigma}=\sigma A_{0}\left(\frac{x}{\varepsilon}\right), \quad k_{\varepsilon}^{\sigma}=\sigma k_{0}\left(\frac{x}{\varepsilon}\right) \quad \text { в } \quad \Omega_{0}^{\varepsilon}=\Omega \backslash \bar{\Omega}_{1}^{\varepsilon} \text {. }
\end{aligned}
$$

Предполагается, что компоненты матричнозначных функций $A_{1}(y), A_{0}(y)$ и вектор-функций $k_{1}(y), k_{0}(y)$ являются 1-периодическими ограниченньгми (почти всюду) функциями на $F_{1}$ и $F_{0}$ соответственно. Кроме того, $A_{1}(y)$ и $A_{0}(y)$ симметричны и равномерно эллиптичны:

$$
\begin{array}{ll}
\alpha|\xi|^{2} \leqslant\left(A_{1}(y) \xi, \xi\right) \leqslant \beta|\xi|^{2}, & \left|k_{1}(y)\right| \leqslant \beta \text { для } y \in Y_{1} \text { и } \xi \in \mathbb{R}^{n}, \\
\alpha|\xi|^{2} \leqslant\left(A_{0}(y) \xi, \xi\right) \leqslant \beta|\xi|^{2}, & \left|k_{0}(y)\right| \leqslant \beta \quad \text { для } y \in Y_{0} \text { и } \xi \in \mathbb{R}^{n},
\end{array}
$$

где $\alpha$ и $\beta$ - заданные положительные постоянные и $(\cdot, \cdot)$ обозначает скалярное произведение векторов из $\mathbb{R}^{n}$. Функции $m_{\varepsilon}$ и $r_{\varepsilon}$ в $(1.4)$ зависят от $\varepsilon$ :

$$
\begin{array}{llll}
m_{\varepsilon}=m_{1}\left(\frac{x}{\varepsilon}\right), & r_{\varepsilon}=r_{1}\left(\frac{x}{\varepsilon}\right) \text { в } \Omega_{1}^{\varepsilon}, \\
m_{\varepsilon}=m_{0}\left(\frac{x}{\varepsilon}\right), & r_{\varepsilon}=r_{0}\left(\frac{x}{\varepsilon}\right) \text { в } \Omega_{0}^{\varepsilon},
\end{array}
$$

где $m_{1}(y), r_{1}(y)$ и $m_{0}(y), r_{0}(y)$ являются 1-периодическими ограниченными функциями на $F_{1}$ и $F_{0}$. Кроме того, $m_{1}(y)$ и $m_{0}(y)$ отделены от нуля:

$$
\begin{array}{ll}
\alpha \leqslant m_{1}(y) \leqslant \beta, & \left|r_{1}(y)\right| \leqslant \beta \text { для } \quad y \in Y_{1}, \\
\alpha \leqslant m_{0}(y) \leqslant \beta, & \left|r_{0}(y)\right| \leqslant \beta \quad \text { для } \quad y \in Y_{0} .
\end{array}
$$

Вектор-функции $a_{\varepsilon}$ и $b_{\varepsilon}$ в $(1.4)$ зависят от $\varepsilon$ :

$$
\begin{array}{lll}
a_{\varepsilon}=a_{1}\left(\frac{x}{\varepsilon}\right), & b_{\varepsilon}=b_{1}\left(\frac{x}{\varepsilon}\right) \quad \text { в } \Omega_{1}^{\varepsilon}, \\
a_{\varepsilon}=a_{0}\left(\frac{x}{\varepsilon}\right), & b_{\varepsilon}=b_{0}\left(\frac{x}{\varepsilon}\right) \quad \text { в } \quad \Omega_{0}^{\varepsilon},
\end{array}
$$

где компоненты вектор-функций $a_{1}(y), b_{1}(y)$ и $a_{0}(y), b_{0}(y)$ являются 1 -периодическими ограниченными функциями на $F_{1}$ и $F_{0}$. 
Таким образом, не зависящие от времени быстроосциллируюшие коэффициенты первого уравнения в (1.4) имеют единичный порядок на множестве $\Omega_{1}^{\varepsilon}$ и частично на множестве $\Omega_{0}^{\varepsilon}$, но коэффициенты проводимости $A_{\varepsilon}^{\sigma}$ и переноса $k_{\varepsilon}^{\sigma}$ малы на $\Omega_{0}^{\varepsilon}$. Кроме того, малость этих коэффициентов контролируется одним из предположений (1.1)-(1.3), а на множества $\Omega_{1}^{\varepsilon}$ и $\Omega_{0}^{\varepsilon}$ наложены условия непустоты (1.5) и некоторой регулярности "внутренних" границ.

Поскольку коэффициенты в (1.4) могут быть разрывньми, следуя [1], решение этой задачи определим как элемент $u \in L^{2}\left(0, T ; H_{0}^{1}(\Omega)\right)$, удовлетворяющий для каждого $\varphi \in H_{0}^{1}(\Omega)$ и почти всех $t \in(0, T)$ интегральному тождеству

$$
\int_{\Omega} m_{\varepsilon} u_{t}^{\prime} \varphi d x+\int_{\Omega}\left(A_{\varepsilon}^{\sigma}\left(\nabla u+a_{\varepsilon} u+b_{\varepsilon} g\right), \nabla \varphi\right) d x+\int_{\Omega} k_{\varepsilon}^{\sigma} \nabla u \varphi d x=\int_{\Omega} r_{\varepsilon} f \varphi d x
$$

и начальному условию $\left.u\right|_{t=0}=w$ в $L^{2}(\Omega)$. Известно [1], что для фиксированных $\varepsilon$ и $\sigma$ сушествует единственное решение этой задачи такое, что $u \in C^{0}\left([0, T] ; L^{2}(\Omega)\right)$ и $u_{t}^{\prime} \in L^{2}\left(0, T ; L^{2}(\Omega)\right)$. Здесь используются функциональные пространства, определенные, например, в [1].

Основные результаты работы составляют теоремы о предельном поведении решений задачи (1.4) при $\varepsilon \rightarrow 0$ в предположении, что вьполнены соотношение $\sigma \rightarrow 0$ и одно из условий (1.1)-(1.3). Для точной формулировки этих утверждений понадобятся некоторые дополнительные обозначения.

Пусть вектор-функция $N_{1}(y)$ является 1-периодическим решением задачи Неймана на $Y_{1}$ :

$$
-\operatorname{div}_{y}\left(A_{1} \nabla_{y} N_{1}\right)=\operatorname{div}_{y} A_{1} \quad \text { в } Y_{1}, \quad-\left(A_{1} \nabla_{y} N_{1}, \nu\right)=\left(A_{1}, \nu\right) \text { на } S,
$$

где $\nu$ - внешняя нормаль к границе $Y_{1}$, и использованы обозначения (1.6). Определим матрицу $A$ с постоянными компонентами равенством

$$
A=\left\langle A_{1}+A_{1} \nabla_{y} N_{1}\right\rangle_{\mathbf{1}} \equiv \int_{Y_{1}}\left(A_{1}+A_{1} \nabla_{y} N_{1}\right) d y .
$$

Известно (см. [2], [3]), что матрица $A$ положительно определена.

Пусть функции $N_{a}(y), N_{b}(y)$ являются 1-периодическими решениями задач Неймана на $Y_{1}$ :

$$
\begin{aligned}
& -\operatorname{div}_{y}\left(A_{1} \nabla_{y} N_{a}\right)=\operatorname{div}_{y}\left(A_{1} a_{1}\right) \text { в } Y_{1}, \quad-\left(A_{1} \nabla_{y} N_{a}, \nu\right)=\left(A_{1} a_{1}, \nu\right) \text { на } S, \\
& -\operatorname{div}_{y}\left(A_{1} \nabla_{y} N_{b}\right)=\operatorname{div}_{y}\left(A_{1} b_{1}\right) \text { в } Y_{1}, \quad-\left(A_{1} \nabla_{y} N_{b}, \nu\right)=\left(A_{1} b_{1}, \nu\right) \text { на } S,
\end{aligned}
$$

где использованы обозначения (1.6) и (1.10). Далее, учитывая (1.6), (1.8) и (1.13), введем обозначения

$$
\begin{aligned}
M & =\left\langle m_{0}\right\rangle_{\mathbf{0}}+\left\langle m_{1}\right\rangle_{\mathbf{1}} \equiv \int_{Y_{0}} m_{0} d y+\int_{Y_{1}} m_{1} d y, \quad R=\left\langle r_{0}\right\rangle_{\mathbf{0}}+\left\langle r_{1}\right\rangle_{\mathbf{1}}, \\
a & =A^{-1}\left\langle A_{1} a_{1}+A_{1} \nabla_{y} N_{a}\right\rangle_{\mathbf{1}}, \quad b=A^{-1}\left\langle A_{1} b_{1}+A_{1} \nabla_{y} N_{b}\right\rangle_{\mathbf{1}}, \\
K & =\left\langle k_{1}+k_{1} \nabla_{y} N_{1}\right\rangle_{\mathbf{1}}, \quad k_{a}=\left\langle k_{1} \nabla_{y} N_{a}\right\rangle_{\mathbf{1}}, \quad k_{b}=\left\langle k_{1} \nabla_{y} N_{b}\right\rangle_{\mathbf{1}} .
\end{aligned}
$$

Определим функцию $v$ как решение начально-краевой задачи для осредненного уравнения с постоянными коэффициентами:

$$
\begin{gathered}
M v_{t}^{\prime}-\operatorname{div} A(\nabla v+a v+b g)+K \nabla v+k_{a} v+k_{b} g=R f \quad \text { в } \Omega \times(0, T), \\
v=0 \text { на } \partial \Omega \times(0, T),\left.\quad v\right|_{t=0}=w \text { в } \Omega,
\end{gathered}
$$

где использованы обозначения (1.13) и (1.15). 
Теорема 1. Пусть выполнено условие (1.1). Предположим, что и является решением задачи (1.4) и v-решение задачи (1.16). Тогда

$$
\max _{0 \leqslant t \leqslant T}\|u-v\|_{L^{2}(\Omega)}^{2} \leqslant C\left(\varepsilon+\sigma+\frac{\varepsilon^{2}}{\sigma}\right),
$$

где постоянная $C$ не зависит от $\varepsilon$ и $\sigma$.

Пусть функции $Q(t, y)$ и $P(t, y)$ являются 1-периодическими решениями начально-краевых задач на $Y_{0}$ :

$$
\begin{aligned}
& m_{0} Q_{t}^{\prime}-\vartheta \operatorname{div}_{y}\left(A_{0} \nabla_{y} Q\right)=0 \text { в } Y_{0} \times(0, \infty), \\
& Q=0 \text { на } \bar{Y}_{1} \times(0, \infty),\left.\quad Q\right|_{t=0}=1 \text { в } Y_{0}, \\
& m_{0} P_{t}^{\prime}-\vartheta \operatorname{div}_{y}\left(A_{0} \nabla_{y} P\right)=0 \text { в } Y_{0} \times(0, \infty), \\
& P=0 \text { на } \bar{Y}_{1} \times(0, \infty),\left.\quad P\right|_{t=0}=\frac{r_{0}}{m_{0}} \text { в } Y_{0},
\end{aligned}
$$

где учтены обозначения (1.2), (1.6) и (1.8). Далее введем обозначения

$$
\begin{array}{rlrl}
M_{1} & =\left\langle m_{1}\right\rangle_{\mathbf{1}}, & M_{0}(t)=\left\langle m_{0} Q_{t}^{\prime}\right\rangle_{\mathbf{0}}, & Q_{\varepsilon}=Q\left(t, \frac{x}{\varepsilon}\right), \\
R_{1}=\left\langle r_{1}\right\rangle_{\mathbf{1}}, & R_{0}(t)=\left\langle m_{0} P_{t}^{\prime}\right\rangle_{\mathbf{0}}, & P_{\varepsilon}=P\left(t, \frac{x}{\varepsilon}\right)
\end{array}
$$

(здесь, например, $Q(t, y)$ продолжена как 1-периодическая функция на $\mathbb{R}^{n}$, тогда $Q_{\varepsilon}$ обозначает ограничение этого продолжения при $y=x / \varepsilon$ на $\Omega$ ) и определим функцию $v$ как решение начально-краевой задачи для осредненного уравнения

$$
\begin{aligned}
& M_{1} v_{t}^{\prime}-M_{0} *\left(v_{t}^{\prime}\right)-\operatorname{div} A(\nabla v+a v+b g)+K \nabla v+k_{a} v \\
& \quad+k_{b} g=R_{1} f-R_{0} * f \text { в } \Omega \times(0, T), \\
& v=0 \text { на } \partial \Omega \times(0, T),\left.\quad v\right|_{t=0}=w \text { в } \Omega
\end{aligned}
$$

где $*$ обозначает свертку по $t$ и использованы обозначения (1.13) и (1.15).

Теорема 2. Пусть выполнено условие (1.2), $f, g \in C_{0}^{\infty}((0, T) \times \Omega) u w=0$. Предположим, что и является решением задачи (1.4) и $v$-решение задачи (1.19). Тогда

$$
\begin{aligned}
\max _{0 \leqslant t \leqslant T} & \left\|u-v-P_{\varepsilon} * f+Q_{\varepsilon} *\left(v_{t}^{\prime}\right)\right\|_{L^{2}(\Omega)}^{2} \\
& \leqslant c\left(\varepsilon+\varepsilon \sqrt{\vartheta}+\sigma+\frac{\sigma}{\varepsilon \sqrt{\vartheta}}+\frac{\varepsilon}{\sqrt{\sigma \vartheta}}\left|\vartheta-\frac{\sigma}{\varepsilon^{2}}\right|\right) \\
& \leqslant C\left(\varepsilon+\sqrt{\sigma}+\left|\vartheta-\frac{\sigma}{\varepsilon^{2}}\right|\right),
\end{aligned}
$$

где постоянные $C$ и с не зависят от $\varepsilon, \sigma$ ис не зависит от $\vartheta$.

Пусть $\chi_{1}^{\varepsilon}$ и $\chi_{0}^{\varepsilon}$ обозначают характеристические функции областей $\Omega_{1}^{\varepsilon}$ и $\Omega_{0}^{\varepsilon}$ соответственно. Определим функцию $v$ как решение начально-краевой задачи для осредненного уравнения с постоянными коэффициентами:

$$
\begin{gathered}
M_{1} v_{t}^{\prime}-\operatorname{div} A(\nabla v+a v+b g)+K \nabla v+k_{a} v+k_{b} g=R_{1} f \quad \text { в } \Omega \times(0, T), \\
v=0 \text { на } \partial \Omega \times(0, T),\left.\quad v\right|_{t=0}=w \quad \text { в } \Omega,
\end{gathered}
$$

где использованы обозначения (1.13), (1.15) и (1.18). 
ТеОрема 3. Пусть выполнено условие (1.3), $m_{0}, r_{0} \in C^{2}\left(F_{0}\right), \quad A_{0} \in$ $C^{2}\left(F_{0}\right)^{n \times n}$ u $F_{0} \in C^{3}$. Предположим, что $u$-решение задачи (1.4) и $v$ решение задачи (1.20). Тогда

$$
\max _{0 \leqslant t \leqslant T}\left\|u-\chi_{1}^{\varepsilon} v-\chi_{0}^{\varepsilon} w-\chi_{0}^{\varepsilon}\left(\frac{r_{0}}{m_{0}}\right)_{\varepsilon} \int_{0}^{t} f d t\right\|_{L^{2}(\Omega)}^{2} \leqslant C\left(\varepsilon+\sqrt{\sigma}+\frac{\sqrt{\sigma}}{\varepsilon}\right),
$$

где интеграл обозначает первообразную по $t u C$ не зависит от $\varepsilon u \sigma$.

ЗАмЕчАнИЕ 1 . Проверяется, что в условиях теорем 1-3 достаточно гладкие решения осредненных задач (1.16), (1.19), (1.20) существуют и определены однозначно. Гладкость и финитность $f, g, w$ (и равенство $w=0$ из условий теоремы 2) используются в доказательстве именно этого утверждения о решениях осредненных задач. Это утверждение для задач (1.16) и (1.20) хорошо известно [4], поскольку при сделанных предположениях на исходные данные выполнены условия согласования произвольного порядка. Это утверждение для задачи (1.19) выводится в 2 из результатов работы [6], в которой была доказана разрешимость задачи (1.19) в пространстве $H^{1}\left(0, T ; L^{2}(\Omega)\right) \cap L^{2}\left(0, T ; H_{0}^{1}(\Omega)\right)$.

ЗАмечАниЕ 2. Для построения начальных членов асимптотического разложения решения задачи (1.4) использовались методы работ [5] и [7]. Это построение заключается в последовательном разрешении некоторых периодических задач на ячейке (типа задач $(1.12),(1.14)$ ), условия разрешимости которых приводят к осредненным задачам $(1.16),(1.19)$ или (1.20) в зависимости от вьполнения одного из условий (1.1)-(1.3). В данной работе приводятся явные формулы этих разложений. После этого доказательство теорем 1-3 проводится энергетическими методами. При этом учитывается гладкость решений осредненных задач (на самом деле конечного порядка; см. §2) и некоторая регулярность решений задач на ячейке, в доказательстве которой используется гладкость (класса $C^{2}$ ) границы $S$. Кроме того, в случае выполнения условия (1.3) эту асимптотику необходимо "подправить" погранслойными добавками, экспоненциально убывающими внутрь включений. Эти добавки строятся в $\S 6$ по схеме метода Вишика-Люстерника. Для задач осреднения эта схема реализована в работе [5]. Гладкость коэффициентов и границы включений из условий теоремы 3 используются при построении этих погранслойных добавок. В реальных задачах, встречающихся на практике, коэффициенты $m_{1}, m_{0}, A_{1}, A_{0}, k_{1}, \ldots$ как правило постоянны, так что гладкость коэффициентов не слишком существенна. Кроме того, предположение о постоянстве коэффициентов приводит к упрощениям некоторых формул. Например, если компоненты вектор-функций $a_{1}$ и $b_{1}$ в (1.10) постоянны, то $N_{a}=N_{1} a_{1}, N_{b}=N_{1} b_{1}$, и поэтому $a=a_{1}, b=b_{1}$ в формулах (1.15).

ЗАмЕЧАнИЕ 3. Вопросы осреднения стационарного варианта задачи (1.4) рассматривались в работах [2], [8] и [9]. Задача (1.4) в случае, когда $\sigma=\varepsilon^{2}$, и в некоторых отличных, чем здесь, предположениях рассматривалась в работах [6] и [10], в которых были приведены утверждения о сходимости решений в соответствующих пространствах. Отметим также, что, используя подходящие аппроксимации исходных данных, из теорем 1-3 несложно вывести утверждения о сходимости решений задачи (1.4) в норме пространства $L^{\infty}\left(0, T ; L^{2}(\Omega)\right)$ при более слабых предположениях на $f, g$ и $w$. Точнее, в обозначениях из теорем 1-3 выполнены следующие утверждения о сходимости решений. 
СлЕДСТВИЕ 1. Пусть выполнено условие (1.1) и $f, g \in L^{2}\left(0, T ; L^{2}(\Omega)\right), w \in$ $L^{2}(\Omega)$ в задаче (1.4). Предположим, что и-решение задачи (1.4) и v-решение задачи (1.16). Тогда

$$
\|u-v\|_{L^{\infty}\left(0, T ; L^{2}(\Omega)\right)} \rightarrow 0
$$

$n p u \varepsilon \rightarrow 0, \quad \varepsilon^{2} / \sigma \rightarrow 0, \quad \sigma \rightarrow 0$.

СлЕДСТВИЕ 2. Пусть выполнено условие (1.2) и $f \in L^{2}\left(0, T ; L^{2}(\Omega)\right)$, $g \in L^{2}\left(0, T ; H_{0}^{1}(\Omega)\right), w=0$ в задаче (1.4). Предположим, что и-решение задачи (1.4) и v-решение задачи (1.19). Тогда

$$
\|u-v\|_{L^{\infty}\left(0, T ; L^{2}\left(\Omega_{1}^{\varepsilon}\right)\right)}+\left\|u-v-P_{\varepsilon} * f+Q_{\varepsilon} *\left(v_{t}^{\prime}\right)\right\|_{L^{\infty}\left(0, T ; L^{1}\left(\Omega_{0}^{\varepsilon}\right)\right)} \rightarrow 0
$$

$n p u \varepsilon \rightarrow 0, \quad \sigma / \varepsilon^{2} \rightarrow \vartheta, \quad \sigma \rightarrow 0$.

СлЕДСТВИЕ 3. Пусть выполнено условие (1.3), $m_{0}, r_{0} \in C^{2}\left(F_{0}\right), A_{0} \in$ $C^{2}\left(F_{0}\right)^{n \times n}, \quad \partial F_{0} \in C^{3} u \quad f, g \in L^{2}\left(0, T ; L^{2}(\Omega)\right), \quad w \in L^{2}(\Omega)$ в задаче (1.4). Предположим, что и - решение задачи (1.4) и $v$-решение задачи (1.20). Тогда

$$
\left\|u-\chi_{1}^{\varepsilon} v-\chi_{0}^{\varepsilon} w-\chi_{0}^{\varepsilon}\left(\frac{r_{0}}{m_{0}}\right)_{\varepsilon} \int_{0}^{t} f d t\right\|_{L^{\infty}\left(0, T ; L^{2}(\Omega)\right)} \rightarrow 0
$$

$n p u \varepsilon \rightarrow 0, \quad \sigma / \varepsilon^{2} \rightarrow 0, \quad \sigma \rightarrow 0$.

ЗАмЕчАниЕ 4. В реальных задачах, встречающихся на практике, имеются разнообразные асимптотические соотношения между коэффициентами задачи (1.4). Некоторые из этих соотношений приведены в работах [11], [12] и отличаются от принятых здесь. В работе [12] рассмотрена задача (1.4) в случае, когда $\sigma=\varepsilon^{2}, k_{\varepsilon}^{\sigma}=0$ и в условии (1.10) коэффициенты $a_{0}(y)$ и $b_{0}(y)$ умножены на $\varepsilon^{-1}$. При этих предположениях в [12] доказана сходимость решений задачи (1.4) к решению осредненной задачи в пространстве $L^{2}\left(0, T ; L^{2}(\Omega)\right)$. Однако осредненная задача в [12] определяется через решение "двухмасштабной задачи на ячейке", зависящей от параметра $x \in \Omega$. Используемые здесь методы позволяют "разделить" масштабы в этой задаче и уточнить результаты работы [12] следующим образом.

Предположим, что в (1.6) и (1.10) определения коэффициентов $k_{\varepsilon}^{\sigma}, a_{\varepsilon}$ и $b_{\varepsilon}$ в области $\Omega_{0}^{\varepsilon}$ заменены более обшими равенствами:

$$
\begin{array}{ll}
k_{\varepsilon}^{\sigma}=\sigma k_{0}\left(\frac{x}{\varepsilon}\right)+\sqrt{\sigma} k_{0}^{0}\left(\frac{x}{\varepsilon}\right) \quad \text { в } \quad \Omega_{0}^{\varepsilon}, \\
a_{\varepsilon}=a_{0}\left(\frac{x}{\varepsilon}\right)+a_{0}^{0}\left(\frac{x}{\varepsilon}\right) \frac{1}{\sqrt{\sigma}}, \quad b_{\varepsilon}=b_{0}\left(\frac{x}{\varepsilon}\right)+b_{0}^{0}\left(\frac{x}{\varepsilon}\right) \frac{1}{\sqrt{\sigma}} \text { в } \Omega_{0}^{\varepsilon},
\end{array}
$$

где компоненты вектор-функций $k_{0}(y), k_{0}^{0}(y), a_{0}(y), a_{0}^{0}(y), b_{0}(y)$ и $b_{0}^{0}(y)$ являются 1 -периодическими ограниченными функциями на $F_{0}$. Предположим также, что 
$\operatorname{div}_{y}\left(A_{0} a_{0}^{0}\right), \operatorname{div}_{y}\left(A_{0} b_{0}^{0}\right), \operatorname{div}_{y}\left(k_{0}^{0}\right) \in L^{\infty}\left(Y_{0}\right)$. Пусть функции $P(t, y), Q(t, y), q(t, y)$ и $p(t, y)$ являются 1-периодическими решениями начально-краевых задач на $Y_{0}$ :

$$
\begin{gathered}
m_{0} Q_{t}^{\prime}-\sqrt{\vartheta} \operatorname{div}_{y} A_{0}\left(\sqrt{\vartheta} \nabla_{y} Q+a_{0}^{0} Q\right)+\sqrt{\vartheta} k_{0}^{0} \nabla_{y} Q=0 \text { в } Y_{0} \times(0, \infty), \\
Q=0 \text { на } \bar{Y}_{1} \times(0, \infty),\left.\quad Q\right|_{t=0}=1 \quad \text { в } Y_{0}, \\
m_{0} P_{t}^{\prime}-\sqrt{\vartheta} \operatorname{div}_{y} A_{0}\left(\sqrt{\vartheta} \nabla_{y} P+a_{0}^{0} P\right)+\sqrt{\vartheta} k_{0}^{0} \nabla_{y} P=0 \text { в } Y_{0} \times(0, \infty), \\
P=0 \text { на } \bar{Y}_{1} \times(0, \infty),\left.\quad P\right|_{t=0}=\frac{r_{0}}{m_{0}} \text { в } Y_{0}, \\
m_{0} q_{t}^{\prime}-\sqrt{\vartheta} \operatorname{div}_{y} A_{0}\left(\sqrt{\vartheta} \nabla_{y} q+a_{0}^{0} q\right)+\sqrt{\vartheta} k_{0}^{0} \nabla_{y} q=0 \quad \text { в } Y_{0} \times(0, \infty), \\
q=0 \text { на } \bar{Y}_{1} \times(0, \infty),\left.\quad q\right|_{t=0}=\frac{\sqrt{\vartheta}\left(\operatorname{div}_{y} A_{0} a_{0}^{0}\right)}{m_{0}} \text { в } Y_{0}, \\
m_{0} p_{t}^{\prime}-\sqrt{\vartheta} \operatorname{div}_{y} A_{0}\left(\sqrt{\vartheta} \nabla_{y} p+a_{0}^{0} p\right)+\sqrt{\vartheta} k_{0}^{0} \nabla_{y} p=0 \quad \text { в } Y_{0} \times(0, \infty), \\
p=0 \text { на } \bar{Y}_{1} \times(0, \infty),\left.\quad p\right|_{t=0}=\frac{\sqrt{\vartheta}\left(\operatorname{div}_{y} A_{0} b_{0}^{0}\right)}{m_{0}} \text { в } Y_{0},
\end{gathered}
$$

где учтены обозначения (1.2), (1.6), (1.8) и (1.21). Далее введем обозначения

$$
\begin{array}{llll}
q_{0}=\left\langle m_{0} q\right\rangle_{\mathbf{0}}, & q_{k}=\sqrt{\vartheta}\left\langle k_{0}^{0} \nabla_{y} q\right\rangle_{\mathbf{0}}, & M_{k}=\left\langle m_{0} Q_{t}^{\prime}+\sqrt{\vartheta} k_{0}^{0} \nabla_{y} Q\right\rangle_{\mathbf{0}}, \\
p_{0}=\left\langle m_{0} p\right\rangle_{\mathbf{0}}, & p_{k}=\sqrt{\vartheta}\left\langle k_{0}^{0} \nabla_{y} p\right\rangle_{\mathbf{0}}, & R_{k}=\left\langle m_{0} P_{t}^{\prime}+\sqrt{\vartheta} k_{0}^{0} \nabla_{y} P\right\rangle_{\mathbf{0}}, \\
q_{\varepsilon}=q\left(t, \frac{x}{\varepsilon}\right), & p_{\varepsilon}=p\left(t, \frac{x}{\varepsilon}\right), & Q_{\varepsilon}=Q\left(t, \frac{x}{\varepsilon}\right), & P_{\varepsilon}=P\left(t, \frac{x}{\varepsilon}\right)
\end{array}
$$

и определим функцию $v$ как решение начально-краевой задачи для осредненного уравнения

$$
\begin{gathered}
M_{1} v_{t}^{\prime}-\left(M_{k}-q_{0}\right) *\left(v_{t}^{\prime}\right)-\operatorname{div} A(\nabla v+a v+b g)+K \nabla v+k_{a} v \\
+q_{k} * v+p_{0} *\left(g_{t}^{\prime}\right)+p_{k} * g+k_{b} g=R_{1} f-R_{k} * f \quad \text { в } \Omega \times(0, T), \\
v=0 \text { на } \partial \Omega \times(0, T),\left.\quad v\right|_{t=0}=w \text { в } \Omega,
\end{gathered}
$$

где использованы обозначения (1.13), (1.15) и (1.18).

Теорема 4. Пусть выполнены условия (1.2), (1.21), $f, g \in C_{0}^{\infty}((0, T) \times$ $\Omega) u w=0$. Предположим, что и-решение задачи (1.4) $и v-$-решение задачи (1.24). Тогда

$$
\begin{aligned}
& \max _{0 \leqslant t \leqslant T}\left\|u-v-P_{\varepsilon} * f+Q_{\varepsilon} *\left(v_{t}^{\prime}\right)-q_{\varepsilon} * v-p_{\varepsilon} * g\right\|_{L^{2}(\Omega)}^{2} \\
& \leqslant C\left(\varepsilon+\sqrt{\sigma}+\left|\vartheta-\frac{\sigma}{\varepsilon^{2}}\right|+\left|\sqrt{\vartheta}-\frac{\sqrt{\sigma}}{\varepsilon}\right|\right),
\end{aligned}
$$

где постоянная $C$ не зависит от $\varepsilon$ и $\sigma$.

Проверяется, что в условиях теоремы 4 достаточно гладкое решение задачи (1.24) существует и определено однозначно. Это утверждение доказывается в $\S 2$ методами работы [5], в которой была доказана разрешимость аналогичной задачи для линеаризованной системы уравнений гидродинамики. Отметим также, что используемые здесь задачи на ячейке (1.12), (1.14), (1.17), (1.22) имеют решения и не зависят от параметров $x, \varepsilon$ и $\sigma$. 
Доказательства представленных здесь утверждений даны в $\S 3-7$. Несколько вспомогательных лемм, используемых в этих доказательствах, приведены в $\S 2$. Частные случаи теорем 1-3 были анонсированы в докладе [13]. Утверждение, близкое к теореме 1 , было ранее доказано Н. С. Бахваловым.

Автор выражает признательность Н. С. Бахвалову за многочисленные полезные советы по рассматриваемым в статье вопросам.

\section{§2. Леммы}

В дальнейшем изложении $C$ обозначает постоянные, не зависящие от параметpов $\varepsilon$ и $\sigma$. Кроме того, для заданной 1-периодической на $Y$ функции $P(y)$ через $P_{\varepsilon}=P(x / \varepsilon)$ обозначается $\varepsilon$-периодическое продолжение этой функции на $\Omega$. Более точно, $P(y)$ продолжается как 1-периодическая функция на $\mathbb{R}^{n}$ и выбирается ограничение полученной функции при $y=x / \varepsilon$ на $\Omega$, которое и обозначается $P_{\varepsilon}$.

ЛЕмма 1. Для решений задачи (1.4) выполнено неравенство

$$
\begin{aligned}
& \max _{0 \leqslant t \leqslant T}\|u\|_{L^{2}(\Omega)}^{2}+\sigma\|\nabla u\|_{L^{2}\left(0, T ; L^{2}\left(\Omega_{0}^{\varepsilon}\right)^{n}\right)}^{2}+\|\nabla u\|_{L^{2}\left(0, T ; L^{2}\left(\Omega_{1}^{\varepsilon}\right)^{n}\right)}^{2} \\
& \quad \leqslant C\left(\|f\|_{L^{2}\left(0, T ; L^{2}(\Omega)\right)}^{2}+\|g\|_{L^{2}\left(0, T ; L^{2}(\Omega)\right)}^{2}+\|w\|_{L^{2}(\Omega)}^{2}\right) .
\end{aligned}
$$

ДоКАЗАТЕЛЬСТво. Выбирая $\varphi=u$ в тождестве (1.11) и учитывая (1.7), имеем

$$
\begin{aligned}
& \left(\frac{1}{2} \int_{\Omega} m_{\varepsilon} u^{2} d x\right)_{t}^{\prime}+\alpha \sigma \int_{\Omega_{0}^{\varepsilon}}(\nabla u)^{2} d x+\alpha \int_{\Omega_{1}^{\varepsilon}}(\nabla u)^{2} d x \\
& \quad \leqslant\left|\int_{\Omega}\left(k_{\varepsilon}^{\sigma} \nabla u\right) u d x\right|+\left|\int_{\Omega}\left(A_{\varepsilon}^{\sigma}\left(a_{\varepsilon} u+b_{\varepsilon} g\right), \nabla u\right) d x\right|+\left|\int_{\Omega} r_{\varepsilon} f u d x\right| .
\end{aligned}
$$

Воспользуемся для оценки интегралов в (2.1) неравенством Коши-Буняковского и " $\delta$-неравенством", записанным в виде $|a b| \leqslant(\delta / 2) a^{2}+b^{2} /(2 \delta)$ для положительного $\delta$ и произвольных $a, b$. Тогда

$$
\begin{aligned}
& \left|\int_{\Omega}\left(k_{\varepsilon}^{\sigma} \nabla u\right) u d x\right| \leqslant \sigma\left|\int_{\Omega_{0}^{\varepsilon}}\left(k_{0} \nabla u\right) u d x\right|+\left|\int_{\Omega_{1}^{\varepsilon}}\left(k_{1} \nabla u\right) u d x\right| \\
& \quad \leqslant \sigma \beta\|\nabla u\|_{L^{2}\left(\Omega_{0}^{\varepsilon}\right)^{n}}\|u\|_{L^{2}\left(\Omega_{0}^{\varepsilon}\right)}+\beta\|\nabla u\|_{L^{2}\left(\Omega_{1}^{\varepsilon}\right)^{n}}\|u\|_{L^{2}\left(\Omega_{1}^{\varepsilon}\right)} \\
& \quad \leqslant \beta\left(\frac{\sigma \delta}{2} \int_{\Omega_{0}^{\varepsilon}}(\nabla u)^{2} d x+\frac{\sigma}{2 \delta}\|u\|_{L^{2}\left(\Omega_{0}^{\varepsilon}\right)}^{2}+\frac{\delta}{2} \int_{\Omega_{1}^{\varepsilon}}(\nabla u)^{2} d x+\frac{1}{2 \delta}\|u\|_{L^{2}\left(\Omega_{1}^{\varepsilon}\right)}^{2}\right) .
\end{aligned}
$$

Следовательно, выбирая $\delta=\alpha / \beta$ в $(2.2)$ и используя это неравенство в $(2.1)$, интегралы с $\nabla u$ можно перенести в левую часть неравенства (2.1). Оценивая аналогично другие слагаемые справа в $(2.1)$, имеем

$$
\begin{aligned}
& \left(\int_{\Omega} m_{\varepsilon} u^{2} d x\right)_{t}^{\prime}+\sigma\|\nabla u\|_{L^{2}\left(\Omega_{0}^{\varepsilon}\right)^{n}}^{2}+\|\nabla u\|_{L^{2}\left(\Omega_{1}^{\varepsilon}\right)^{n}}^{2} \\
& \leqslant C\left(\|f\|_{L^{2}(\Omega)}^{2}+\|g\|_{L^{2}(\Omega)}^{2}+\|u\|_{L^{2}(\Omega)}^{2}\right) .
\end{aligned}
$$


Далее, учитывая (1.9) и применяя лемму Гронуолла (см., например, [1]), заключаем, что

$$
\begin{aligned}
& \|u(t)\|_{L^{2}(\Omega)}^{2}+\sigma \int_{0}^{t}\|\nabla u\|_{L^{2}\left(\Omega_{0}^{\varepsilon}\right)^{n}}^{2} d t+\int_{0}^{t}\|\nabla u\|_{L^{2}\left(\Omega_{1}^{\varepsilon}\right)^{n}}^{2} d t \\
& \leqslant C\left(\|w\|_{L^{2}(\Omega)}^{2}+\int_{0}^{t}\|f\|_{L^{2}(\Omega)}^{2} d t+\int_{0}^{t}\|g\|_{L^{2}(\Omega)}^{2} d t\right) .
\end{aligned}
$$

Как уже отмечалось, $u \in C^{0}\left([0, T] ; L^{2}(\Omega)\right)$. Таким образом, переход к максимуму по $t \in[0, T]$ в $(2.3)$ завершает доказательство леммы 1.

Будем обозначать через $H_{P}^{1}\left(Y_{1}\right)$ пространство Соболева 1-периодических функций из $H^{1}\left(Y_{1}\right)$ (см., например, [2], [3]). Эквивалентно, $H_{P}^{1}\left(Y_{1}\right)$ определяется как пространство квадратично интегрируемых вместе с производными первого порядка функций на многообразии $Y_{1}$ с границей $S$ класса $C^{2}$. Тогда [1] определены пространство $H_{P}^{1 / 2}(S)$ следов на $S$ элементов из $H_{P}^{1}\left(Y_{1}\right)$ и пространство $H_{P}^{-1 / 2}(S)$, сопряженное к $H_{P}^{1 / 2}(S)$. Кроме того, из результатов [14, гл. 1 , теорема 1.2] следует формула интегрирования по частям

$$
\int_{S}(V, \nu) W d s=\int_{Y_{1}}\left(\operatorname{div}_{y} V\right) W d y+\int_{Y_{1}}\left(V, \nabla_{y} W\right) d y
$$

где $W \in H_{P}^{1}\left(Y_{1}\right), \quad V \in L^{2}\left(Y_{1}\right)^{n}$ с $\operatorname{div}_{y} V \in L^{2}\left(Y_{1}\right)$ и след $(V, \nu)$ определен как элемент пространства $H_{P}^{-1 / 2}(S)$ :

$$
\|(V, \nu)\|_{H_{P}^{-1 / 2}(S)}^{2} \leqslant C\left(\|V\|_{L^{2}\left(Y_{1}\right)^{n}}^{2}+\left\|\operatorname{div}_{y} V\right\|_{L^{2}\left(Y_{1}\right)}^{2}\right) .
$$

Известно (см. [2], [3]), что решение $N_{1} \in\left(H_{P}^{1}\left(Y_{1}\right) \cap L^{2}\left(Y_{1}\right) / \mathbb{R}\right)^{n}$ задачи (1.12) сушествует и определено однозначно. Аналогично определены $N_{a}, N_{b} \in H_{P}^{1}\left(Y_{1}\right) \cap$ $L^{2}\left(Y_{1}\right) / \mathbb{R}$, удовлетворяюшие (1.14). Эти утверждения выполнены и при более слабых предположениях на $S$, но в дальнейшем встретятся более общие задачи Неймана на $Y_{1}$. Гладкость $S$ существенна в следующем известном (см., например, [1]) утверждении.

ЛЕмма 2. Пусть $V \in L^{2}\left(Y_{1}\right)$ u $W \in H_{P}^{-1 / 2}(S)$ удовлетворяют равенству

$$
\int_{Y_{1}} V d y=\int_{S} W d s
$$

Тогда существует единственное решение $U \in H_{P}^{1}\left(Y_{1}\right) \cap L^{2}\left(Y_{1}\right) / \mathbb{R}$ задачи Неймана на $Y_{1}$ :

$$
-\operatorname{div}_{y}\left(A_{1} \nabla_{y} U\right)=V \quad \text { в } \quad Y_{1}, \quad-\left(A_{1} \nabla_{y} U, \nu\right)=W \quad \text { на } \quad S .
$$

Доказательство следующей леммы повторяет доказательство леммы 1. 
ЛЕмма 3. Существуют однозначно определенные решения из пространства $L^{2}\left(0, T ; H_{P}^{1}(Y)\right) \cap L^{\infty}\left(0, T ; L^{2}(Y)\right)$ начально-краевых задач (1.17) и

$$
\begin{aligned}
& \|Q\|_{L^{\infty}\left(0, T ; L^{2}(Y)\right)}^{2}+\vartheta\left\|\nabla_{y} Q\right\|_{L^{2}\left(0, T ; L^{2}(Y)^{n}\right)}^{2} \leqslant C, \\
& \|P\|_{L^{\infty}\left(0, T ; L^{2}(Y)\right)}^{2}+\vartheta\left\|\nabla_{y} P\right\|_{L^{2}\left(0, T ; L^{2}(Y)^{n}\right)}^{2} \leqslant C,
\end{aligned}
$$

где постоянная $C$ не зависит от $\vartheta$.

Начальные данные в задачах (1.17) не удовлетворяют условиям согласования, и производные по $t$ от решений, используемые в (1.18), могут иметь особенности. Однако для дальнейшего достаточно следующего утверждения.

Лемма 4. Функции $M_{0}$ и $R_{0}$, определенные в $(1.18)$, принадлежат $L^{1}(0, T)$ $u$

$$
\left\|M_{0}\right\|_{L^{1}(0, T)}+\left\|R_{0}\right\|_{L^{1}(0, T)} \leqslant C
$$

где постоянная $C$ не зависит от $\vartheta$.

ДокаЗАТельСтво. Рассмотрим, например, первую начально-краевую задачу из (1.17). Для каждого $l=1,2, \ldots$ обозначим через $\lambda_{l}$ и $e_{l}$ собственные значения и собственные функции 1-периодической задачи Дирихле на $Y_{0}$ :

$$
-\operatorname{div}_{y}\left(A_{0} \nabla_{y} e_{l}\right)=\lambda_{l} m_{0} e_{l} \quad \text { в } Y_{0}, \quad e_{l}=0 \quad \text { на } \bar{Y}_{1}, \quad\left\langle m_{0} e_{l}^{2}\right\rangle_{\mathbf{0}}=1 .
$$

Более традиционно, здесь $e_{l}$ равны нулю на $S$ и продолжены нулем на $Y_{1}$. В соответствии с [4] имеем представление

$$
Q(y, t)=\sum_{l=1}^{\infty}\left\langle m_{0} e_{l}\right\rangle_{\mathbf{0}} e_{l}(y) e^{-\vartheta \lambda_{l} t}
$$

где ряд сходится в пространстве $L^{2}\left(0, T ; H_{P}^{1}\left(Y_{0}\right)\right)$. Далее, прямое вычисление дает неравенство

$$
\begin{aligned}
\left\|M_{0}\right\|_{L^{1}(0, T)} & =\int_{0}^{T}\left|\sum_{l=1}^{\infty}\left\langle m_{0} e_{l}\right\rangle_{\mathbf{0}}^{2}\left(-\vartheta \lambda_{l}\right) e^{-\vartheta \lambda_{l} t}\right| d t \\
& \leqslant \sum_{l=1}^{\infty}\left\langle m_{0} e_{l}\right\rangle_{\mathbf{0}}^{2} \int_{0}^{\infty} \vartheta \lambda_{l} e^{-\vartheta \lambda_{l} t} d t=\sum_{l=1}^{\infty}\left\langle m_{0} e_{l}\right\rangle_{\mathbf{0}}^{2}
\end{aligned}
$$

где сходимость последнего ряда эквивалентна принадлежности $\left.Q\right|_{t=0}$ пространству $L^{2}\left(Y_{0}\right)$, что доказывает лемму 4 .

Напомним, что свертка по $t$ от гладких функций $P(t, x)$ и $v(t, x)$ определяется равенствами

$$
(P * v)(t)=\int_{0}^{t} P(t-\tau) v(\tau) d \tau=\int_{0}^{t} P(\tau) v(t-\tau) d \tau
$$

и выполнены формулы дифференцирования

$$
(P * v)_{t}^{\prime}=\left(\left.P\right|_{t=0}\right) v+\left(P_{t}^{\prime}\right) * v=P\left(\left.v\right|_{t=0}\right)+P *\left(v_{t}^{\prime}\right) .
$$

Это определение можно продолжить достаточно стандартным образом на более общие классы функций и распределений (см., например, [15]). Некоторые из таких продолжений понадобятся в дальнейшем. 
Лемma 5. Пусть $P \in L^{2}\left(0, T ; L^{2}(\Omega)\right)$ u $v \in L^{2}\left(0, T ; L^{\infty}(\Omega)\right)$. Тогда $P * v \in$ $L^{\infty}\left(0, T ; L^{2}(\Omega)\right)$. Кроме того, $P * v \in C^{0}\left([0, T] ; L^{2}(\Omega)\right)$, если $v_{t}^{\prime} \in L^{2}\left(0, T ; L^{\infty}(\Omega)\right)$.

ДокАЗАТЕльСТво. Учитывая равенство

$$
\int_{0}^{t}\|P(t-\tau)\|_{L^{2}(\Omega)}^{2} d \tau=\int_{0}^{t}\|P(s)\|_{L^{2}(\Omega)}^{2} d s
$$

и неравенство Коши-Буняковского, получаем

$$
\begin{aligned}
& \|(P * v)(t)\|_{L^{2}(\Omega)} \leqslant \int_{0}^{t}\|P(t-\tau) v(\tau)\|_{L^{2}(\Omega)} d \tau \\
& \quad \leqslant \int_{0}^{t}\|P(t-\tau)\|_{L^{2}(\Omega)}\|v(\tau)\|_{L^{\infty}(\Omega)} d \tau \leqslant\|P\|_{L^{2}\left(0, T ; L^{2}(\Omega)\right)}\|v\|_{L^{2}\left(0, T ; L^{\infty}(\Omega)\right)},
\end{aligned}
$$

что доказывает первую часть леммы 5 . В соответствии со вторым равенством из $(2.7)$ имеем $(P * v)_{t}^{\prime} \in L^{2}\left(0, T ; L^{2}(\Omega)\right)$, если $v_{t}^{\prime} \in L^{2}\left(0, T ; L^{\infty}(\Omega)\right)$. Тогда лемма 5 следует из теоремы "вложения" (см., например, [14, гл. 3, лемма 1.2]).

Лемма 6. Пусть $P \in L^{1}(0, T) \quad u v \in L^{2}\left(0, T ; L^{2}(\Omega)\right)$. Тогдa $P * v \in$ $L^{2}\left(0, T ; L^{2}(\Omega)\right)$.

ДокаЗАТЕЛЬСтво. Выберем $h \in L^{2}\left(0, T ; L^{2}(\Omega)\right)$ с $\|h\|_{L^{2}\left(0, T ; L^{2}(\Omega)\right)}=1$ и продолжим $P, v, h$ нулем при $t \in \mathbb{R} \backslash[0, T]$. Тогда, используя теорему Фубини и инвариантность нормы в пространстве $L^{2}\left(\mathbb{R} ; L^{2}(\Omega)\right)$ относительно сдвигов по $t$, получаем

$$
\begin{aligned}
\left|\int_{-\infty}^{\infty} \int_{\Omega}(P * v)(t) h(t) d x d t\right| & \leqslant \int_{-\infty}^{\infty} \int_{\Omega} \int_{-\infty}^{t}|P(\tau)||v(t-\tau)||h(t)| d \tau d x d t \\
& \leqslant \int_{-\infty}^{\infty}|P(\tau)| \int_{\Omega} \int_{-\infty}^{\infty}|v(t-\tau)||h(t)| d t d x d \tau \\
& \leqslant \int_{0}^{T}|P(\tau)| d \tau\|v\|_{L^{2}\left(0, T ; L^{2}(\Omega)\right)}
\end{aligned}
$$

Супремум по $h$ от левой части этого неравенства задает норму в $L^{2}\left(0, T ; L^{2}(\Omega)\right)$, что доказывает лемму 6.

Лемма 7. Пусть иелое $s>0, \quad P \in L^{1}(0, T) u v \in L^{2}\left(0, T ; H^{s}(\Omega)\right)$. Тогда $P * v \in L^{2}\left(0, T ; H^{s}(\Omega)\right)$.

ДоКАЗАТЕЛЬСтво. Это утверждение следует из представления (2.6), леммы 6 и известных свойств пространств $H^{s}(\Omega)$ (см., например, [1]).

ЛЕмма 8. Пусть выполнены условия теоремы 2. Тогда существует единственное достаточно гладкое решение начально-краевой задачи (1.19), причем оценки соответствующих норм не зависят от $\vartheta$.

ДокАЗАТЕЛЬСТво. Обозначим $F=R_{1} f-R_{0} * f+\operatorname{div} A(b g)$. Из лемм 4 и 6 заключаем, что $F \in L^{2}\left(0, T ; L^{2}(\Omega)\right)$, причем оценка соответствующей нормы не зависит от $\vartheta$. Тогда из результатов работы [6] следует существование единственного $v \in H^{1}\left(0, T ; L^{2}(\Omega)\right) \cap L^{2}\left(0, T ; H_{0}^{1}(\Omega)\right)$, удовлетворяющего (1.19), с оценками соответствуюших норм, не зависяшими от $\vartheta$. 
Перепишем (1.19) в виде “эллиптической” краевой задачи:

$$
-\operatorname{div} A(\nabla v)=U \quad \text { в } \Omega \times(0, T), \quad v=0 \quad \text { на } \partial \Omega \times(0, T) .
$$

В силу эллиптической регулярности $v \in L^{2}\left(0, T ; H^{2}(\Omega)\right)$, поскольку $U \in$ $L^{2}\left(0, T ; L^{2}(\Omega)\right)$, с оценками, не зависяшими от $\vartheta$ (см., например, [1]).

Из лемм 4 и 7 следует, что $F \in L^{2}\left(0, T ; H^{s}(\Omega)\right)$. Здесь и далее целое положительное $s$ достаточно велико и соответствующие оценки не зависят от $\vartheta$. Учитывая второе равенство из (2.7) и финитность $f, g$ вблизи $t=0$, имеем $F \in H^{s}\left(0, T ; H^{s}(\Omega)\right)$. Полагая $t=0$ в первом уравнении из (1.19), получаем $\left.v_{t}^{\prime}\right|_{t=0}=0$, поскольку $w=0$. Вновь учитывая второе равенство из $(2.7)$, продифференцируем первое уравнение из (1.19) по $t$. Тогда $v_{t}^{\prime}$ удовлетворяет начально-краевой задаче, аналогичной исходной. Следовательно, $v_{t}^{\prime} \in H^{1}\left(0, T ; L^{2}(\Omega)\right) \cap$ $L^{2}\left(0, T ; H^{2}(\Omega)\right)$, и поэтому $v \in H^{2}\left(0, T ; H^{2}(\Omega)\right) \cap L^{2}\left(0, T ; H^{4}(\Omega)\right)$ в силу леммы 7 и представления (2.8) (см., например, [1]). Продолжая по индукции, получаем $v \in H^{s}\left(0, T ; H^{s}(\Omega)\right)$, что доказывает лемму 8 .

На самом деле в $\S 3-6$ используется только гладкость конечного порядка решений осредненных задач (1.16), (1.19) и (1.20):

$$
\partial_{t}^{\varkappa} \partial_{x_{1}}^{\varkappa_{1}} \ldots \partial_{x_{n}}^{\varkappa_{n}} v \in L^{2}\left(0, T ; L^{\infty}(\Omega)\right) \text { для } \varkappa+\varkappa_{1}+\ldots+\varkappa_{n} \leqslant 3,
$$

где через $\partial_{t}, \partial_{x_{1}}, \ldots, \partial_{x_{n}}$ обозначены соответствуюшие частные производные. Достаточные для выполнения (2.9) условия на исходные данные для задач (1.16) и (1.20) можно найти в [4], а для задачи (1.19) выводятся из доказательства леммы 8 и теорем вложений. Но мы не будем останавливаться на формулировке этих громоздких предположений на $f, g, w$ и $\Omega$.

Рассмотрим теперь задачу (1.4) в предположении, что выполнены равенства (1.21). Отметим, что лемма 1 выполнена и для решений этой задачи. Действительно, чтобы доказать это утверждение, достаточно оценить подходяшим образом дополнительные слагаемые, возникаюшие в (2.1). Но, как и в доказательстве (2.2), получаем

$$
\begin{aligned}
\sqrt{\sigma}\left|\int_{\Omega_{0}^{\varepsilon}}\left(A_{0} a_{0}^{0} u, \nabla u\right) d x\right| & \leqslant \sqrt{\sigma} C\|u\|_{L^{2}\left(\Omega_{0}^{\varepsilon}\right)}\|\nabla u\|_{L^{2}\left(\Omega_{0}^{\varepsilon}\right)^{n}} \\
& \leqslant C\left(\frac{\sqrt{\sigma} \delta}{2} \int_{\Omega_{0}^{\varepsilon}}(\nabla u)^{2} d x+\frac{\sqrt{\sigma}}{2 \delta}\|u\|_{L^{2}\left(\Omega_{0}^{\varepsilon}\right)}^{2}\right), \\
\sqrt{\sigma}\left|\int_{\Omega_{0}^{\varepsilon}}\left(k_{0}^{0} \nabla u\right) u d x\right| & \leqslant C\left(\frac{\sqrt{\sigma} \delta}{2} \int_{\Omega_{0}^{\varepsilon}}(\nabla u)^{2} d x+\frac{\sqrt{\sigma}}{2 \delta}\|u\|_{L^{2}\left(\Omega_{0}^{\varepsilon}\right)}^{2}\right) .
\end{aligned}
$$

Следовательно, выбирая $\delta=\alpha \sqrt{\sigma} /(2 C)$ в (2.10) и используя эти неравенства в (2.1), интегралы с $\nabla u$ перенесем в левую часть неравенства (2.1). Далее, как и в доказательстве леммы 1 , получим оценку $(2.3)$, из которой и следует лемма 1 .

Отметим также, что лемма 3 выполнена и для решений начально-краевых задач (1.22), но с постоянными $C$, зависящими от $\vartheta$, впрочем несложно выписать явно эту зависимость (см., например, [4]). В доказательстве леммы 4 используется то, что производящий оператор соответствующей начально-краевой задачи самосопряжен. Для задач (1.22) это условие может не вьполняться. Однако лемма 4 используется только при доказательстве сушествования достаточно гладкого 
решения задачи (1.19), а для задачи (1.24) это утверждение будет доказано непосредственно с помощью преобразования Лапласа.

Фиксируем $\gamma \geqslant 0$ и определим пространство $L_{\gamma}^{2}\left(0, \infty ; L^{2}(\Omega)\right)$ как множество функций из $L_{\mathrm{loc}}^{2}\left(0, \infty ; L^{2}(\Omega)\right)$, для которых конечна величина

$$
\|u\|_{L_{\gamma}^{2}\left(0, \infty ; L^{2}(\Omega)\right)}=\left\|e^{-\gamma t} u\right\|_{L^{2}\left(0, \infty ; L^{2}(\Omega)\right)} .
$$

Последнее равенство определяет норму в пространстве $L_{\gamma}^{2}\left(0, \infty ; L^{2}(\Omega)\right)$, относительно которой это пространство является полным (см., например, [16]).

Следуя [16], определим пространство $E_{\gamma}\left(L^{2}(\Omega)\right)$ как множество функций $U(\rho)=$ $U\left(\rho_{1}+i \rho_{2}\right)$ со значениями в $L^{2}(\Omega)$, заданных и голоморфных в комплексной полуплоскости $\mathbb{C}_{\gamma}=\left\{\rho \in \mathbb{C}: \rho=\rho_{1}+i \rho_{2}, \rho_{1}>\gamma\right\}$, для которых конечна величина

$$
\|U(\rho)\|_{E_{\gamma}\left(L^{2}(\Omega)\right)}^{2}=\int_{-\infty}^{\infty}\left\|U\left(\gamma+i \rho_{2}\right)\right\|_{L^{2}(\Omega)}^{2} d \rho_{2},
$$

где интеграл определяется как предел в среднем интеграла от $K$ до $-K$ при $K \rightarrow \infty$. Последнее равенство определяет норму в пространстве $E_{\gamma}\left(L^{2}(\Omega)\right)$. Следуюший вариант теоремы Пэли-Винера доказан в [16].

ТЕОРема 9. Преобразование Лапласа

$$
\widehat{u(t)}=\int_{0}^{\infty} e^{-\rho t} u(t) d t=U(\rho)
$$

отображсает пространство $L_{\gamma}^{2}\left(0, \infty ; L^{2}(\Omega)\right)$ на пространство $E_{\gamma}\left(L^{2}(\Omega)\right)$ взаимно однозначно и взаимно непрерывно.

Аналогично определяются пространства $E_{\gamma}\left(H_{0}^{1}(\Omega)\right)$ и $E_{\gamma}\left(L^{2}(Y)\right)$, для которых также выполнен аналог теоремы 9. Кроме того (см. [16]), преобразование Лапласа коммутирует с дифференцированиями по пространственным переменным и оператор свертки по $t$ переводится преобразованием Лапласа в оператор поточечного умножения.

Лемма 10. Пусть выполнены условия теоремы 4. Тогда существует единственное достаточно гладкое решение начально-краевой задачи (1.24).

ДокАЗАТЕльство. Для доказательства существования и единственности решения в подходящих пространствах, как обычно, наиболее существенный момент это априорные оценки (см., например, [1], [4]). В соответствии с теоремой 9 эти оценки можно попытаться доказать для преобразования Лапласа от решения задачи (1.24). Введем обозначения $V=\widehat{v}, F=\widehat{f}, G=\widehat{g}$ для преобразований Лапласа от функций $v, f$ и $g$ из (1.24). Тогда, применяя преобразование Лапласа к (1.24), получаем краевую задачу для $V$ с комплексным параметром $\rho$ :

$$
\rho m V-\left(\rho m_{k}-q_{0 k}\right) V+2 \mathbb{A} V=\mathbb{F} \quad \text { в } \Omega, \quad V=0 \text { на } \partial \Omega
$$

(см., например, [16], [17]), где использованы обозначения

$$
\begin{aligned}
m & =M_{1}, \quad 2 \mathbb{A} V=-\operatorname{div} A(\nabla V+a V)+K \nabla V+k_{a} V, \\
\mathbb{F} & =R_{1} F-\widehat{R}_{k} F-\left(\rho \widehat{p}_{0}+\widehat{p}_{k}\right) G-k_{b} G+A b \nabla G, \\
m_{k} & =\widehat{M}_{k}=\left\langle m_{0} \rho \widehat{Q}-\left.m_{0}(Q)\right|_{t=0}+\sqrt{\vartheta} k_{0}^{0} \nabla_{y} \widehat{Q}\right\rangle_{\mathbf{0}} \\
& =\left\langle m_{0}(\rho \widehat{Q}-1)+\sqrt{\vartheta} k_{0}^{0} \nabla_{y} \widehat{Q}\right\rangle_{\mathbf{0}}, \\
q_{0 k} & =\left\langle m_{0} \rho \widehat{q}+\sqrt{\vartheta} k_{0}^{0} \nabla_{y} \widehat{q}\right\rangle_{\mathbf{0}}, \\
\widehat{R}_{k} & =\left\langle m_{0} \rho \widehat{P}-r_{0}+\sqrt{\vartheta} k_{0}^{0} \nabla_{y} \widehat{P}\right\rangle_{\mathbf{0}} .
\end{aligned}
$$


Отметим, что в силу леммы 3 функции $\widehat{Q}(\rho), \widehat{P}(\rho), \widehat{q}(\rho), \widehat{p}(\rho)$, и поэтому $m_{k}(\rho)$, $q_{0 k}(\rho), \widehat{R}_{k}(\rho), \widehat{p}_{0}(\rho), \widehat{p}_{k}(\rho)$ определены и являются голоморфными функциями на $\mathbb{C}_{0}$ со значениями в соответствуюших пространствах (см., например, $[17$, гл. 6$]$ ). Из (1.22) получаем для $z=\widehat{P}(\rho)$ краевую задачу

$$
\begin{gathered}
m_{0} \rho z-\sqrt{\vartheta} \operatorname{div}_{y} A_{0}\left(\sqrt{\vartheta} \nabla_{y} z+a_{0}^{0} z\right)+\sqrt{\vartheta} k_{0}^{0} \nabla_{y} z=r_{0} \quad \text { в } Y_{0}, \\
z=0 \text { на } \bar{Y}_{1} .
\end{gathered}
$$

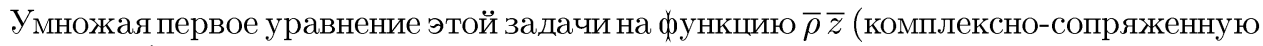
к $\rho z=\rho \widehat{P}(\rho))$ и интегрируя полученное соотношение по $Y_{0}$, имеем

$$
\begin{aligned}
& \left\langle m_{0}(\rho z)^{2}\right\rangle_{\mathbf{0}}+\bar{\rho} \vartheta\left\langle\left(A_{0} \nabla_{y} z, \nabla_{y} \bar{z}\right)\right\rangle_{\mathbf{0}} \\
& \quad=\left\langle r_{0} \bar{\rho} \bar{z}\right\rangle_{\mathbf{0}}-\left\langle\left(a_{0}^{0} \bar{\rho} z, \sqrt{\vartheta} \nabla_{y} \bar{z}\right)\right\rangle_{\mathbf{0}}-\left\langle\bar{\rho} \bar{z} \sqrt{\vartheta} k_{0}^{0} \nabla_{y} z\right\rangle_{\mathbf{0}} .
\end{aligned}
$$

Далее рассмотрим уравнение, комплексно-сопряженное к первому уравнению из (2.13). Умножая это уравнение на $\rho z$ и интегрируя по $Y_{0}$, получаем

$$
\begin{aligned}
& \left\langle m_{0}(\rho z)^{2}\right\rangle_{\mathbf{0}}+\rho \vartheta\left\langle\left(A_{0} \nabla_{y} \bar{z}, \nabla_{y} z\right)\right\rangle_{\mathbf{0}} \\
& \quad=\left\langle r_{0} \rho z\right\rangle_{\mathbf{0}}-\left\langle\left(a_{0}^{0} \rho \bar{z}, \sqrt{\vartheta} \nabla_{y} z\right)\right\rangle_{\mathbf{0}}-\left\langle\rho z \sqrt{\vartheta} k_{0}^{0} \nabla_{y} \bar{z}\right\rangle_{\mathbf{0}} .
\end{aligned}
$$

Учитьвая симметричность матрищы $A_{0}$, сложим соотношения (2.14) и (2.15). Тогда

$$
\begin{aligned}
& \left\langle m_{0}(\rho z)^{2}\right\rangle_{\mathbf{0}}+\rho_{1} \vartheta\left\langle\left(A_{0} \nabla_{y} z, \nabla_{y} \bar{z}\right)\right\rangle_{\mathbf{0}} \\
& \quad=\operatorname{Re}\left\langle r_{0} \bar{\rho} \bar{z}\right\rangle_{\mathbf{0}}-\operatorname{Re}\left\langle\left(a_{0}^{0} \bar{\rho} z, \sqrt{\vartheta} \nabla_{y} \bar{z}\right)\right\rangle_{\mathbf{0}}-\operatorname{Re}\left\langle\bar{\rho} \bar{z} \sqrt{\vartheta} k_{0}^{0} \nabla_{y} z\right\rangle_{\mathbf{0}},
\end{aligned}
$$

где по определению $\operatorname{Re} z=(z+\bar{z}) / 2$, и поэтому $|\operatorname{Re} z| \leqslant(|z|+|\bar{z}|) / 2=|z|$ для $z \in \mathbb{C}$. Следовательно, найдутся такие постоянные $c_{0}$ и $C$, не зависяшие от $\vartheta$ и $\rho \in \mathbb{C}_{0}$, что

$\|\rho z\|_{L^{2}\left(Y_{0}\right)}^{2}+\rho_{1} \vartheta\left\|\nabla_{y} z\right\|_{L^{2}\left(Y_{0}\right)^{n}}^{2} \leqslant \frac{1}{2}\|\rho z\|_{L^{2}\left(Y_{0}\right)}^{2}+c_{0} \vartheta\left\|\nabla_{y} z\right\|_{L^{2}\left(Y_{0}\right)^{n}}^{2}+C\left\|r_{0}\right\|_{L^{2}\left(Y_{0}\right)}^{2}$

(см. доказательство леммы 1 ). Таким образом, для всех $\rho=\rho_{1}+i \rho_{2}$ с $\rho_{1}-c_{0} \geqslant 1$ выполнено неравенство

$$
\frac{1}{2}\|\rho z\|_{L^{2}\left(Y_{0}\right)}^{2}+\vartheta\left\|\nabla_{y} z\right\|_{L^{2}\left(Y_{0}\right)^{n}}^{2} \leqslant C\left\|r_{0}\right\|_{L^{2}\left(Y_{0}\right)}^{2} .
$$

Аналогичные неравенства выполнены для функций $\widehat{Q}(\rho), \widehat{q}(\rho)$ и $\widehat{p}(\rho)$. Поэтому найдется такое положительное $\omega=1+c_{0}$, что

$$
\begin{aligned}
& \left|q_{0 k}\right| \leqslant\left|\left\langle m_{0} \rho \widehat{q}\right\rangle_{\mathbf{0}}\right|+\left|\left\langle\sqrt{\vartheta} k_{0}^{0} \nabla_{y} \widehat{q}\right\rangle_{\mathbf{0}}\right| \leqslant C, \\
& \left|m_{k}\right| \leqslant C, \quad\left|\rho \widehat{p}_{0}+\widehat{p}_{k}\right| \leqslant C, \quad\left|\widehat{R}_{k}\right| \leqslant C,
\end{aligned}
$$

где использованы обозначения (2.12) и постоянная $C$ не зависит от $\vartheta$ и $\rho \in \mathbb{C}_{\omega}$. Следовательно, вьполнено неравенство

$$
\|\mathbb{F}\|_{L^{2}(\Omega)} \leqslant C\left(\|F\|_{L^{2}(\Omega)}+\|G\|_{H^{1}(\Omega)}\right),
$$

где постоянная $C$ не зависит от $\vartheta$ и $\rho \in \mathbb{C}_{\omega}$. 
Умножим уравнение из (2.11) на $\bar{V}$ и проинтегрируем полученное соотношение по $\Omega$. Тогда

$$
\rho m \int_{\Omega} V^{2} d x+\int_{\Omega}\left(q_{0 k}-\rho m_{k}\right) V^{2} d x+2 \int_{\Omega}(\mathbb{A} V) \bar{V} d x=\int_{\Omega} \mathbb{F} \bar{V} d x .
$$

Далее рассмотрим уравнение, комплексно-сопряженное к уравнению из (2.11). УМножая это уравнение на $V$, интегрируя по $\Omega$ и складывая полученное соотношение с (2.19), имеем

$$
\begin{aligned}
& \rho_{1} m \int_{\Omega} V^{2} d x+\int_{\Omega} \operatorname{Re}\left(q_{0 k}-\rho m_{k}\right) V^{2} d x \\
& \quad+\int_{\Omega}(\mathbb{A} V) \bar{V} d x+\int_{\Omega}(\mathbb{A} \bar{V}) V d x=\operatorname{Re} \int_{\Omega} \mathbb{F} \bar{V} d x .
\end{aligned}
$$

Оценим интегралы в этом равенстве, содержашие $\mathbb{A}$, как и в доказательстве леммы 1 (сохраняя $(A \nabla V, \nabla \bar{V})$ в левой части $(2.20)$ и перенося остальные слагаемые в правую часть (2.20)). Тогда найдутся такие постоянные $c_{1}, c_{\alpha}$ и $C$, не зависящие от $\vartheta$ и $\rho \in \mathbb{C}_{\omega}$, что

$$
\begin{gathered}
\rho_{1} m\|V\|_{L^{2}(\Omega)}^{2}+\int_{\Omega} \operatorname{Re}\left(-\rho m_{k}\right) V^{2} d x+c_{\alpha}\|\nabla V\|_{L^{2}(\Omega)^{n}}^{2} \\
\leqslant C\|\mathbb{F}\|_{L^{2}(\Omega)}^{2}+c_{1}\|V\|_{L^{2}(\Omega)}^{2}+\frac{c_{\alpha}}{2}\|\nabla V\|_{L^{2}(\Omega)^{n}}^{2},
\end{gathered}
$$

где учтены неравенства (2.17) и положительная определенность матрищы $A$. Предположим, что выполнено соотношение

$$
\operatorname{Re}\left(-\rho m_{k}\right)=\mathbb{P}+\mathbb{B} \text { при } \mathbb{P} \geqslant 0 \text { и }|\mathbb{B}| \leqslant c_{2}
$$

с некоторой постоянной $c_{2}$, не зависящей от $\rho \in \mathbb{C}_{\omega}$. Тогда из $(2.21)$ следует неравенство

$$
\left(\rho_{1} m-c_{1}-c_{2}\right)\|V\|_{L^{2}(\Omega)}^{2}+\frac{c_{\alpha}}{2}\|\nabla V\|_{L^{2}(\Omega)^{n}}^{2} \leqslant C\|\mathbb{F}\|_{L^{2}(\Omega)}^{2} .
$$

Таким образом, для всех $\rho=\rho_{1}+i \rho_{2}\left(\right.$ с $\rho_{1}-c_{1}-c_{2} \geqslant 1$ и $\left.\rho_{1}-c_{0} \geqslant 1\right)$ найдется такое положительное $\omega$, что

$$
\|V\|_{H^{1}(\Omega)} \leqslant C\|\mathbb{F}\|_{L^{2}(\Omega)} \text { при } \rho_{1} \geqslant \omega
$$

где постоянная $C$ не зависит от $\rho \in \mathbb{C}_{\omega}$.

Докажем соотношения (2.22). Пусть $z=\widehat{Q}(\rho)$. Тогда

$$
-\rho m_{k}=\rho\left\langle(-1) m_{0}(\rho z-1)\right\rangle_{\mathbf{0}}-\sqrt{\vartheta}\left\langle k_{0}^{0} \nabla_{y} \rho z\right\rangle_{\mathbf{0}} .
$$

Интегрируя в последнем слагаемом этого равенства по частям, имеем

$$
-\sqrt{\vartheta}\left\langle k_{0}^{0} \nabla_{y} \rho z\right\rangle_{\mathbf{0}}=\sqrt{\vartheta}\left\langle\left(\operatorname{div}_{y} k_{0}^{0}\right) \rho z\right\rangle_{\mathbf{0}} .
$$

Поэтому, учитывая предположение $\operatorname{div}_{y} k_{0}^{0} \in L^{\infty}\left(Y_{0}\right)$ и неравенство $(2.16)$, получаем

$$
\left|\operatorname{Re} \sqrt{\vartheta}\left\langle k_{0}^{0} \nabla_{y} \rho z\right\rangle_{\mathbf{0}}\right| \leqslant \sqrt{\vartheta}\left|\left\langle\left(\operatorname{div}_{y} k_{0}^{0}\right) \rho z\right\rangle_{\mathbf{0}}\right| \leqslant C
$$

7

Серия математическая, №5 
где постоянная $C$ не зависит от $\rho \in \mathbb{C}_{\omega}$. Следовательно, последнее слагаемое в (2.24) можно считать нулевым, поскольку это слагаемое не влияет на доказательство соотношений (2.22).

В соответствии с $(1.22)$ краевая задача для $z=\widehat{Q}(\rho)$ имеет вид

$$
\begin{gathered}
m_{0}(\rho z-1)-\sqrt{\vartheta} \operatorname{div}_{y} A_{0}\left(\sqrt{\vartheta} \nabla_{y} z+a_{0}^{0} z\right)+\sqrt{\vartheta} k_{0}^{0} \nabla_{y} z=0 \text { в } Y_{0}, \\
z=0 \text { на } \bar{Y}_{1} .
\end{gathered}
$$

Умножая уравнение этой задачи на функцию $\rho \bar{\rho} \bar{z}$ и интегрируя полученное соотношение по $Y_{0}$, имеем

$$
\begin{aligned}
& \rho\left\langle m_{0} \bar{\rho} \bar{z}(\rho z-1)\right\rangle_{\mathbf{0}}+\vartheta\left\langle\left(A_{0} \nabla_{y} \rho z, \nabla_{y} \bar{\rho} \bar{z}\right)\right\rangle_{\mathbf{0}} \\
& \left.\quad+\left\langle\left(A_{0} a_{0}^{0} \rho z, \sqrt{\vartheta} \nabla_{y} \bar{\rho} \bar{z}\right)\right\rangle_{\mathbf{0}}+\left\langle\left(\sqrt{\vartheta} k_{0}^{0} \nabla_{y} \rho z\right) \bar{\rho} \bar{z}\right)\right\rangle_{\mathbf{0}}=0 .
\end{aligned}
$$

Учтем это соотношение в равенстве (2.24). Тогда

$$
\begin{aligned}
-\rho m_{k}= & \rho\left\langle m_{0}(\rho z-1)^{2}\right\rangle_{\mathbf{0}}+\vartheta\left\langle\left(A_{0} \nabla_{y} \rho z, \nabla_{y} \bar{\rho} \bar{z}\right)\right\rangle_{\mathbf{0}} \\
& \left.+\left\langle\left(A_{0} a_{0}^{0} \rho z, \sqrt{\vartheta} \nabla_{y} \bar{\rho} \bar{z}\right)\right\rangle_{\mathbf{0}}+\left\langle\left(\sqrt{\vartheta} k_{0}^{0} \nabla_{y} \rho z\right) \bar{\rho} \bar{z}\right)\right\rangle_{\mathbf{0}}
\end{aligned}
$$

и аналогичное равенство выполнено для $-\bar{\rho} \bar{m}_{k}$. Интегрируя в последнем слагаемом равенства (2.27) по частям, получаем

$$
\left\langle\left(\sqrt{\vartheta} k_{0}^{0} \nabla_{y} \rho z\right) \bar{\rho} \bar{z}\right\rangle_{\mathbf{0}}=-\left\langle\rho z \sqrt{\vartheta} k_{0}^{0} \nabla_{y} \bar{\rho} \bar{z}\right\rangle_{\mathbf{0}}-\sqrt{\vartheta}\left\langle(\rho z)^{2} \operatorname{div} k_{0}^{0}\right\rangle_{\mathbf{0}}
$$

Последнее слагаемое в этом равенстве можно считать нулевым в силу неравенства, аналогичного (2.25), а предпоследнее слагаемое сократится с соответствующим слагаемым из $-\bar{\rho} \bar{m}_{k}$ при вычислении $\operatorname{Re}\left(-\bar{\rho} \bar{m}_{k}\right)$. Следовательно, преобразовывая аналогичным образом предпоследнее слагаемое в $(2.27)$, имеем

$$
\operatorname{Re}\left(-\rho m_{k}\right)=-\frac{\rho m_{k}+\bar{\rho} \bar{m}_{k}}{2}=\rho_{1}\left\langle m_{0}(\rho z-1)^{2}\right\rangle_{\mathbf{0}}+\vartheta\left\langle A_{0} \nabla_{y} \rho z, \nabla_{y} \bar{\rho} \bar{z}\right\rangle_{\mathbf{0}} \geqslant 0
$$

с точностью до несушественных слагаемых, что доказывает соотношения (2.22) и неравенство (2.23).

Из неравенств $(2.18)$ и $(2.23)$ следует, что для каждого $\rho \in \mathbb{C}_{\omega}$ решение задачи (2.11) существует и определено однозначно, поскольку уравнение этой задачи является обычным эллиптическим уравнением с комплексными коэффициентами (см., например, $[1],[16])$. Это решение является непрерьвной функцией на $\mathbb{C}_{\omega}$ со значениями в $H_{0}^{1}(\Omega)$. Действительно, фиксируем несовпадающие $\rho$ и $\rho_{0}$ из $\mathbb{C}_{\omega}$. Тогда $V_{0}=V(\rho)-V\left(\rho_{0}\right)$ является решением краевой задачи

$$
\begin{aligned}
& \rho m V_{0}-\left(\rho m_{k}-q_{0 k}\right) V_{0}+2 \mathbb{A} V_{0}=\mathbb{F}(\rho)-\mathbb{F}\left(\rho_{0}\right) \\
& \quad+\left(\mathbb{M}(\rho)-\mathbb{M}\left(\rho_{0}\right)\right) V\left(\rho_{0}\right) \text { в } \Omega, \quad V_{0}=0 \text { на } \partial \Omega,
\end{aligned}
$$

где $\mathbb{F}$ и $\mathbb{M}=\rho m-\rho m_{k}+q_{0 k}$ являются голоморфными (и поэтому непрерывными) функциями на $\mathbb{C}_{0}$ со значениями в соответствуюших пространствах. Следовательно, используя неравенство (2.23), имеем

$$
\left\|V(\rho)-V\left(\rho_{0}\right)\right\|_{H_{0}^{1}(\Omega)} \rightarrow 0 \quad \text { при } \rho \rightarrow \rho_{0}
$$

что доказывает непрерывность решения задачи (2.11). 
Продифференцируем формально (2.11) по $\rho$ и рассмотрим краевую задачу

$$
\rho m V_{1}-\left(\rho m_{k}-q_{0 k}\right) V_{1}+2 \mathbb{A} V_{1}=\mathbb{F}_{\rho}^{\prime}+\mathbb{M}_{\rho}^{\prime} V \quad \text { в } \Omega, \quad V_{1}=0 \quad \text { на } \partial \Omega .
$$

В силу уже доказанных результатов решение этой задачи сушествует, непрерывно зависит от $\rho \in \mathbb{C}_{\omega}$ и удовлетворяет неравенству, аналогичному (2.23).

Разделим уравнения $(2.29)$ на $\rho-\rho_{0}$ и вычтем из полученных соотношений уравнения (2.31). Тогда $V_{2}=\left(V(\rho)-V\left(\rho_{0}\right)\right) /\left(\rho-\rho_{0}\right)-V_{1}$ является решением краевой задачи

$$
\begin{aligned}
\rho m V_{2}-\left(\rho m_{k}-q_{0 k}\right) V_{2}+2 \mathbb{A} V_{2} & =\mathbb{F}_{\Delta}-\mathbb{F}_{\rho}^{\prime}+\mathbb{M}_{\Delta} V_{0}+\left(\mathbb{M}_{\Delta}-\mathbb{M}_{\rho}^{\prime}\right) V \text { в } \Omega, \\
V_{2} & =0 \text { на } \partial \Omega
\end{aligned}
$$

где $\mathbb{F}_{\Delta}=\left(\mathbb{F}(\rho)-\mathbb{F}\left(\rho_{0}\right)\right) /\left(\rho-\rho_{0}\right)$ и $\mathbb{M}_{\Delta}=\left(\mathbb{M}(\rho)-\mathbb{M}\left(\rho_{0}\right)\right) /\left(\rho-\rho_{0}\right)$. Следовательно, используя неравенство $(2.23)$ и соотношение $(2.30)$, имеем

$$
\left\|\left(V(\rho)-V\left(\rho_{0}\right)\right) /\left(\rho-\rho_{0}\right)-V_{1}\right\|_{H_{0}^{1}(\Omega)} \rightarrow 0 \text { при } \rho \rightarrow \rho_{0} .
$$

Таким образом, решение задачи (2.11) является голоморфной функцией на $\mathbb{C}_{\omega}$ со значениями в $H_{0}^{1}(\Omega)$.

Кроме того, умножая неравенство $(2.23)$ на $|\rho|^{s}$ для $s=0,1, \ldots$, имеем априорные оценки

$$
\left\|\rho^{s} V\right\|_{H^{1}(\Omega)} \leqslant C\left(\left\|\rho^{s} F\right\|_{L^{2}(\Omega)}+\left\|\rho^{s} G\right\|_{H^{1}(\Omega)}\right),
$$

где постоянная $C$ не зависит от $s$ и $\rho \in \mathbb{C}_{\omega}$. Из этих оценок и теоремы 9 следует, что

$$
\left\|v_{t}^{(s)}\right\|_{L_{\omega}^{2}\left(0, \infty ; H^{1}(\Omega)\right)} \leqslant C\left(\left\|f_{t}^{(s)}\right\|_{L_{\omega}^{2}\left(0, \infty ; L^{2}(\Omega)\right)}^{2}+\left\|g_{t}^{(s)}\right\|_{L_{\omega}^{2}\left(0, \infty ; H^{1}(\Omega)\right)}^{2}\right),
$$

где постоянная $C$ не зависит от $s=0,1, \ldots$ Весовая функция $e^{-\omega t}$ из определения пространства $L_{\omega}^{2}\left(0, \infty ; L^{2}(\Omega)\right)$ отделена от нуля на конечном интервале $(0, T)$, и поэтому

$$
\|v\|_{H^{s}\left(0, T ; H^{1}(\Omega)\right)} \leqslant C\left(\|f\|_{H^{s}\left(0, T ; L^{2}(\Omega)\right)}+\|g\|_{H^{s}\left(0, T ; H^{1}(\Omega)\right)}\right),
$$

где постоянная $C$ не зависит от $s=0,1, \ldots$, но зависит от $T$.

В условиях леммы из неравенств (2.32) следует, что $\left.v_{t}^{(s)}\right|_{t=0}=0$ для $s=0,1, \ldots$ (см., например, [16]). Эти равенства можно получить и непосредственно из (1.24). Действительно, полагая $t=0$ в этих уравнениях, получаем $\left.v_{t}^{\prime}\right|_{t=0}=0$. Учитывая второе равенство из (2.7), продифференцируем уравнения (1.24) по $t$ и положим $t=0$. Тогда $\left.v_{t}^{(2)}\right|_{t=0}=0$, и, продолжая по индукции, получим требуемые равенства.

Отметим, что приведенное доказательство неравенства (2.33) не обобщается на случай ненулевых $w$. Действительно, пусть выполнены неравенства (2.33) и $\left.v\right|_{t=0} \neq 0$. Тогда, в общем случае, $\left.v_{t}^{\prime}\right|_{t=0} \neq 0$ и дифференцирование уравнений (1.24) по $t$ приводит к появлению в этих уравнениях нерегулярного по $t$ слагаемого $M_{k}(t)\left(\left.v_{t}^{\prime}\right|_{t=0}\right)$, а остальные слагаемые регулярны по $t$, что несовместимо с достаточной гладкостью решения задачи (1.24). Нерегулярность $M_{k}(t)$ связана 
с возможной нерегулярностью по $t$ решений задач $(1.22)$, для которых не выполнены условия согласования подходящего порядка (см., например, [1], [4], [16]).

Перепишем (1.24) в виде "эллиптической" краевой задачи:

$$
-\operatorname{div}(A \nabla v)=U \quad \text { в } \Omega \times(0, T), \quad v=0 \quad \text { на } \quad \partial \Omega \times(0, T) .
$$

Из теоремы 9, неравенств (2.17) и (2.32) следует, что $U \in H^{s-1}\left(0, T ; L^{2}(\Omega)\right)$, где целое положительное $s$ достаточно велико. Отметим, что $\left|\rho m_{k}\right| \leqslant|\rho|\left|m_{k}\right| \leqslant|\rho| C$ в соответствии с неравенствами (2.17). Поэтому для решения задачи (2.34) имеем $v \in H^{s-1}\left(0, T ; H^{2}(\Omega)\right)$ в силу эллиптической регулярности (см., например, [1]). Продолжая по индукции, получаем $v \in H^{s}\left(0, T ; H^{s}(\Omega)\right)$, что доказьвает лемму 10.

ЗАмечАнИЕ 5. Пусть в условиях леммы 10 выполнены равенства $\operatorname{div}_{y} k_{0}^{0}=0$, $\operatorname{div}_{y} A_{0} a_{0}^{0}=0$ и $\operatorname{div}_{y} A_{0} b_{0}^{0}=0$ (например, соответствующие коэффициенты являются постоянными). Тогда в соответствии с (2.25) и (2.28) соотношения (2.22) выполнены при $c_{2}=0$. В этом случае непосредственно проверяется, что постоянные $C$ в неравенствах (2.32) и (2.33) не зависят от $\vartheta$, и поэтому выполнен аналог леммы 8 для задачи (1.24). Эти постоянные также можно считать не зависящими от $\vartheta$ при дополнительном условии $\vartheta \leqslant C<\infty$, но в общем случае оценки $(2.32)$ и (2.33) не являются равномерными по $\vartheta$.

\section{§3. Доказательство теоремы 1}

Как уже отмечалось в $\S 2$, однозначно определены $N_{a}, N_{b} \in H_{P}^{1}\left(Y_{1}\right)$ и $N_{1} \in$ $H_{P}^{1}\left(Y_{1}\right)^{n}$, удовлетворяющие (1.14) и (1.12). Следовательно, определены следы $N_{a}, N_{b}$ и $N_{1}$ на $S$ как элементы $H_{P}^{1 / 2}(S)$ и $H_{P}^{1 / 2}(S)^{n}$. Продолжим $N_{a}, N_{b}$ и $N_{1}$ на $Y_{0}$ как 1-периодические решения задач Дирихле:

$$
\begin{aligned}
& -\operatorname{div}_{y}\left(A_{0} \nabla_{y} N_{a}\right)=\operatorname{div}_{y}\left(A_{0} a_{0}\right) \text { в } Y_{0}, \quad N_{a}=N_{a} \text { на } S, \\
& -\operatorname{div}_{y}\left(A_{0} \nabla_{y} N_{b}\right)=\operatorname{div}_{y}\left(A_{0} b_{0}\right) \text { в } Y_{0}, \quad N_{b}=N_{b} \text { на } S \text {, } \\
& -\operatorname{div}_{y}\left(A_{0} \nabla_{y} N_{1}\right)=\operatorname{div}_{y} A_{0} \quad \text { в } Y_{0}, \quad N_{1}=N_{1} \quad \text { на } S .
\end{aligned}
$$

Тогда $N_{a}, N_{b} \in H_{P}^{1}(Y)$ и $N_{1} \in H_{P}^{1}(Y)^{n}$ (см., например, [1]). Определим $Q, P \in$ $H_{P}^{1}(Y)$ как 1-периодические решения задач Дирихле на $Y_{0}$ :

$$
\begin{array}{rllll}
-\operatorname{div}_{y}\left(A_{0} \nabla_{y} Q\right)=m_{0} \quad \text { в } \quad Y_{0}, & Q=0 & \text { на } \bar{Y}_{1}, \\
-\operatorname{div}_{y}\left(A_{0} \nabla_{y} P\right)=r_{0} \quad \text { в } \quad Y_{0}, & P=0 & \text { на } \bar{Y}_{1} .
\end{array}
$$

В случае когда выполнено условие (1.1), начальные члены асимптотического разложения решения задачи (1.4) задаются равенством

$$
u_{a}=v+\varepsilon\left(N_{1}\right)_{\varepsilon} \nabla v+\varepsilon\left(N_{a}\right)_{\varepsilon} v+\varepsilon\left(N_{b}\right)_{\varepsilon} g-\frac{\varepsilon^{2}}{\sigma} Q_{\varepsilon} v_{t}^{\prime}+\frac{\varepsilon^{2}}{\sigma} P_{\varepsilon} f
$$

где $v$ - решение осредненной задачи (1.16) и, например, $P_{\varepsilon}=P(x / \varepsilon)$ обозначает $\varepsilon$-периодическое продолжение этой функции на $\Omega$ (см. $\S 2)$. Следующая хорошо известная формула и различные ее вариации в обозначениях часто используются в дальнейшем

$$
\nabla\left(P_{\varepsilon} f\right)=\varepsilon^{-1}\left(\nabla_{y} P\right)_{\varepsilon} f+P_{\varepsilon} \nabla f
$$


В (3.3) все слагаемые, кроме второго, удовлетворяют однородным граничным условиям Дирихле на $\partial \Omega$. Полезно подправить и это второе слагаемое. Для этого определим гладкую в $\Omega$ функцию $\eta$, равную нулю при $\operatorname{dist}(x, \partial \Omega) \leqslant \varepsilon$ и единице при $\operatorname{dist}(x, \partial \Omega) \geqslant 2 \varepsilon$, где $\operatorname{dist}(x, \partial \Omega)$ обозначает расстояние от точки $x$ до границы $\partial \Omega$. Для достаточно малых $\varepsilon\left(\leqslant \varepsilon_{0}\right)$ такие функции существуют, $\varepsilon|\nabla \eta| \leqslant C$ в $\Omega$ и носители функций $\eta-1$ и $\nabla \eta$ содержатся в приграничной полосе $\Omega_{2 \varepsilon} \subset \Omega$ ширины $2 \varepsilon$ (см., например, [2]). Из ограниченности области $\Omega$ и известной оценки $\operatorname{mes}\left(\Omega_{2 \varepsilon}\right) \leqslant C \varepsilon$ следуют неравенства

$$
\begin{gathered}
\left\|\left(N_{1}\right)_{\varepsilon}\right\|_{L^{2}(\Omega)^{n}}^{2}+\left\|\left(\nabla_{y} N_{1}\right)_{\varepsilon}\right\|_{L^{2}(\Omega)^{n \times n}}^{2} \leqslant C, \\
\left\|\left(N_{1}\right)_{\varepsilon}\right\|_{L^{2}\left(\Omega_{2 \varepsilon}\right)^{n}}^{2}+\left\|\left(\nabla_{y} N_{1}\right)_{\varepsilon}\right\|_{L^{2}\left(\Omega_{2 \varepsilon}\right)^{n \times n}}^{2} \leqslant C \varepsilon
\end{gathered}
$$

и аналогичные неравенства для $N_{a}, N_{b}, Q$ и $P$ (см., например, [9]).

Подправленная асимптотика задается равенством

$$
v_{a}=v+\varepsilon\left(N_{1}\right)_{\varepsilon} \eta \nabla v+\varepsilon\left(N_{a}\right)_{\varepsilon} v+\varepsilon\left(N_{b}\right)_{\varepsilon} g-\frac{\varepsilon^{2}}{\sigma} Q_{\varepsilon} v_{t}^{\prime}+\frac{\varepsilon^{2}}{\sigma} P_{\varepsilon} f .
$$

Учитывая (3.3) и (3.4), имеем $v_{a}-u_{a}=\varepsilon\left(N_{1}\right)_{\varepsilon}(\eta-1) \nabla v$,

$$
\nabla\left(v_{a}-u_{a}\right)=\left(\nabla_{y} N_{1}\right)_{\varepsilon}(\eta-1) \nabla v+\varepsilon\left(N_{1}\right)_{\varepsilon}(\nabla \eta) \nabla v+\varepsilon\left(N_{1}\right)_{\varepsilon}(\eta-1) \nabla^{2} v .
$$

Следовательно, используя второе неравенство из (3.5) и гладкость $v$, получаем

$$
\left\|v_{a}-u_{a}\right\|_{L^{2}\left(0, T ; L^{2}(\Omega)\right)}^{2}+\left\|\nabla\left(v_{a}-u_{a}\right)\right\|_{L^{2}\left(0, T ; L^{2}(\Omega)^{n}\right)}^{2} \leqslant C \varepsilon,
$$

так что $v_{a}$ достаточно близка к асимптотике $u_{a}$ и $v_{a}=0$ на $\partial \Omega \times(0, T)$.

Введем обозначения

$$
r=u-v_{a}, \quad r_{a}=v_{a}-u_{a},
$$

где $u$ - решение задачи (1.4). Выберем $\varphi=r$ в интегральном тождестве (1.11) и вычтем соответствуюшие интегралы от $v_{a}$. Тогда

$$
\begin{aligned}
& \int_{\Omega} m_{\varepsilon} r_{t}^{\prime} r d x+\int_{\Omega}\left(A_{\varepsilon}^{\sigma}\left(\nabla r+a_{\varepsilon} r\right), \nabla r\right) d x+\int_{\Omega}\left(k_{\varepsilon}^{\sigma} \nabla r\right) r d x \\
& =\int_{\Omega} r_{\varepsilon} f r d x-\int_{\Omega} m_{\varepsilon}\left(v_{a}\right)_{t}^{\prime} r d x-\int_{\Omega}\left(k_{\varepsilon}^{\sigma} \nabla v_{a}\right) r d x \\
& \quad-\int_{\Omega}\left(A_{\varepsilon}^{\sigma}\left(\nabla v_{a}+a_{\varepsilon} v_{a}+b_{\varepsilon} g\right), \nabla r\right) d x \equiv I .
\end{aligned}
$$

Повторяя доказательство леммы 1 и учитывая гладкость $v$, заключаем, что

$$
\begin{aligned}
E(r) \equiv & \max _{0 \leqslant t \leqslant T}\|r\|_{L^{2}(\Omega)}^{2}+\sigma \int_{0}^{T}\|\nabla r\|_{L^{2}\left(\Omega_{0}^{\varepsilon}\right)^{n}}^{2} d t \\
& +\int_{0}^{T}\|\nabla r\|_{L^{2}\left(\Omega_{1}^{\varepsilon}\right)^{n}}^{2} d t \leqslant C\left\|\left.r\right|_{t=0}\right\|_{L^{2}(\Omega)}^{2}+C \int_{0}^{T}|I| d t .
\end{aligned}
$$

В силу представления (3.6) и первого неравенства из (3.5) имеем

$$
\max _{0 \leqslant t \leqslant T}\|u-v\|_{L^{2}(\Omega)}^{2} \leqslant \max _{0 \leqslant t \leqslant T}\|r\|_{L^{2}(\Omega)}^{2}+C\left(\varepsilon+\frac{\varepsilon^{2}}{\sigma}\right)
$$


и $\left\|\left.r\right|_{t=0}\right\|_{L^{2}(\Omega)}^{2} \leqslant C\left(\varepsilon+\varepsilon^{2} / \sigma\right)$. Таким образом, для доказательства теоремы 1 остается оценить второе слагаемое в правой части неравенства (3.9) величиной $C\left(\varepsilon+\sigma+\varepsilon^{2} / \sigma\right)$.

Используя (3.4) и (3.8), получаем

$$
\begin{aligned}
\nabla v_{a}= & \nabla r_{a}+\nabla u_{a}=\nabla r_{a}+\nabla v+\left(\nabla_{y} N_{1}\right)_{\varepsilon} \nabla v+\left(\nabla_{y} N_{a}\right)_{\varepsilon} v \\
& +\left(\nabla_{y} N_{b}\right)_{\varepsilon} g-\frac{\varepsilon}{\sigma}\left(\nabla_{y} Q\right)_{\varepsilon} v_{t}^{\prime}+\frac{\varepsilon}{\sigma}\left(\nabla_{y} P\right)_{\varepsilon} f+\varepsilon\left(N_{1}\right)_{\varepsilon} \nabla^{2} v \\
& +\varepsilon\left(N_{a}\right)_{\varepsilon} \nabla v+\varepsilon\left(N_{b}\right)_{\varepsilon} g-\frac{\varepsilon^{2}}{\sigma} Q_{\varepsilon} \nabla v_{t}^{\prime}+\frac{\varepsilon^{2}}{\sigma} P_{\varepsilon} \nabla f \\
\equiv & \nabla r_{a}+\nabla v+\left(\nabla_{y} N_{1}\right)_{\varepsilon} \nabla v+\left(\nabla_{y} N_{a}\right)_{\varepsilon} v \\
& +\left(\nabla_{y} N_{b}\right)_{\varepsilon} g-\frac{\varepsilon}{\sigma}\left(\nabla_{y} Q\right)_{\varepsilon} v_{t}^{\prime}+\frac{\varepsilon}{\sigma}\left(\nabla_{y} P\right)_{\varepsilon} f+\varepsilon J_{1}+\frac{\varepsilon^{2}}{\sigma} J_{2} .
\end{aligned}
$$

Здесь и далее $\left\|J_{s}\right\|_{L^{2}\left(0, T ; L^{2}(\Omega)\right)} \leqslant C$ в силу первого неравенства из $(3.5)$ и гладкости $v, g$ и $f$. По определению $Q_{\varepsilon}$ и $P_{\varepsilon}$ равны нулю на $\Omega_{1}^{\varepsilon}$, следовательно,

$$
A_{\varepsilon}^{\sigma}\left(\nabla v_{a}+a_{\varepsilon} v_{a}+b_{\varepsilon} g\right)=A_{\varepsilon}^{\sigma} \nabla r_{a}+\varepsilon J_{3}+(\widetilde{A})_{\varepsilon} \nabla v+\left(\widetilde{A}_{a}\right)_{\varepsilon} v+\left(\widetilde{A}_{b}\right)_{\varepsilon} g \text { в } \Omega_{1}^{\varepsilon}
$$

где $\widetilde{A}(y)=A_{1}+A_{1} \nabla_{y} N_{1}, \widetilde{A}_{a}(y)=A_{1} a_{1}+A_{1} \nabla_{y} N_{a}$ и $\widetilde{A}_{b}(y)=A_{1} b_{1}+A_{1} \nabla_{y} N_{b}$ удовлетворяют соотношениям

$$
\begin{aligned}
& \langle\widetilde{A}\rangle_{\mathbf{1}}=A, \quad\left\langle\widetilde{A}_{a}\right\rangle_{\mathbf{1}}=A a, \quad\left\langle\widetilde{A}_{b}\right\rangle_{\mathbf{1}}=A b, \\
& \operatorname{div}_{y} \widetilde{A}=0, \quad \operatorname{div}_{y} \widetilde{A}_{a}=0, \quad \operatorname{div}_{y} \widetilde{A}_{b}=0 \quad \text { в } Y_{1} \text {, } \\
& (\widetilde{A}, \nu)=0, \quad\left(\widetilde{A}_{a}, \nu\right)=0, \quad\left(\widetilde{A}_{b}, \nu\right)=0 \text { на } S
\end{aligned}
$$

в соответствии с (1.12)-(1.15). Аналогично получаем

$$
k_{\varepsilon}^{\sigma} \nabla v_{a}=k_{\varepsilon}^{\sigma} \nabla r_{a}+\varepsilon k_{\varepsilon}^{\sigma} J_{1}+(\widetilde{K})_{\varepsilon} \nabla v+\left(\widetilde{k}_{a}\right)_{\varepsilon} v+\left(\widetilde{k}_{b}\right)_{\varepsilon} g \text { в } \Omega_{1}^{\varepsilon},
$$

где $\widetilde{K}(y)=k_{1}+k_{1} \nabla_{y} N_{1}, \widetilde{k}_{a}(y)=k_{1} \nabla_{y} N_{a}$ и $\widetilde{k}_{b}(y)=k_{1} \nabla_{y} N_{b}$ удовлетворяют соотношениям

$$
\langle\widetilde{K}\rangle_{\mathbf{1}}=K, \quad\left\langle\widetilde{k}_{a}\right\rangle_{\mathbf{1}}=k_{a}, \quad\left\langle\widetilde{k}_{b}\right\rangle_{\mathbf{1}}=k_{b}
$$

Соответствующие интегралы по $\Omega$, содержащие $\nabla r_{a}, J_{1}$ и $J_{3}$, не существенны в (3.9), поскольку, например,

$$
\begin{aligned}
\int_{0}^{T} & \left|\int_{\Omega}\left(A_{\varepsilon}^{\sigma} \nabla r_{a}, \nabla r\right) d x\right| d t \\
\leqslant & \frac{\sigma \delta C}{2} \int_{0}^{T}\|\nabla r\|_{L^{2}\left(\Omega_{0}^{\varepsilon}\right)^{n}}^{2} d t+\frac{\delta C}{2} \int_{0}^{T}\|\nabla r\|_{L^{2}\left(\Omega_{1}^{\varepsilon}\right)^{n}}^{2} d t \\
& \quad+\frac{\sigma C}{2 \delta} \int_{0}^{T}\left\|\nabla r_{a}\right\|_{L^{2}\left(\Omega_{0}^{\varepsilon}\right)^{n}}^{2} d t+\frac{C}{2 \delta} \int_{0}^{T}\left\|\nabla r_{a}\right\|_{L^{2}\left(\Omega_{1}^{\varepsilon}\right)^{n}}^{2} d t
\end{aligned}
$$


(см. доказательство неравенства (2.2)). Выбрав $\delta=1 / C$, интегралы с $\nabla r$ справа в (3.15) перенесем в левую часть (3.9), а интегралы с $\nabla r_{a}$ оценим величиной $C \varepsilon$ в силу (3.7). Так же просто оценивается в (3.9) интеграл по $\Omega_{0}^{\varepsilon}$, содержаший $k_{\varepsilon}^{\sigma}$ :

$$
\begin{aligned}
\int_{0}^{T}\left|\int_{\Omega_{0}^{\varepsilon}}\left(k_{\varepsilon}^{\sigma} \nabla v_{a}\right) r d x\right| d t & \leqslant \sigma C \int_{0}^{T}\left\|\nabla v_{a}\right\|_{L^{2}(\Omega)^{n}}\|r\|_{L^{2}(\Omega)} d t \\
& \leqslant \sigma C \int_{0}^{T}\left\|\nabla v_{a}\right\|_{L^{2}(\Omega)^{n}} d t \leqslant C(\sigma+\varepsilon)
\end{aligned}
$$

в силу леммы 1 , представления (3.10) для $\nabla v_{a}$ и определений (1.6). Аналогично, $\left(v_{a}\right)_{t}^{\prime}=v_{t}^{\prime}+\varepsilon J_{4}+\left(\varepsilon^{2} / \sigma\right) J_{5}$, где интегралы по $\Omega$, содержашие $J_{4}$ и $J_{5}$, не существенны в (3.9).

Выделяя сингулярное слагаемое в (3.10), введем обозначение

$$
v_{2}=\frac{\varepsilon}{\sigma}\left(\nabla_{y} P\right)_{\varepsilon} f-\frac{\varepsilon}{\sigma}\left(\nabla_{y} Q\right)_{\varepsilon} v_{t}^{\prime}
$$

и определим $v_{3}$ из равенства

$$
A_{\varepsilon}^{\sigma}\left(\nabla v_{a}+a_{\varepsilon} v_{a}+b_{\varepsilon} g\right)=A_{\varepsilon}^{\sigma} v_{2}+A_{\varepsilon}^{\sigma} v_{3} \text { в } \Omega_{0}^{\varepsilon} .
$$

Непосредственно из (1.6), (3.5), (3.7) и (3.10) заключаем, что

$$
\int_{0}^{T}\left|\int_{\Omega_{0}^{\varepsilon}}\left(A_{\varepsilon}^{\sigma} v_{3}, \nabla r\right) d x\right| d t \leqslant \frac{\sigma \delta C}{2} \int_{0}^{T}\|\nabla r\|_{L^{2}\left(\Omega_{0}^{\varepsilon}\right)^{n}}^{2} d t+\frac{\sigma C}{2 \delta}
$$

(см. $(2.2))$, и, выбрав $\delta=1 / C$, интеграл с $\nabla r$ справа в (3.18) перенесем в левую часть (3.9).

Таким образом, учитывая (3.11), (3.13), (3.15), (3.16) и (3.18), неравенство (3.9) можно переписать в виде

$$
\begin{aligned}
E(r) \leqslant C & \int_{0}^{T} \mid \int_{\Omega_{0}^{\varepsilon}}\left(r_{0}\right)_{\varepsilon} f r d x-\int_{\Omega_{0}^{\varepsilon}}\left(m_{0}\right)_{\varepsilon} v_{t}^{\prime} r d x \\
& -\int_{\Omega_{0}^{\varepsilon}}\left(A_{\varepsilon}^{\sigma} v_{2}, \nabla r\right) d x+\int_{\Omega_{1}^{\varepsilon}}\left(r_{1}\right)_{\varepsilon} f r d x-\int_{\Omega_{1}^{\varepsilon}}\left(m_{1}\right)_{\varepsilon} v_{t}^{\prime} r d x \\
& -\int_{\Omega_{1}^{\varepsilon}}\left((\widetilde{A})_{\varepsilon} \nabla v+\left(\widetilde{A}_{a}\right)_{\varepsilon} v+\left(\widetilde{A}_{b}\right)_{\varepsilon} g, \nabla r\right) d x \\
& -\int_{\Omega_{1}^{\varepsilon}}\left((\widetilde{K})_{\varepsilon} \nabla v+\left(\widetilde{k}_{a}\right)_{\varepsilon} v+\left(\widetilde{k}_{b}\right)_{\varepsilon} g\right) r d x \mid d t+C\left(\varepsilon+\sigma+\frac{\varepsilon^{2}}{\sigma}\right) .
\end{aligned}
$$

Учитьвая граничные условия из (1.4) и определение функции $r$, на границе $\partial \Omega$ имеем равенство $r=0$. Кроме того, гранища $S_{\varepsilon} \equiv \partial \Omega_{1}^{\varepsilon} \backslash \partial \Omega$ имеет класс $C^{2}$, и поэтому в области $\Omega_{1}^{\varepsilon}$ выполнена следуюшая формула интегрирования по частям:

$$
\int_{\Omega_{1}^{\varepsilon}}\left(\left(\widetilde{A}_{a}\right)_{\varepsilon} v, \nabla r\right) d x=\int_{S_{\varepsilon}}\left(\widetilde{A}_{a}, \nu\right)_{\varepsilon} v r d s-\int_{\Omega_{1}^{\varepsilon}} \operatorname{div}\left(\left(\widetilde{A}_{a}\right)_{\varepsilon} v\right) r d x
$$


(см. формулу (2.4) и [14]). Отметим, что $\left(\widetilde{A}_{a}, \nu\right)_{\varepsilon}=0$ на $S_{\varepsilon}$ в соответствии с (3.12). Для оператора дивергенции формула (3.4) имеет вид

$$
\operatorname{div}\left(\left(\widetilde{A}_{a}\right)_{\varepsilon} v\right)=\varepsilon^{-1}\left(\operatorname{div}_{y} \widetilde{A}_{a}\right)_{\varepsilon} v+\left(\widetilde{A}_{a}\right)_{\varepsilon} \nabla v
$$

Используя (3.12), (3.20), заключаем, что

$$
\begin{aligned}
& -\int_{\Omega_{1}^{\varepsilon}}\left((\widetilde{A})_{\varepsilon} \nabla v+\left(\widetilde{A}_{a}\right)_{\varepsilon} v+\left(\widetilde{A}_{b}\right)_{\varepsilon} g, \nabla r\right) d x \\
& \quad=\int_{\Omega_{1}^{\varepsilon}}\left((\widetilde{A})_{\varepsilon} \nabla^{2} v+\left(\widetilde{A}_{a}\right)_{\varepsilon} \nabla v+\left(\widetilde{A}_{b}\right)_{\varepsilon} \nabla g\right) r d x .
\end{aligned}
$$

Для области $\Omega_{0}^{\varepsilon}$ также выполнена формула интегрирования по частям (3.20), изменяется только знак у нормали $\nu_{\varepsilon}$ в соответствии с равенством $\partial \Omega_{0}^{\varepsilon} \backslash \partial \Omega=$ $\partial \Omega_{1}^{\varepsilon} \backslash \partial \Omega=S_{\varepsilon}$. Следовательно, учитьвая (1.6), (3.2), (3.17) и (3.21), получаем

$$
\begin{aligned}
-\int_{\Omega_{0}^{\varepsilon}}\left(A_{\varepsilon}^{\sigma} v_{2}, \nabla r\right) d x= & \int_{S_{\varepsilon}}\left(A_{\varepsilon}^{\sigma} v_{2}, \nu_{\varepsilon}\right) r d s+\varepsilon \int_{\Omega_{0}^{\varepsilon}} \operatorname{div}\left(\left(A_{0} \nabla_{y} P\right)_{\varepsilon} f\right) r d x \\
& -\varepsilon \int_{\Omega_{0}^{\varepsilon}} \operatorname{div}\left(\left(A_{0} \nabla_{y} Q\right)_{\varepsilon} v_{t}^{\prime}\right) r d x=\int_{S_{\varepsilon}}\left(A_{\varepsilon}^{\sigma} v_{2}, \nu_{\varepsilon}\right) r d s \\
& +\int_{\Omega_{0}^{\varepsilon}}\left(\operatorname{div}_{y} A_{0} \nabla_{y} P\right)_{\varepsilon} f r d x-\int_{\Omega_{0}^{\varepsilon}}\left(\operatorname{div}_{y} A_{0} \nabla_{y} Q\right)_{\varepsilon} v_{t}^{\prime} r d x \\
& +\varepsilon \int_{\Omega_{0}^{\varepsilon}}\left(\left(A_{0} \nabla_{y} P\right)_{\varepsilon} r, \nabla f\right) d x-\varepsilon \int_{\Omega_{0}^{\varepsilon}}\left(\left(A_{0} \nabla_{y} Q\right)_{\varepsilon} r, \nabla v_{t}^{\prime}\right) d x \\
= & \varepsilon \int_{S_{\varepsilon}}\left(A_{0} \nabla_{y} P, \nu\right)_{\varepsilon} f r d s-\varepsilon \int_{S_{\varepsilon}}\left(A_{0} \nabla_{y} Q, \nu\right)_{\varepsilon} v_{t}^{\prime} r d s \\
& -\int_{\Omega_{0}^{\varepsilon}}\left(r_{0}\right)_{\varepsilon} f r d x+\int_{\Omega_{0}^{\varepsilon}}\left(m_{0}\right)_{\varepsilon} v_{t}^{\prime} r d x+\varepsilon \int_{\Omega_{0}^{\varepsilon}} J_{6} r d x
\end{aligned}
$$

где последний интеграл не существен в (3.19) в силу гладкости $f, v_{t}^{\prime}$ и первого неравенства из (3.5) для $Q$ и $P$. Таким образом, интегралы по $\Omega_{0}^{\varepsilon}$ заменяются в (3.19) на интегралы по $S_{\varepsilon}$ в соответствии с (3.23).

Используя (1.5), перепишем осредненное уравнение из (1.16) в виде

$$
\begin{aligned}
& \left(\frac{\left\langle m_{0}\right\rangle_{\mathbf{0}}}{\theta_{1}}+\frac{\left\langle m_{1}\right\rangle_{\mathbf{1}}}{\theta_{1}}\right) v_{t}^{\prime}-\frac{A}{\theta_{1}}\left(\nabla^{2} v+a \nabla v+b \nabla g\right) \\
& \quad+\frac{K}{\theta_{1}} \nabla v+\frac{k_{a}}{\theta_{1}} v+\frac{k_{b}}{\theta_{1}} g-\left(\frac{\left\langle r_{0}\right\rangle_{\mathbf{0}}}{\theta_{1}}+\frac{\left\langle r_{1}\right\rangle_{\mathbf{1}}}{\theta_{1}}\right) f=0
\end{aligned}
$$

и введем обозначения для функций, имеющих нулевое среднее на $Y_{1}$ :

$$
\begin{array}{clrl} 
& \widehat{r}_{1}(y)=r_{1}(y)-\frac{\left\langle r_{1}\right\rangle_{1}}{\theta_{1}}, & \widehat{m}_{1}(y)=m_{1}(y)-\frac{\left\langle m_{1}\right\rangle_{1}}{\theta_{1}}, \\
\widehat{A}(y)=\widetilde{A}-\frac{A}{\theta_{1}}, & \widehat{A}_{a}(y)=\widetilde{A}_{a}-\frac{A_{a}}{\theta_{1}}, & \widehat{A}_{b}(y)=\widetilde{A}_{b}-\frac{A_{b}}{\theta_{1}}, \\
\widehat{K}(y)=\widetilde{K}-\frac{K}{\theta_{1}}, & \widehat{k}_{a}(y)=\widetilde{k}_{a}-\frac{k_{a}}{\theta_{1}}, & \widehat{k}_{b}(y)=\widetilde{k}_{b}-\frac{k_{b}}{\theta_{1}} .
\end{array}
$$


Умножим (3.24) на $r$, проинтегрируем полученное соотношение по $\Omega_{1}^{\varepsilon}$ и учтем результат в (3.19). Тогда, используя (3.19), (3.22) и (3.23), неравенство (3.9) можно переписать в виде

$$
\begin{aligned}
E(r) \leqslant & C \int_{0}^{T} \mid \varepsilon \int_{S_{\varepsilon}}\left(A_{0} \nabla_{y} P, \nu\right)_{\varepsilon} f r d s-\varepsilon \int_{S_{\varepsilon}}\left(A_{0} \nabla_{y} Q, \nu\right)_{\varepsilon} v_{t}^{\prime} r d s \\
& +\int_{\Omega_{1}^{\varepsilon}}\left(\frac{\left\langle m_{0}\right\rangle_{\mathbf{0}}}{\theta_{1}}\right)_{\varepsilon} v_{t}^{\prime} r d x-\int_{\Omega_{1}^{\varepsilon}}\left(\frac{\left\langle r_{0}\right\rangle_{\mathbf{0}}}{\theta_{1}}\right)_{\varepsilon} f r d x+\int_{\Omega_{1}^{\varepsilon}}\left(\widehat{r}_{1}\right)_{\varepsilon} f r d x \\
& -\int_{\Omega_{1}^{\varepsilon}}\left(\widehat{m}_{1}\right)_{\varepsilon} v_{t}^{\prime} r d x+\int_{\Omega_{1}^{\varepsilon}}\left((\widehat{A})_{\varepsilon} \nabla^{2} v+\left(\widehat{A}_{a}\right)_{\varepsilon} \nabla v+\left(\widehat{A}_{b}\right)_{\varepsilon} \nabla g\right) r d x \\
& -\int_{\Omega_{1}^{\varepsilon}}\left((\widehat{K})_{\varepsilon} \nabla v+\left(\widehat{k}_{a}\right)_{\varepsilon} v+\left(\widehat{k}_{b}\right)_{\varepsilon} g\right) r d x \mid d t+C\left(\varepsilon+\sigma+\frac{\varepsilon^{2}}{\sigma}\right) .
\end{aligned}
$$

Интегралы в (3.26), содержащие функции из (3.25), оцениваются одинаково. Поэтому рассмотрим, например, первый из этих интегралов. Определим $U \in$ $H_{P}^{1}\left(Y_{1}\right) \cap L^{2}\left(Y_{1}\right) / \mathbb{R}$ как 1-периодическое решение задачи Неймана на $Y_{1}$ :

$$
-\operatorname{div}_{y}\left(A_{1} \nabla_{y} U\right)=\widehat{r}_{1} \quad \text { в } Y_{1}, \quad-\left(A_{1} \nabla_{y} U, \nu\right)=0 \text { на } S .
$$

В силу леммы 2 такое решение сушествует и удовлетворяет неравенству

$$
\left\|\left(A_{1} \nabla_{y} U\right)_{\varepsilon}\right\|_{L^{2}\left(\Omega_{1}^{\varepsilon}\right)^{n}} \leqslant C,
$$

поскольку область $\Omega_{1}^{\varepsilon} \subset \Omega$ ограничена. Следовательно, используя (3.20), (3.21) и $(3.27)$, имеем

$$
\begin{aligned}
& \int_{\Omega_{1}^{\varepsilon}}\left(\widehat{r}_{1}\right)_{\varepsilon} f r d x=-\int_{\Omega_{1}^{\varepsilon}}\left(\operatorname{div}_{y} A_{1} \nabla_{y} U\right)_{\varepsilon} f r d x \\
& \quad=\varepsilon \int_{\Omega_{1}^{\varepsilon}}\left(A_{1} \nabla_{y} U\right)_{\varepsilon}(\nabla f) r d x-\varepsilon \int_{\Omega_{1}^{\varepsilon}} \operatorname{div}\left(\left(A_{1} \nabla_{y} U\right)_{\varepsilon} f\right) r d x \\
& \quad=\varepsilon \int_{\Omega_{1}^{\varepsilon}}\left(A_{1} \nabla_{y} U\right)_{\varepsilon}(\nabla f) r d x+\varepsilon \int_{\Omega_{1}^{\varepsilon}}\left(\left(A_{1} \nabla_{y} U\right)_{\varepsilon} f, \nabla r\right) d x,
\end{aligned}
$$

так что эти интегралы не существенны в $(3.26)$ в силу леммы 1 , гладкости $f$ и неравенства (3.28). Здесь учтено, что $\left(A_{1} \nabla_{y} U, \nu\right)_{\varepsilon}=0$ на $S_{\varepsilon}$ в силу (3.27).

Обозначим $W=A_{0} \nabla_{y} Q$. Тогда $W \in L^{2}\left(Y_{0}\right)^{n}$ и $\operatorname{div}_{y} W \in L^{2}\left(Y_{0}\right)$ в силу (3.2) и первого неравенства из (3.5) для $Q$. Следовательно, определен след $(W, \nu)$ на $S$ как элемент пространства $H_{P}^{-1 / 2}(S)$ в соответствии с неравенством $(2.5)$ для $Y_{0}$. Интегрируя (3.2) по $Y_{0}$ и затем по частям, получаем

$$
\left\langle m_{0}\right\rangle_{\mathbf{0}}=-\left\langle\operatorname{div}_{y} A_{0} \nabla_{y} Q\right\rangle_{\mathbf{0}}=\int_{S}\left(A_{0} \nabla_{y} Q, \nu\right) d s,
$$

где учтено, что $\nu$ является внутренней нормалью для $Y_{0}$. Далее, $\left\langle\left\langle m_{0}\right\rangle_{\mathbf{0}} / \theta_{1}\right\rangle_{\mathbf{1}}=$ $\left\langle m_{0}\right\rangle_{\mathbf{0}}$ и в силу леммы 2 существует 1-периодическое решение $V \in H_{P}^{1}\left(Y_{1}\right) \cap$ $L^{2}\left(Y_{1}\right) / \mathbb{R}$ задачи Неймана на $Y_{1}$ :

$$
-\operatorname{div}_{y}\left(A_{1} \nabla_{y} V\right)=\frac{\left\langle m_{0}\right\rangle_{\mathbf{0}}}{\theta_{1}} \quad \text { в } Y_{1}, \quad-\left(A_{1} \nabla_{y} V, \nu\right)=\left(A_{0} \nabla_{y} Q, \nu\right) \text { на } S,
$$


удовлетворяюшее неравенству (3.28). Следовательно, используя (3.20), (3.21) и (3.31), имеем

$$
\begin{aligned}
\int_{\Omega_{1}^{\varepsilon}}\left(\frac{\left\langle m_{0}\right\rangle_{\mathbf{0}}}{\theta_{1}}\right)_{\varepsilon} v_{t}^{\prime} r d x=-\int_{\Omega_{1}^{\varepsilon}}\left(\operatorname{div}_{y} A_{1} \nabla_{y} V\right)_{\varepsilon} v_{t}^{\prime} r d x \\
=\varepsilon \int_{\Omega_{1}^{\varepsilon}}\left(A_{1} \nabla_{y} V\right)_{\varepsilon}\left(\nabla v_{t}^{\prime}\right) r d x-\varepsilon \int_{\Omega_{1}^{\varepsilon}} \operatorname{div}\left(\left(A_{1} \nabla_{y} V\right)_{\varepsilon} v_{t}^{\prime}\right) r d x \\
=\varepsilon \int_{\Omega_{1}^{\varepsilon}}\left(A_{1} \nabla_{y} V\right)_{\varepsilon}\left(\nabla v_{t}^{\prime}\right) r d x+\varepsilon \int_{\Omega_{1}^{\varepsilon}}\left(\left(A_{1} \nabla_{y} V\right)_{\varepsilon} v_{t}^{\prime}, \nabla r\right) d x \\
\quad-\varepsilon \int_{S_{\varepsilon}}\left(A_{1} \nabla_{y} V, \nu\right)_{\varepsilon} v_{t}^{\prime} r d s=\varepsilon \int_{S_{\varepsilon}}\left(A_{0} \nabla_{y} Q, \nu\right)_{\varepsilon} v_{t}^{\prime} r d s \\
\quad+\varepsilon \int_{\Omega_{1}^{\varepsilon}}\left(A_{1} \nabla_{y} V\right)_{\varepsilon}\left(\nabla v_{t}^{\prime}\right) r d x+\varepsilon \int_{\Omega_{1}^{\varepsilon}}\left(\left(A_{1} \nabla_{y} V\right)_{\varepsilon} v_{t}^{\prime}, \nabla r\right) d x,
\end{aligned}
$$

где последние два интеграла не существенны в (3.26) (в силу леммы 1 , гладкости $v$ и неравенства (3.28) для $V$ ), а граничный интеграл сокрашается с соответствующим граничным интегралом из (3.26). Оценивая аналогичным образом оставшиеся в (3.26) интегралы, заключаем, что

$$
E(r) \leqslant C\left(\varepsilon+\sigma+\frac{\varepsilon^{2}}{\sigma}\right)
$$

Теорема 1 доказана.

ЗАМЕчАнИЕ 6 . Несложно проверить, что в доказательстве теоремы 1 использовались только конечная гладкость $(2.9)$ с $\varkappa \leqslant 2$ решения осредненной задачи (1.16) и конечная гладкость $f$ и $g$.

Учитывая явньй вид задач (3.1), можно улучшить оценку (3.18). Определяя дополнительные задачи на ячейке, можно улучшить оценку (3.16). Такой подход позволяет увеличить показатели степеней $\sigma$ и $\varepsilon^{2} / \sigma$ в (3.33). Однако без дополнительных предположений не улучшается показатель степени $\varepsilon$ в (3.33), чему препятствует неулучшаемость оценки (3.7), связанная с краевыми эффектами в задаче (1.4) вблизи $\partial \Omega$.

\section{§4. Доказательство теоремы 2}

Как и в доказательстве теоремы 1 , пусть $N_{a}, N_{b} \in H_{P}^{1}(Y)$ и $N_{1} \in H_{P}^{1}(Y)^{n}$ удовлетворяют (1.14), (1.12), (3.1), поэтому и неравенствам (3.5). Пусть функции $Q(t, y)$ и $P(t, y)$ являются 1-периодическими решениями задач (1.17). Тогда из леммы 3 и ограниченности области $\Omega$ следуют неравенства

$$
\begin{gathered}
\left\|Q_{\varepsilon}\right\|_{L^{\infty}\left(0, T ; L^{2}(\Omega)\right)}^{2}+\vartheta\left\|\left(\nabla_{y} Q\right)_{\varepsilon}\right\|_{L^{2}\left(0, T ; L^{2}(\Omega)^{n}\right)}^{2} \leqslant C, \\
\left\|P_{\varepsilon}\right\|_{L^{\infty}\left(0, T ; L^{2}(\Omega)\right)}^{2}+\vartheta\left\|\left(\nabla_{y} P\right)_{\varepsilon}\right\|_{L^{2}\left(0, T ; L^{2}(\Omega)^{n}\right)}^{2} \leqslant C .
\end{gathered}
$$

В этих неравенствах и далее постоянная $C$ не зависит от $\varepsilon, \sigma$ и $\vartheta$.

В случае когда выполнено условие (1.2), начальные члены асимптотического разложения решения задачи (1.4) задаются равенством

$$
u_{a}=v+\varepsilon\left(N_{1}\right)_{\varepsilon} \nabla v+\varepsilon\left(N_{a}\right)_{\varepsilon} v+\varepsilon\left(N_{b}\right)_{\varepsilon} g-Q_{\varepsilon} *\left(v_{t}^{\prime}\right)+P_{\varepsilon} * f,
$$


где $v$ - решение осредненной задачи (1.19). Отметим, что последние два слагаемых в (4.2) определены как элементы пространства $C^{0}\left([0, T] ; H_{0}^{1}(\Omega)\right)$ в силу леммы 5 , неравенств (4.1) и гладкости $v$ и $f$. Определим также подправленную асимптотику $v_{a}$ равенством

$$
v_{a}=u_{a}+\varepsilon\left(N_{1}\right)_{\varepsilon}(\eta-1) \nabla v
$$

(см. §3). Тогда выполнено неравенство (3.7) и $v_{a}=0$ на $\partial \Omega \times(0, T)$.

Введем обозначения (3.8). Тогда выполнены неравенство (3.9), неравенство

$$
\max _{0 \leqslant t \leqslant T}\left\|u-v-P_{\varepsilon} * f+Q_{\varepsilon} *\left(v_{t}^{\prime}\right)\right\|_{L^{2}(\Omega)}^{2} \leqslant \max _{0 \leqslant t \leqslant T}\|r\|_{L^{2}(\Omega)}^{2}+C \varepsilon
$$

и $\left\|\left.r\right|_{t=0}\right\|_{L^{2}(\Omega)}^{2} \leqslant C \varepsilon$ в силу первого неравенства из (3.5). Таким образом, для доказательства теоремы 2 остается оценить второе слагаемое в правой части неравенства (3.9) величиной

$$
C\left(\varepsilon+\varepsilon \sqrt{\vartheta}+\sigma+\frac{\sigma}{\varepsilon \sqrt{\vartheta}}+\frac{\varepsilon}{\sqrt{\sigma \vartheta}}\left|\vartheta-\frac{\sigma}{\varepsilon^{2}}\right|\right) .
$$

При этом интегралы по $\Omega_{1}^{\varepsilon}$ будут оцениваться фактически так же, как и в доказательстве теоремы 1 , а оценки интегралов по $\Omega_{0}^{\varepsilon}$ несколько изменятся.

По определению $Q_{\varepsilon}$ и $P_{\varepsilon}$ равны нулю на $\Omega_{1}^{\varepsilon} \times(0, T)$. Следовательно, выполнены соотношения (3.11)-(3.14), а равенство (3.10) принимает вид

$$
\begin{aligned}
\nabla v_{a}=\nabla & r_{a}+\nabla v+\left(\nabla_{y} N_{1}\right)_{\varepsilon} \nabla v+\left(\nabla_{y} N_{a}\right)_{\varepsilon} v+\left(\nabla_{y} N_{b}\right)_{\varepsilon} g \\
& -\frac{1}{\varepsilon}\left(\nabla_{y} Q\right)_{\varepsilon} *\left(v_{t}^{\prime}\right)+\frac{1}{\varepsilon}\left(\nabla_{y} P\right)_{\varepsilon} * f-Q_{\varepsilon} *\left(\nabla v_{t}^{\prime}\right)+P_{\varepsilon} *(\nabla f)+\varepsilon J_{1} .
\end{aligned}
$$

Здесь и далее $\left\|J_{s}\right\|_{L^{2}\left(0, T ; L^{2}(\Omega)\right)} \leqslant C$ в силу первого неравенства из $(3.5)$ и гладкостей $v, g$ и $f$ с оценками норм, не зависящими от $\vartheta$ (см. лемму 8). Соответствующие интегралы по $\Omega$, содержашие $\nabla r_{a}, J_{1}$ и $J_{3}$ (из (3.11)), не существенны в $(3.9)$, поскольку, например, для интеграла с $\nabla r_{a}$ выполнено (3.15).

Выделяя сингулярное слагаемое в (4.4), введем обозначение

$$
v_{2}=\frac{1}{\varepsilon}\left(\nabla_{y} P\right)_{\varepsilon} * f-\frac{1}{\varepsilon}\left(\nabla_{y} Q\right)_{\varepsilon} *\left(v_{t}^{\prime}\right)
$$

и определим $v_{3}$ из равенства

$$
A_{\varepsilon}^{\sigma}\left(\nabla v_{a}+a_{\varepsilon} v_{a}+b_{\varepsilon} g\right)=A_{\varepsilon}^{\sigma} v_{2}+A_{\varepsilon}^{\sigma} v_{3} \text { в } \Omega_{0}^{\varepsilon}
$$

Непосредственно из (1.6), (3.5), (3.7), (4.1), (4.4) и леммы 5 заключаем, что выполнено неравенство (3.18). При выводе этого неравенства использовано, что $v_{3}$ не содержит градиентов от $Q_{\varepsilon}$ и $P_{\varepsilon}$, оценки которых могут зависеть от $\vartheta$. Так же просто оценивается в $(3.9)$ интеграл по $\Omega_{0}^{\varepsilon}$, содержащий $k_{\varepsilon}^{\sigma}$ :

$$
\begin{aligned}
\int_{0}^{T} \mid & \int_{\Omega_{0}^{\varepsilon}}\left(k_{\varepsilon}^{\sigma} \nabla v_{a}\right) r d x \mid d t \leqslant \sigma C \int_{0}^{T}\left\|v_{2}+\nabla v_{a}-v_{2}\right\|_{L^{2}(\Omega)^{n}}\|r\|_{L^{2}(\Omega)} d t \\
\leqslant & \frac{\sigma C}{\varepsilon} \int_{0}^{T}\left\|\left(\nabla_{y} P\right)_{\varepsilon} * f\right\|_{L^{2}(\Omega)^{n}} d t+\frac{\sigma C}{\varepsilon} \int_{0}^{T}\left\|\left(\nabla_{y} Q\right)_{\varepsilon} *\left(v_{t}^{\prime}\right)\right\|_{L^{2}(\Omega)^{n}} d t \\
& +\sigma C \int_{0}^{T}\left\|\nabla v_{a}-v_{2}\right\|_{L^{2}(\Omega)^{n}} d t \leqslant \frac{\sigma C}{\varepsilon}\left\|\left(\nabla_{y} P\right)_{\varepsilon}\right\|_{L^{2}\left(0, T ; L^{2}(\Omega)^{n}\right)} \\
& +\frac{\sigma C}{\varepsilon}\left\|\left(\nabla_{y} Q\right)_{\varepsilon}\right\|_{L^{2}\left(0, T ; L^{2}(\Omega)^{n}\right)}+\sigma C \leqslant C\left(\frac{\sigma}{\varepsilon \sqrt{\vartheta}}+\sigma\right)
\end{aligned}
$$


(см. доказательство леммы 5), где учтены лемма 1 , неравенства (3.5) и (4.1), гладкость $v, g, f$ и тот факт, что $\nabla v_{a}-v_{2}$ не содержит градиентов от $Q_{\varepsilon}$ и $P_{\varepsilon}$.

Непосредственно из (1.6) и (4.5) следует, что

$$
\begin{aligned}
& \int_{\Omega_{0}^{\varepsilon}}\left(A_{\varepsilon}^{\sigma} v_{2}, \nabla r\right) d x=\frac{\sigma}{\varepsilon} \int_{\Omega_{0}^{\varepsilon}}\left(\left(A_{0} \nabla_{y} P\right)_{\varepsilon} * f, \nabla r\right) d x \\
& \quad-\frac{\sigma}{\varepsilon} \int_{\Omega_{0}^{\varepsilon}}\left(\left(A_{0} \nabla_{y} Q\right)_{\varepsilon} *\left(v_{t}^{\prime}\right), \nabla r\right) d x=\varepsilon \vartheta \int_{\Omega_{0}^{\varepsilon}}\left(\left(A_{0} \nabla_{y} P\right)_{\varepsilon} * f, \nabla r\right) d x \\
& \quad-\varepsilon \vartheta \int_{\Omega_{0}^{\varepsilon}}\left(\left(A_{0} \nabla_{y} Q\right)_{\varepsilon} *\left(v_{t}^{\prime}\right), \nabla r\right) d x+\varepsilon\left(\frac{\sigma}{\varepsilon^{2}}-\vartheta\right) \int_{\Omega_{0}^{\varepsilon}}\left(\left(A_{0} \nabla_{y} P\right)_{\varepsilon} * f, \nabla r\right) d x \\
& \quad-\varepsilon\left(\frac{\sigma}{\varepsilon^{2}}-\vartheta\right) \int_{\Omega_{0}^{\varepsilon}}\left(\left(A_{0} \nabla_{y} Q\right)_{\varepsilon} *\left(v_{t}^{\prime}\right), \nabla r\right) d x
\end{aligned}
$$

где последние два интеграла не существенны в (3.9), поскольку, например,

$$
\begin{aligned}
& \int_{0}^{T}\left|\varepsilon\left(\frac{\sigma}{\varepsilon^{2}}-\vartheta\right) \int_{\Omega_{0}^{\varepsilon}}\left(\left(A_{0} \nabla_{y} P\right)_{\varepsilon} * f, \nabla r\right) d x\right| d t \\
& \quad \leqslant \varepsilon\left|\vartheta-\frac{\sigma}{\varepsilon^{2}}\right|\left\|\left(A_{0} \nabla_{y} P\right)_{\varepsilon} * f\right\|_{L^{2}\left(0, T ; L^{2}(\Omega)^{n}\right)}\|\nabla r\|_{L^{2}\left(0, T ; L^{2}\left(\Omega_{0}^{\varepsilon}\right)^{n}\right)} \\
& \quad \leqslant \frac{\varepsilon C}{\sqrt{\sigma}}\left|\vartheta-\frac{\sigma}{\varepsilon^{2}}\right|\left\|\left(\nabla_{y} P\right)_{\varepsilon}\right\|_{L^{2}\left(0, T ; L^{2}(\Omega)^{n}\right)} \leqslant \frac{\varepsilon C}{\sqrt{\sigma \vartheta}}\left|\vartheta-\frac{\sigma}{\varepsilon^{2}}\right| .
\end{aligned}
$$

Здесь учтены леммы 1 и 5 , неравенство (4.1) и гладкость $f$.

Используя первое равенство из (2.7) и начальные условия из (1.17), получаем

$$
\begin{aligned}
\left(v_{a}\right)_{t}^{\prime} & =v_{t}^{\prime}+\left(P_{t}^{\prime}\right)_{\varepsilon} * f+\left(\left.P\right|_{t=0}\right)_{\varepsilon} f-\left(Q_{t}^{\prime}\right)_{\varepsilon} *\left(v_{t}^{\prime}\right)-\left(\left.Q\right|_{t=0}\right)_{\varepsilon} v_{t}^{\prime}+\varepsilon J_{4} \\
& =\left(P_{t}^{\prime}\right)_{\varepsilon} * f+\left(\frac{r_{0}}{m_{0}}\right)_{\varepsilon} f-\left(Q_{t}^{\prime}\right)_{\varepsilon} *\left(v_{t}^{\prime}\right)+\varepsilon J_{4} \text { в } \Omega_{0}^{\varepsilon}
\end{aligned}
$$

и $\left(v_{a}\right)_{t}^{\prime}=v_{t}^{\prime}+\varepsilon J_{4}$ в $\Omega_{1}^{\varepsilon}$, где интегралы, содержащие $J_{4}$, не существенны в (3.9).

Таким образом, учитывая (3.11), (3.13), (3.15), (3.18), (4.7), (4.8) и (4.10), неравенство (3.9) можно переписать в виде

$$
\begin{aligned}
E(r) \leqslant & C \int_{0}^{T} \mid \int_{\Omega_{0}^{\varepsilon}}\left(m_{0} Q_{t}^{\prime}\right)_{\varepsilon} *\left(v_{t}^{\prime}\right) r d x-\int_{\Omega_{0}^{\varepsilon}}\left(m_{0} P_{t}^{\prime}\right)_{\varepsilon} *(f) r d x \\
& +\varepsilon \vartheta \int_{\Omega_{0}^{\varepsilon}}\left(\left(A_{0} \nabla_{y} Q\right)_{\varepsilon} *\left(v_{t}^{\prime}\right), \nabla r\right) d x-\varepsilon \vartheta \int_{\Omega_{0}^{\varepsilon}}\left(\left(A_{0} \nabla_{y} P\right)_{\varepsilon} *(f), \nabla r\right) d x \\
& -\int_{\Omega_{1}^{\varepsilon}}\left((\widetilde{A})_{\varepsilon} \nabla v+\left(\widetilde{A}_{a}\right)_{\varepsilon} v+\left(\widetilde{A}_{b}\right)_{\varepsilon} g, \nabla r\right) d x-\int_{\Omega_{1}^{\varepsilon}}\left(m_{1}\right)_{\varepsilon} v_{t}^{\prime} r d x \\
& -\int_{\Omega_{1}^{\varepsilon}}\left((\widetilde{K})_{\varepsilon} \nabla v+\left(\widetilde{k}_{a}\right)_{\varepsilon} v+\left(\widetilde{k}_{b}\right)_{\varepsilon} g\right) r d x+\int_{\Omega_{1}^{\varepsilon}}\left(r_{1}\right)_{\varepsilon} f r d x \mid d t \\
& +C\left(\varepsilon+\varepsilon \sqrt{\vartheta}+\sigma+\frac{\sigma}{\varepsilon \sqrt{\vartheta}}+\frac{\varepsilon}{\sqrt{\sigma \vartheta}}\left|\vartheta-\frac{\sigma}{\varepsilon^{2}}\right|\right) .
\end{aligned}
$$


Используя равенство (3.21), уравнения из (1.17) и интегрируя по частям, получаем

$$
\begin{aligned}
& -\varepsilon \vartheta \int_{\Omega_{0}^{\varepsilon}}\left(\left(A_{0} \nabla_{y} P\right)_{\varepsilon} *(f), \nabla r\right) d x=\varepsilon \vartheta \int_{S_{\varepsilon}}\left(\left(A_{0} \nabla_{y} P, \nu\right)_{\varepsilon} * f\right) r d s \\
& \quad+\varepsilon \vartheta \int_{\Omega_{0}^{\varepsilon}} \operatorname{div}\left(\left(A_{0} \nabla_{y} P\right)_{\varepsilon} * f\right) r d x=\varepsilon \vartheta \int_{S_{\varepsilon}}\left(\left(A_{0} \nabla_{y} P, \nu\right)_{\varepsilon} * f\right) r d s \\
& \quad+\vartheta \int_{\Omega_{0}^{\varepsilon}}\left(\left(\operatorname{div}_{y} A_{0} \nabla_{y} P\right)_{\varepsilon} * f\right) r d x+\varepsilon \vartheta \int_{\Omega_{0}^{\varepsilon}}\left(\left(A_{0} \nabla_{y} P\right)_{\varepsilon} *(\nabla f)\right) r d x \\
& =\varepsilon \vartheta \int_{S_{\varepsilon}}\left(\left(A_{0} \nabla_{y} P, \nu\right)_{\varepsilon} * f\right) r d s+\int_{\Omega_{0}^{\varepsilon}}\left(\left(m_{0} P_{t}^{\prime}\right)_{\varepsilon} * f\right) r d x+\varepsilon \int_{\Omega_{0}^{\varepsilon}} J_{5} r d x
\end{aligned}
$$

где последний интеграл не существен в (4.11) в силу леммы 5 , гладкости $f$ и неравенства (4.1) для $P$. Аналогичные (4.12) равенства выполнены для интеграла по $\Omega_{0}^{\varepsilon}$ в $(4.11)$, содержащего $\vartheta$ и $Q$. Таким образом, интегралы по $\Omega_{0}^{\varepsilon}$ заменяются в (4.11) на интегралы по $S_{\varepsilon}$ в соответствии с (4.12).

Учитывая (1.5), перепишем осредненное уравнение из (1.19) в виде

$$
\begin{aligned}
& \frac{\left\langle m_{1}\right\rangle_{1}}{\theta_{1}} v_{t}^{\prime}-\left(\frac{\left\langle m_{0} Q_{t}^{\prime}\right\rangle_{0}}{\theta_{1}}\right) *\left(v_{t}^{\prime}\right)-\frac{A}{\theta_{1}}\left(\nabla^{2} v+a \nabla v+b \nabla g\right) \\
& \quad+\frac{K}{\theta_{1}} \nabla v+\frac{k_{a}}{\theta_{1}} v+\frac{k_{b}}{\theta_{1}} g-\frac{\left\langle r_{1}\right\rangle_{1}}{\theta_{1}} f+\left(\frac{\left\langle m_{0} P_{t}^{\prime}\right\rangle_{0}}{\theta_{1}}\right) * f=0
\end{aligned}
$$

и введем обозначения (3.25) для функций, имеющих нулевое среднее на $Y_{1}$. Умножим (4.13) на $r$, проинтегрируем полученное соотношение по $\Omega_{1}^{\varepsilon}$ и учтем результат и равенство (3.22) в (4.11). Тогда, оценивая интегралы в (4.11), содержашие функции из (3.25), как и в $\S 3$ (см. $(3.27)-(3.29))$, неравенство (4.11) можно переписать в виде

$$
\begin{aligned}
E(r) \leqslant & C \int_{0}^{T} \mid \int_{\Omega_{1}^{\varepsilon}}\left(\frac{\left\langle m_{0} P_{t}^{\prime}\right\rangle_{\mathbf{0}}}{\theta_{1}}\right)_{\varepsilon} * f r d x-\int_{\Omega_{1}^{\varepsilon}}\left(\frac{\left\langle m_{0} Q_{t}^{\prime}\right\rangle_{\mathbf{0}}}{\theta_{1}}\right)_{\varepsilon} *\left(v_{t}^{\prime}\right) r d x \\
& +\varepsilon \vartheta \int_{S_{\varepsilon}}\left(A_{0} \nabla_{y} P, \nu\right)_{\varepsilon} * f r d s-\varepsilon \vartheta \int_{S_{\varepsilon}}\left(A_{0} \nabla_{y} Q, \nu\right)_{\varepsilon} *\left(v_{t}^{\prime}\right) r d s \mid d t \\
& +C\left(\varepsilon+\varepsilon \sqrt{\vartheta}+\sigma+\frac{\sigma}{\varepsilon \sqrt{\vartheta}}+\frac{\varepsilon}{\sqrt{\sigma \vartheta}}\left|\vartheta-\frac{\sigma}{\varepsilon^{2}}\right|\right) .
\end{aligned}
$$

Интегралы в (4.14), содержащие функции $P$ и $Q$, оцениваются одинаково. Поэтому рассмотрим, например, интегралы с $P$. Для оценки этих интегралов непосредственно не применима схема из $\S 3$ (см. (3.30)-(3.32)), поскольку производные по $t$ от решений задачи (1.17) могут иметь особенности и соответствующие следы на $S$ могут быть не определены. Однако свертка по $t$ примечательна тем, что производные по $t$ можно "перебросить" на второй, достаточно гладкий, множитель, и возникшая проблема легко решается.

Пусть $R$ обозначает такую первообразную по $t$ от $P$, что $\left.R\right|_{t=0}=0$. Из равенств (2.7) следует, что

$$
\begin{gathered}
\left\langle m_{0} P_{t}^{\prime}\right\rangle_{\mathbf{0}} * f=\left\langle m_{0}\left(\int_{0}^{t} P_{t}^{\prime} d t\right)_{t}^{\prime}\right\rangle_{\mathbf{0}} * f=\left\langle m_{0} P-r_{0}\right\rangle_{\mathbf{0}} *\left(f_{t}^{\prime}\right), \\
\left(A_{0} \nabla_{y} P, \nu\right)_{\varepsilon} * f=\left(A_{0} \nabla_{y} R, \nu\right)_{\varepsilon} *\left(f_{t}^{\prime}\right) .
\end{gathered}
$$


Интегрируя по $t$ уравнение для $P$ из (1.17), имеем

$$
m_{0} P-r_{0}=\vartheta \operatorname{div}\left(A_{0} \nabla_{y} R\right) \text { в } Y_{0} \times(0, T) .
$$

По определению $P=R_{t}^{\prime}$, и поэтому $R$ удовлетворяет неравенству (4.1) (см. лемму 3 и [4]).

Из неравенств (4.1) для $P$ и $R$ следует, что $W \equiv \vartheta A_{0} \nabla_{y} R \in L^{2}\left(Y_{0}\right)^{n}$ и $\operatorname{div}_{y} W \in$ $L^{2}\left(Y_{0}\right)$ для почти всех $t \in(0, T)$. Поэтому для таких $t \in(0, T)$ определен след $(W, \nu)$ на $S$ как элемент пространства $H_{P}^{-1 / 2}(S)$ и

$$
\|(W, \nu)\|_{H_{P}^{-1 / 2}(S)}^{2} \leqslant C\left(\left\|\vartheta A_{0} \nabla_{y} R\right\|_{L^{2}\left(Y_{0}\right)^{n}}^{2}+\left\|m_{0} P-r_{0}\right\|_{L^{2}\left(Y_{0}\right)}^{2}\right)
$$

в соответствии с неравенством (2.5) для $Y_{0}$. Интегрируя последнее неравенство по $(0, T)$ и учитывая $(4.1)$, имеем

$$
\|(W, \nu)\|_{L^{2}\left(0, T ; H_{P}^{-1 / 2}(S)\right)}^{2} \leqslant C(1+\vartheta) .
$$

Интегрируя (4.16) по $Y_{0}$ и затем по частям, получаем

$$
\left\langle r_{0}-m_{0} P\right\rangle_{\mathbf{0}}=-\vartheta\left\langle\operatorname{div}_{y} A_{0} \nabla_{y} R\right\rangle_{\mathbf{0}}=\vartheta \int_{S}\left(A_{0} \nabla_{y} R, \nu\right) d s .
$$

Эти равенства гарантируют для почти всех $t \in(0, T)$ разрешимость задачи Неймана на $Y_{1}$ :

$$
\begin{aligned}
-\operatorname{div}_{y}\left(A_{1} \nabla_{y} V\right) & =\frac{\left\langle r_{0}-m_{0} P\right\rangle_{\mathbf{0}}}{\theta_{1}} \text { в } Y_{1}, \\
-\left(A_{1} \nabla_{y} V, \nu\right) & =\vartheta\left(A_{0} \nabla_{y} R, \nu\right) \text { на } S .
\end{aligned}
$$

В силу леммы 2 для почти всех $t \in(0, T)$ существует 1-периодическое решение $V \in H_{P}^{1}\left(Y_{1}\right) \cap L^{2}\left(Y_{1}\right) / \mathbb{R}$ задачи (4.18) и

$$
\left\|A_{1} \nabla_{y} V\right\|_{L^{2}\left(Y_{1}\right)^{n}}^{2} \leqslant C\left(\left|\left\langle r_{0}-m_{0} P\right\rangle_{\mathbf{0}}\right|^{2}+\left\|\vartheta\left(A_{0} \nabla_{y} R, \nu\right)\right\|_{H_{P}^{-1 / 2}(S)}^{2}\right) .
$$

Интегрируя последнее неравенство по $(0, T)$ и учитывая $(4.1)$ и (4.17), имеем

$$
\left\|\left(A_{1} \nabla_{y} V\right)_{\varepsilon}\right\|_{L^{2}\left(0, T ; L^{2}\left(\Omega_{1}^{\varepsilon}\right)^{n}\right)} \leqslant C \sqrt{1+\vartheta} \leqslant C(1+\sqrt{\vartheta})
$$

поскольку область $\Omega_{1}^{\varepsilon} \subset \Omega$ ограничена.

Таким образом, используя (3.20), (3.21), (4.15) и (4.18), получаем

$$
\begin{aligned}
- & \int_{\Omega_{1}^{\varepsilon}}\left(\frac{\left\langle m_{0} P_{t}^{\prime}\right\rangle_{\mathbf{0}}}{\theta_{1}}\right)_{\varepsilon} * f r d x=-\int_{\Omega_{1}^{\varepsilon}}\left(\operatorname{div}_{y} A_{1} \nabla_{y} V\right)_{\varepsilon} *\left(f_{t}^{\prime}\right) r d x \\
= & \varepsilon \int_{\Omega_{1}^{\varepsilon}}\left(A_{1} \nabla_{y} V\right)_{\varepsilon} *\left(\nabla f_{t}^{\prime}\right) r d x-\varepsilon \int_{\Omega_{1}^{\varepsilon}} \operatorname{div}\left(\left(A_{1} \nabla_{y} V\right)_{\varepsilon} *\left(f_{t}^{\prime}\right)\right) r d x \\
= & \varepsilon \int_{\Omega_{1}^{\varepsilon}}\left(A_{1} \nabla_{y} V\right)_{\varepsilon} *\left(\nabla f_{t}^{\prime}\right) r d x+\varepsilon \int_{\Omega_{1}^{\varepsilon}}\left(\left(A_{1} \nabla_{y} V\right)_{\varepsilon} *\left(f_{t}^{\prime}\right), \nabla r\right) d x \\
& -\varepsilon \int_{S_{\varepsilon}}\left(A_{1} \nabla_{y} V, \nu\right)_{\varepsilon} *\left(f_{t}^{\prime}\right) r d s=\varepsilon \vartheta \int_{S_{\varepsilon}}\left(A_{0} \nabla_{y} R, \nu\right)_{\varepsilon} *\left(f_{t}^{\prime}\right) r d s \\
& +\varepsilon \int_{\Omega_{1}^{\varepsilon}}\left(A_{1} \nabla_{y} V\right)_{\varepsilon} *\left(\nabla f_{t}^{\prime}\right) r d x+\varepsilon \int_{\Omega_{1}^{\varepsilon}}\left(\left(A_{1} \nabla_{y} V\right)_{\varepsilon} *\left(f_{t}^{\prime}\right), \nabla r\right) d x
\end{aligned}
$$


где последние два интеграла не существенны в (4.14) (в силу лемм 1 и 5, гладкости $f$ и неравенства (4.19)), а граничный интеграл сокращается с соответствующим граничным интегралом из (4.14) в силу (4.15). Оценивая аналогичным образом оставшиеся в (4.14) интегралы, заключаем, что

$$
E(r) \leqslant C\left(\varepsilon+\varepsilon \sqrt{\vartheta}+\sigma+\frac{\sigma}{\varepsilon \sqrt{\vartheta}}+\frac{\varepsilon}{\sqrt{\sigma \vartheta}}\left|\vartheta-\frac{\sigma}{\varepsilon^{2}}\right|\right) .
$$

Из условия (1.2) следует, что $\sigma /(\varepsilon \sqrt{\vartheta})=\sqrt{\sigma}(\sqrt{\sigma} / \varepsilon \sqrt{\vartheta})$, где при $\varepsilon \rightarrow 0$ и $\sigma \rightarrow 0$ величина $\sqrt{\sigma} / \varepsilon$ сходится к $\sqrt{\vartheta}$ и поэтому ограничена. По аналогичной причине ограничена и величина $\varepsilon / \sqrt{\sigma}$, что доказывает теорему 2 .

ЗАмЕчАнИЕ 7. Несложно проверить, что в доказательстве теоремы 2 использовалась только конечная гладкость (2.9) решения осредненной задачи (1.19) и конечная гладкость $f$ и $g$.

\section{§5. Доказательство теоремы 4}

Как и в доказательстве теорем 1 и 2 , пусть $N_{a}, N_{b} \in H_{P}^{1}(Y)$ и $N_{1} \in H_{P}^{1}(Y)^{n}$ удовлетворяют $(1.14),(1.12),(3.1)$ и (3.5). Пусть функции $Q(t, y), P(t, y), q(t, y)$ и $p(t, y)$ являются 1-периодическими решениями начально-краевых задач $(1.22)$ и поэтому удовлетворяют неравенствам (4.1). В этих неравенствах и далее постоянные $C$ не зависят от $\varepsilon$ и $\sigma$, но могут зависеть от $\vartheta$.

В случае когда выполнено условие (1.2), начальные члены асимптотического разложения решения задачи (1.4), (1.21) задаются равенством

$$
u_{a}=v+\varepsilon\left(N_{1}\right)_{\varepsilon} \nabla v+\varepsilon\left(N_{a}\right)_{\varepsilon} v+\varepsilon\left(N_{b}\right)_{\varepsilon} g-Q_{\varepsilon} *\left(v_{t}^{\prime}\right)+P_{\varepsilon} * f+q_{\varepsilon} * v+p_{\varepsilon} * g
$$

где $v$ - решение осредненной задачи (1.24), которое сушествует и достаточно гладко в силу леммы 10 . Определим также подправленную асимптотику $v_{a}$ равенством (4.3). Тогда выполнено неравенство (3.7) и $v_{a}=0$ на $\partial \Omega \times(0, T)$.

Введем обозначения (3.8). Тогда выполнены неравенство (3.9), неравенство

$$
\max _{0 \leqslant t \leqslant T}\left\|u-v-P_{\varepsilon} * f+Q_{\varepsilon} *\left(v_{t}^{\prime}\right)-q_{\varepsilon} * v-p_{\varepsilon} * g\right\|_{L^{2}(\Omega)}^{2} \leqslant \max _{0 \leqslant t \leqslant T}\|r\|_{L^{2}(\Omega)}^{2}+C \varepsilon
$$

и $\left\|\left.r\right|_{t=0}\right\|_{L^{2}(\Omega)}^{2} \leqslant C \varepsilon$ в силу первого неравенства из (3.5). Таким образом, для доказательства теоремы 4 остается оценить второе слагаемое в правой части неравенства (3.9) величиной $C\left(\varepsilon+\sqrt{\sigma}+\left|\vartheta-\sigma / \varepsilon^{2}\right|+|\sqrt{\vartheta}-\sqrt{\sigma} / \varepsilon|\right)$. При доказательстве этой оценки будет использоваться схема доказательства из $\S 4$, но с некоторыми изменениями, вызванными наличием дополнительных слагаемых в (1.21) по сравнению с (1.6) и (1.10).

По определению $Q_{\varepsilon}, P_{\varepsilon}, q_{\varepsilon}$ и $p_{\varepsilon}$ равны нулю на $\Omega_{1}^{\varepsilon} \times(0, T)$. Поэтому, как и в $\S 4$, выполнены соотношения (3.11)-(3.14), а равенство (4.4) принимает вид

$$
\begin{aligned}
\nabla v_{a}= & \nabla r_{a}+\nabla v+\left(\nabla_{y} N_{1}\right)_{\varepsilon} \nabla v+\left(\nabla_{y} N_{a}\right)_{\varepsilon} v+\left(\nabla_{y} N_{b}\right)_{\varepsilon} g \\
& -\frac{1}{\varepsilon}\left(\nabla_{y} Q\right)_{\varepsilon} *\left(v_{t}^{\prime}\right)+\frac{1}{\varepsilon}\left(\nabla_{y} P\right)_{\varepsilon} * f-Q_{\varepsilon} *\left(\nabla v_{t}^{\prime}\right)+P_{\varepsilon} *(\nabla f) \\
& +\frac{1}{\varepsilon}\left(\nabla_{y} q\right)_{\varepsilon} * v+\frac{1}{\varepsilon}\left(\nabla_{y} p\right)_{\varepsilon} * g+q_{\varepsilon} *(\nabla v)+p_{\varepsilon} *(\nabla g)+\varepsilon J_{1} .
\end{aligned}
$$


Здесь и далее соответствуюшие интегралы по $\Omega$, содержашие $\nabla r_{a}$ и $J_{s}$, не сушественны в (3.9) (см., например, (3.15)).

Выделяя сингулярные слагаемые в (5.2), введем обозначения (4.5) и

$$
v_{4}=\frac{1}{\varepsilon}\left(\nabla_{y} q\right)_{\varepsilon} * v+\frac{1}{\varepsilon}\left(\nabla_{y} p\right)_{\varepsilon} * g
$$

Обозначим $J_{2}=\left(N_{1}\right)_{\varepsilon} \nabla v+\left(N_{a}\right)_{\varepsilon} v+\left(N_{b}\right)_{\varepsilon} g$ и определим $v_{3}$ из равенства

$$
\begin{aligned}
& A_{\varepsilon}^{\sigma}\left(\nabla v_{a}+a_{\varepsilon} v_{a}+b_{\varepsilon} g\right)=A_{\varepsilon}^{\sigma} v_{2}+A_{\varepsilon}^{\sigma} v_{3}+A_{\varepsilon}^{\sigma} v_{4}+\varepsilon \sqrt{\sigma}\left(A_{0} a_{0}^{0}\right)_{\varepsilon} J_{2} \\
& \quad-\sqrt{\sigma}\left(A_{0} a_{0}^{0} Q\right)_{\varepsilon} *\left(v_{t}^{\prime}\right)+\sqrt{\sigma}\left(A_{0} a_{0}^{0} P\right)_{\varepsilon} * f+\sqrt{\sigma}\left(A_{0} a_{0}^{0} q\right)_{\varepsilon} * v \\
& \quad+\sqrt{\sigma}\left(A_{0} a_{0}^{0} p\right)_{\varepsilon} * g+\sqrt{\sigma}\left(A_{0} a_{0}^{0}\right)_{\varepsilon} v+\sqrt{\sigma}\left(A_{0} b_{0}^{0}\right)_{\varepsilon} g \text { в } \Omega_{0}^{\varepsilon} .
\end{aligned}
$$

Как и в $\S 4$, для слагаемого $A_{\varepsilon}^{\sigma} v_{3}$ в (5.4) вьполнено неравенство (3.18). Аналогично величиной $C \varepsilon$ оценивается в $(3.9)$ интеграл по $\Omega_{0}^{\varepsilon}$, содержаший $\varepsilon \sqrt{\sigma}\left(A_{0} a_{0}^{0}\right)_{\varepsilon} J_{2}$ (см. (2.10)).

Заметим, что для $A_{\varepsilon}^{\sigma} v_{2}$ и $A_{\varepsilon}^{\sigma} v_{4}$ выполнены соотношения (4.8) и (4.9). Эти соотношения фактически означают, что величину $\sigma / \varepsilon$ в (5.4) можно заменить на $\varepsilon \vartheta$ с точностью до не существенных в (3.9) интегралов. Аналогично величину $\sqrt{\sigma}$ в (5.4) можно заменить на $\varepsilon \sqrt{\vartheta}$. Поскольку, например,

$$
\begin{aligned}
& \sqrt{\sigma} \int_{\Omega_{0}^{\varepsilon}}\left(\left(A_{0} a_{0}^{0} Q\right)_{\varepsilon} *\left(v_{t}^{\prime}\right), \nabla r\right) d x=\varepsilon \sqrt{\vartheta} \int_{\Omega_{0}^{\varepsilon}}\left(\left(A_{0} a_{0}^{0} Q\right)_{\varepsilon} *\left(v_{t}^{\prime}\right), \nabla r\right) d x \\
& \quad+\varepsilon\left(\frac{\sqrt{\sigma}}{\varepsilon}-\sqrt{\vartheta}\right) \int_{\Omega_{0}^{\varepsilon}}\left(\left(A_{0} a_{0}^{0} Q\right)_{\varepsilon} *\left(v_{t}^{\prime}\right), \nabla r\right) d x,
\end{aligned}
$$

где последний интеграл не существен в (3.9), имеем

$$
\begin{aligned}
& \int_{0}^{T}\left|\varepsilon\left(\frac{\sqrt{\sigma}}{\varepsilon}-\sqrt{\vartheta}\right) \int_{\Omega_{0}^{\varepsilon}}\left(\left(A_{0} a_{0}^{0} Q\right)_{\varepsilon} *\left(v_{t}^{\prime}\right), \nabla r\right) d x\right| d t \\
& \quad \leqslant \varepsilon\left|\sqrt{\vartheta}-\frac{\sqrt{\sigma}}{\varepsilon}\right|\left\|\left(A_{0} a_{0}^{0} Q\right)_{\varepsilon} *\left(v_{t}^{\prime}\right)\right\|_{L^{2}\left(0, T ; L^{2}(\Omega)^{n}\right)}\|\nabla r\|_{L^{2}\left(0, T ; L^{2}\left(\Omega_{0}^{\varepsilon}\right)^{n}\right)} \\
& \quad \leqslant \frac{\varepsilon C}{\sqrt{\sigma}}\left|\sqrt{\vartheta}-\frac{\sqrt{\sigma}}{\varepsilon}\right|\left\|Q_{\varepsilon}\right\|_{L^{2}\left(0, T ; L^{2}(\Omega)\right)} \leqslant C\left|\sqrt{\vartheta}-\frac{\sqrt{\sigma}}{\varepsilon}\right|
\end{aligned}
$$

в силу лемм 1 и 5 , неравенства (4.1) для $Q_{\varepsilon}$, гладкости $v$ и ограниченности величины $\varepsilon / \sqrt{\sigma}$ при $\varepsilon \rightarrow 0$ и $\sigma \rightarrow 0$.

По определению $k_{\varepsilon}^{\sigma}=\sigma k_{0}+\sqrt{\sigma} k_{0}^{0}$ в $\Omega_{0}^{\varepsilon}$. Для интеграла по $\Omega_{0}^{\varepsilon}$, содержащего $k_{0}$, выполнены неравенства (4.7), и поэтому этот интеграл не сушествен в (3.9). Далее, имеем равенство

$$
\sqrt{\sigma} \int_{\Omega_{0}^{\varepsilon}}\left(k_{0}^{0} \nabla v_{a}\right) r d x=\sqrt{\sigma} \int_{\Omega_{0}^{\varepsilon}} k_{0}^{0}\left(v_{2}+v_{4}\right) r d x+\sqrt{\sigma} \int_{\Omega_{0}^{\varepsilon}} k_{0}^{0}\left(\nabla v_{a}-v_{2}-v_{4}\right) r d x
$$


где последний интеграл не существен в (3.9) в силу неравенств, аналогичных (4.7). Следовательно, можно считать, что

$$
\begin{aligned}
& -\int_{\Omega_{0}^{\varepsilon}}\left(k_{\varepsilon}^{\sigma} \nabla v_{a}\right) r d x=-\sqrt{\sigma} \int_{\Omega_{0}^{\varepsilon}} k_{0}^{0}\left(v_{2}+v_{4}\right) r d x \\
& =\frac{\sqrt{\sigma}}{\varepsilon} \int_{\Omega_{0}^{\varepsilon}}\left(k_{0}^{0} \nabla_{y} Q\right)_{\varepsilon} *\left(v_{t}^{\prime}\right) r d x-\frac{\sqrt{\sigma}}{\varepsilon} \int_{\Omega_{0}^{\varepsilon}}\left(k_{0}^{0} \nabla_{y} P\right)_{\varepsilon} * f r d x \\
& \quad-\frac{\sqrt{\sigma}}{\varepsilon} \int_{\Omega_{0}^{\varepsilon}}\left(k_{0}^{0} \nabla_{y} q\right)_{\varepsilon} * v r d x-\frac{\sqrt{\sigma}}{\varepsilon} \int_{\Omega_{0}^{\varepsilon}}\left(k_{0}^{0} \nabla_{y} p\right)_{\varepsilon} * g r d x .
\end{aligned}
$$

В этих равенствах величину $\sqrt{\sigma} / \varepsilon$ можно заменить величиной $\sqrt{\vartheta}$. Поскольку, например,

$$
\begin{aligned}
& \frac{\sqrt{\sigma}}{\varepsilon} \int_{\Omega_{0}^{\varepsilon}}\left(k_{0}^{0} \nabla_{y} Q\right)_{\varepsilon} *\left(v_{t}^{\prime}\right) r d x=\sqrt{\vartheta} \int_{\Omega_{0}^{\varepsilon}}\left(k_{0}^{0} \nabla_{y} Q\right)_{\varepsilon} *\left(v_{t}^{\prime}\right) r d x \\
& \quad+\left(\frac{\sqrt{\sigma}}{\varepsilon}-\sqrt{\vartheta}\right) \int_{\Omega_{0}^{\varepsilon}}\left(k_{0}^{0} \nabla_{y} Q\right)_{\varepsilon} *\left(v_{t}^{\prime}\right) r d x
\end{aligned}
$$

где последний интеграл не сушествен в (3.9), имеем

$$
\begin{aligned}
& \int_{0}^{T}\left|\left(\frac{\sqrt{\sigma}}{\varepsilon}-\sqrt{\vartheta}\right) \int_{\Omega_{0}^{\varepsilon}}\left(k_{0}^{0} \nabla_{y} Q\right)_{\varepsilon} *\left(v_{t}^{\prime}\right) r d x\right| d t \\
& \quad \leqslant C\left|\sqrt{\vartheta}-\frac{\sqrt{\sigma}}{\varepsilon}\right|\left\|\left(\nabla_{y} Q\right)_{\varepsilon}\right\|_{L^{2}\left(0, T ; L^{2}(\Omega)^{n}\right)} \leqslant C\left|\sqrt{\vartheta}-\frac{\sqrt{\sigma}}{\varepsilon}\right|
\end{aligned}
$$

в силу лемм 1 и 5 , неравенства (4.1) для $Q_{\varepsilon}$ и гладкости $v$.

Используя первое равенство из (2.7) и начальные условия из (1.22), получаем

$$
\begin{aligned}
\left(v_{a}\right)_{t}^{\prime}= & \left(P_{t}^{\prime}\right)_{\varepsilon} * f+\left(\frac{r_{0}}{m_{0}}\right)_{\varepsilon} f-\left(Q_{t}^{\prime}\right)_{\varepsilon} *\left(v_{t}^{\prime}\right)+\left(q_{t}^{\prime}\right)_{\varepsilon} * v+\left(p_{t}^{\prime}\right)_{\varepsilon} * g \\
& +\sqrt{\vartheta}\left(\frac{\operatorname{div}_{y} A_{0} a_{0}^{0}}{m_{0}}\right)_{\varepsilon} v+\sqrt{\vartheta}\left(\frac{\operatorname{div}_{y} A_{0} b_{0}^{0}}{m_{0}}\right)_{\varepsilon} g+\varepsilon J_{3} \text { в } \Omega_{0}^{\varepsilon}
\end{aligned}
$$

и $\left(v_{a}\right)_{t}^{\prime}=v_{t}^{\prime}+\varepsilon J_{3}$ в $\Omega_{1}^{\varepsilon}$, где интегралы по $\Omega$, содержащие $J_{3}$, не существенны в (3.9).

Учитывая (3.15), (3.18), (4.7), (4.8) и (5.4)-(5.9), интегралы по $\Omega_{0}^{\varepsilon}$ в (3.9), содержашие $Q$, можно представить в виде

$$
\begin{gathered}
\int_{\Omega_{0}^{\varepsilon}}\left(m_{0} Q_{t}^{\prime}\right)_{\varepsilon} *\left(v_{t}^{\prime}\right) r d x+\sqrt{\vartheta} \int_{\Omega_{0}^{\varepsilon}}\left(k_{0}^{0} \nabla_{y} Q\right)_{\varepsilon} *\left(v_{t}^{\prime}\right) r d x \\
+\varepsilon \sqrt{\vartheta} \int_{\Omega_{0}^{\varepsilon}}\left(\left(A_{0} \sqrt{\vartheta} \nabla_{y} Q+A_{0} a_{0}^{0} Q\right)_{\varepsilon} *\left(v_{t}^{\prime}\right), \nabla r\right) d x
\end{gathered}
$$


(см. (4.11)). Используя равенство (3.21), уравнения из (1.22) и интегрируя по частям, получаем

$$
\begin{aligned}
\varepsilon \sqrt{\vartheta} \int_{\Omega_{0}^{\varepsilon}}\left(\left(A_{0} \sqrt{\vartheta} \nabla_{y} Q+A_{0} a_{0}^{0} Q\right)_{\varepsilon} *\left(v_{t}^{\prime}\right), \nabla r\right) d x \\
=-\varepsilon \sqrt{\vartheta} \int_{S_{\varepsilon}}\left(\left(A_{0} \sqrt{\vartheta} \nabla_{y} Q+A_{0} a_{0}^{0} Q\right), \nu\right)_{\varepsilon} *\left(v_{t}^{\prime}\right) r d s \\
\quad-\varepsilon \sqrt{\vartheta} \int_{\Omega_{0}^{\varepsilon}} \operatorname{div}\left(\left(A_{0} \sqrt{\vartheta} \nabla_{y} Q+A_{0} a_{0}^{0} Q\right)_{\varepsilon} *\left(v_{t}^{\prime}\right)\right) r d x \\
=-\varepsilon \sqrt{\vartheta} \int_{S_{\varepsilon}}\left(\left(A_{0} \sqrt{\vartheta} \nabla_{y} Q+A_{0} a_{0}^{0} Q\right), \nu\right)_{\varepsilon} *\left(v_{t}^{\prime}\right) r d s \\
\quad-\int_{\Omega_{0}^{\varepsilon}}\left(m_{0} Q_{t}^{\prime}+\sqrt{\vartheta} k_{0}^{0} \nabla_{y} Q\right)_{\varepsilon} *\left(v_{t}^{\prime}\right) r d x+\varepsilon \int_{\Omega_{0}^{\varepsilon}} J_{4} r d x,
\end{aligned}
$$

где последний интеграл не существен в (3.9) в силу леммы 5 , гладкости $v$ и неравенства (4.1) для $Q_{\varepsilon}$. Таким образом, интегралы (5.10) заменяются в (3.9) на интегралы по $S_{\varepsilon}$ в соответствии с (5.11). Точно так же преобразуются интегралы по $\Omega_{0}^{\varepsilon}$ в (3.9), содержашие $P$.

Аналогично, учитывая (3.15), (3.18), (4.7), (4.8) и (5.4)-(5.9), интегралы по $\Omega_{0}^{\varepsilon}$ в (3.9), содержашие $v$, можно представить в виде

$$
\begin{aligned}
& -\int_{\Omega_{0}^{\varepsilon}}\left(m_{0} q_{t}^{\prime}\right)_{\varepsilon} * v r d x-\varepsilon \sqrt{\vartheta} \int_{\Omega_{0}^{\varepsilon}}\left(\left(A_{0} \sqrt{\vartheta} \nabla_{y} q+A_{0} a_{0}^{0} q\right)_{\varepsilon} * v, \nabla r\right) d x \\
& -\sqrt{\vartheta} \int_{\Omega_{0}^{\varepsilon}}\left(k_{0}^{0} \nabla_{y} q\right)_{\varepsilon} * v r d x-\sqrt{\vartheta} \int_{\Omega_{0}^{\varepsilon}}\left(\operatorname{div}_{y} A_{0} a_{0}^{0}\right)_{\varepsilon} v r d x \\
& -\varepsilon \sqrt{\vartheta} \int_{\Omega_{0}^{\varepsilon}}\left(\left(A_{0} a_{0}^{0}\right)_{\varepsilon} v, \nabla r\right) d x
\end{aligned}
$$

и заменить на граничные интегралы

$$
\varepsilon \sqrt{\vartheta} \int_{S_{\varepsilon}}\left(\left(A_{0} \sqrt{\vartheta} \nabla_{y} q+A_{0} a_{0}^{0} q\right), \nu\right)_{\varepsilon} * v r d s+\varepsilon \sqrt{\vartheta} \int_{S_{\varepsilon}}\left(A_{0} a_{0}^{0}, \nu\right)_{\varepsilon} v r d s
$$

Точно так же преобразуются интегралы по $\Omega_{0}^{\varepsilon}$ в (3.9), содержащие $g$.

Используя (2.7) и начальные условия из (1.22), получаем

$$
\begin{aligned}
& \left\langle m_{0} q\right\rangle_{\mathbf{0}} *\left(v_{t}^{\prime}\right)=\left\langle m_{0} q_{t}^{\prime}\right\rangle_{\mathbf{0}} * v+\sqrt{\vartheta}\left\langle\operatorname{div}_{y} A_{0} a_{0}^{0}\right\rangle_{\mathbf{0}} v \\
& \left\langle m_{0} p\right\rangle_{\mathbf{0}} *\left(g_{t}^{\prime}\right)=\left\langle m_{0} p_{t}^{\prime}\right\rangle_{\mathbf{0}} * g+\sqrt{\vartheta}\left\langle\operatorname{div}_{y} A_{0} b_{0}^{0}\right\rangle_{\mathbf{0}} g .
\end{aligned}
$$

Учитывая эти равенства и (1.5), перепишем первое уравнение из (1.24) в виде

$$
\begin{gathered}
\frac{\left\langle m_{1}\right\rangle_{1}}{\theta_{1}} v_{t}^{\prime}-\frac{M_{k}}{\theta_{1}} *\left(v_{t}^{\prime}\right)-\frac{A}{\theta_{1}}\left(\nabla^{2} v+a \nabla v+b \nabla g\right)+\frac{K}{\theta_{1}} \nabla v+\frac{k_{a}}{\theta_{1}} v+\frac{k_{b}}{\theta_{1}} g \\
+\frac{q_{0 k}}{\theta_{1}} * v+\frac{q_{a}}{\theta_{1}} v+\frac{p_{0 k}}{\theta_{1}} * g+\frac{p_{b}}{\theta_{1}} g-\frac{\left\langle r_{1}\right\rangle_{\mathbf{1}}}{\theta_{1}} f+\frac{R_{k}}{\theta_{1}} * f=0,
\end{gathered}
$$


где

$M_{k}=\left\langle m_{0} Q_{t}^{\prime}+\sqrt{\vartheta} k_{0}^{0} \nabla_{y} Q\right\rangle_{\mathbf{0}}, \quad q_{0 k}=\left\langle m_{0} q_{t}^{\prime}+\sqrt{\vartheta} k_{0}^{0} \nabla_{y} q\right\rangle_{\mathbf{0}}, \quad q_{a}=\sqrt{\vartheta}\left\langle\operatorname{div}_{y} A_{0} a_{0}^{0}\right\rangle_{\mathbf{0}}$, $R_{k}=\left\langle m_{0} P_{t}^{\prime}+\sqrt{\vartheta} k_{0}^{0} \nabla_{y} P\right\rangle_{\mathbf{0}}, \quad p_{0 k}=\left\langle m_{0} p_{t}^{\prime}+\sqrt{\vartheta} k_{0}^{0} \nabla_{y} p\right\rangle_{\mathbf{0}}, \quad p_{b}=\sqrt{\vartheta}\left\langle\operatorname{div}_{y} A_{0} b_{0}^{0}\right\rangle_{\mathbf{0}}$.

Введем обозначения (3.25) для функций, имеющих нулевое среднее на $Y_{1}$. Умножим (5.14) на $r$, проинтегрируем полученное соотношение по $\Omega_{1}^{\varepsilon}$ и учтем результат и равенство (3.22) в (3.9). Тогда, оценивая интегралы в (3.9), содержащие функции из $(3.25)$, как и в $\S 3$ (см. (3.27)-(3.29)), неравенство (3.9) можно переписать в виде

$$
\begin{aligned}
E(r) \leqslant & C \int_{0}^{T} \mid \int_{\Omega_{1}^{\varepsilon}}\left(\frac{q_{0 k}}{\theta_{1}}\right)_{\varepsilon} * v r d x+\int_{\Omega_{1}^{\varepsilon}}\left(\frac{q_{a}}{\theta_{1}}\right)_{\varepsilon} v r d x+\int_{\Omega_{1}^{\varepsilon}}\left(\frac{p_{0 k}}{\theta_{1}}\right)_{\varepsilon} * g r d x \\
& -\varepsilon \sqrt{\vartheta} \int_{S_{\varepsilon}}\left(\left(A_{0} \sqrt{\vartheta} \nabla_{y} Q+A_{0} a_{0}^{0} Q\right), \nu\right)_{\varepsilon} *\left(v_{t}^{\prime}\right) r d s-\int_{\Omega_{1}^{\varepsilon}}\left(\frac{M_{k}}{\theta_{1}}\right)_{\varepsilon} *\left(v_{t}^{\prime}\right) r d x \\
& +\varepsilon \sqrt{\vartheta} \int_{S_{\varepsilon}}\left(\left(A_{0} \sqrt{\vartheta} \nabla_{y} P+A_{0} a_{0}^{0} P\right), \nu\right)_{\varepsilon} * f r d s+\int_{\Omega_{1}^{\varepsilon}}\left(\frac{R_{k}}{\theta_{1}}\right)_{\varepsilon} * f r d x \\
& +\varepsilon \sqrt{\vartheta} \int_{S_{\varepsilon}}\left(\left(A_{0} \sqrt{\vartheta} \nabla_{y} q+A_{0} a_{0}^{0} q\right), \nu\right)_{\varepsilon} * v r d s+\varepsilon \sqrt{\vartheta} \int_{S_{\varepsilon}}\left(A_{0} a_{0}^{0}, \nu\right)_{\varepsilon} v r d s \\
& +\varepsilon \sqrt{\vartheta} \int_{S_{\varepsilon}}\left(\left(A_{0} \sqrt{\vartheta} \nabla_{y} p+A_{0} a_{0}^{0} p\right), \nu\right)_{\varepsilon} * g r d s+\varepsilon \sqrt{\vartheta} \int_{S_{\varepsilon}}\left(A_{0} b_{0}^{0}, \nu\right)_{\varepsilon} g r d s \\
& +\int_{\Omega_{1}^{\varepsilon}}\left(\frac{p_{b}}{\theta_{1}}\right)_{\varepsilon} g r d x \mid d t+C\left(\varepsilon+\sqrt{\sigma}+\left|\vartheta-\frac{\sigma}{\varepsilon^{2}}\right|+\left|\sqrt{\vartheta}-\frac{\sqrt{\sigma}}{\varepsilon}\right|\right) .
\end{aligned}
$$

Обозначим $W=\sqrt{\vartheta} A_{0} a_{0}^{0}$. Тогда $W \in L^{2}\left(Y_{0}\right)^{n}$ и $\operatorname{div}_{y} W \in L^{2}\left(Y_{0}\right)$ в силу условий на коэффициенты задачи (1.4), (1.21). Следовательно, определен след $(W, \nu) \in H_{P}^{-1 / 2}(S)$ в соответствии с неравенством (2.5) для $Y_{0}$. Интегрируя по частям, получаем

$$
q_{a}=\sqrt{\vartheta}\left\langle\operatorname{div}_{y} A_{0} a_{0}^{0}\right\rangle_{\mathbf{o}}=-\sqrt{\vartheta} \int_{S}\left(A_{0} a_{0}^{0}, \nu\right) d s .
$$

Тогда в соответствии с леммой 2 существует 1-периодическое решение $U \in$ $H_{P}^{1}\left(Y_{1}\right) \cap L^{2}\left(Y_{1}\right) / \mathbb{R}$ задачи Неймана на $Y_{1}$ :

$$
-\operatorname{div}_{y}\left(A_{1} \nabla_{y} U\right)=\frac{q_{a}}{\theta_{1}} \quad \text { в } Y_{1}, \quad-\left(A_{1} \nabla_{y} U, \nu\right)=-\sqrt{\vartheta}\left(A_{0} a_{0}^{0}, \nu\right) \text { на } S,
$$

удовлетворяющее неравенству (3.28). Следовательно, используя $(3.20),(3.21)$ и (5.16), имеем

$$
\begin{aligned}
\int_{\Omega_{1}^{\varepsilon}}\left(\frac{q_{a}}{\theta_{1}}\right)_{\varepsilon} v r d x=-\int_{\Omega_{1}^{\varepsilon}}\left(\operatorname{div} y A_{1} \nabla_{y} U\right)_{\varepsilon} v r d x \\
=\varepsilon \int_{\Omega_{1}^{\varepsilon}}\left(A_{1} \nabla_{y} U\right)_{\varepsilon}(\nabla v) r d x-\varepsilon \int_{\Omega_{1}^{\varepsilon}} \operatorname{div}\left(\left(A_{1} \nabla_{y} U\right)_{\varepsilon} v\right) r d x \\
=\varepsilon \int_{\Omega_{1}^{\varepsilon}}\left(A_{1} \nabla_{y} U\right)_{\varepsilon}(\nabla v) r d x+\varepsilon \int_{\Omega_{1}^{\varepsilon}}\left(\left(A_{1} \nabla_{y} U\right)_{\varepsilon} v, \nabla r\right) d x \\
-\varepsilon \int_{S_{\varepsilon}}\left(A_{1} \nabla_{y} U, \nu\right)_{\varepsilon} v r d s \\
=-\varepsilon \sqrt{\vartheta} \int_{S_{\varepsilon}}\left(A_{0} a_{0}^{0}, \nu\right)_{\varepsilon} v r d s+\varepsilon \int_{\Omega_{1}^{\varepsilon}} J_{5} r d x
\end{aligned}
$$


где последний интеграл не существен в (5.15) (в силу леммы 1 , гладкости $v$ и неравенства (3.28) для $U$ ), а граничный интеграл сокращается с соответствующим граничным интегралом из (5.15). Аналогично оцениваются последние два интеграла в (5.15).

Пусть $R$ обозначает такую первообразную по $t$ от $P$, что $\left.R\right|_{t=0}=0$. Из равенств (2.7) следует, что

$$
\begin{aligned}
& R_{k} * f=\left\langle m_{0} P_{t}^{\prime}+\sqrt{\vartheta} k_{0}^{0} \nabla_{y} P\right\rangle_{0} * f=\left\langle m_{0} P-r_{0}+\sqrt{\vartheta} k_{0}^{0} \nabla_{y} R\right\rangle_{0} *\left(f_{t}^{\prime}\right) \\
&\left(\left(A_{0} \sqrt{\vartheta} \nabla_{y} P+A_{0} a_{0}^{0} P\right), \nu\right)_{\varepsilon} * f=\left(\left(A_{0} \sqrt{\vartheta} \nabla_{y} R+A_{0} a_{0}^{0} R\right), \nu\right)_{\varepsilon} *\left(f_{t}^{\prime}\right)
\end{aligned}
$$

Интегрируя по $t$ уравнение для $P$ из $(1.22)$, имеем

$$
m_{0} P-r_{0}+\sqrt{\vartheta} k_{0}^{0} \nabla_{y} R=\sqrt{\vartheta} \operatorname{div}\left(A_{0} \sqrt{\vartheta} \nabla_{y} R+A_{0} a_{0}^{0} R\right) \quad \text { в } Y_{0} \times(0, T),
$$

и поэтому $R$ удовлетворяет неравенству (4.1) (см. лемму 3 и [4]). Из этих неравенств следует, что $W \equiv \sqrt{\vartheta}\left(A_{0} \sqrt{\vartheta} \nabla_{y} R+A_{0} a_{0}^{0} R\right) \in L^{2}\left(Y_{0}\right)^{n}$ и $\operatorname{div}_{y} W \in L^{2}\left(Y_{0}\right)$ для почти всех $t \in(0, T)$. Поэтому для таких $t \in(0, T)$ определен след $(W, \nu)$, удовлетворяющий неравенству

$$
\|(W, \nu)\|_{L^{2}\left(0, T ; H_{P}^{-1 / 2}(S)\right)}^{2} \leqslant C .
$$

Интегрируя (5.19) по $Y_{0}$ и затем по частям, получаем

$$
\left\langle r_{0}-m_{0} P-\sqrt{\vartheta} k_{0}^{0} \nabla_{y} R\right\rangle_{0}=\sqrt{\vartheta} \int_{S}\left(\left(A_{0} \sqrt{\vartheta} \nabla_{y} R+A_{0} a_{0}^{0} R\right), \nu\right) d s .
$$

Эти равенства гарантируют для почти всех $t \in(0, T)$ разрешимость задачи Неймана на $Y_{1}$ :

$$
\begin{aligned}
-\operatorname{div}_{y}\left(A_{1} \nabla_{y} V\right) & =\frac{\left\langle r_{0}-m_{0} P-\sqrt{\vartheta} k_{0}^{0} \nabla_{y} R\right\rangle_{0}}{\theta_{1}} \text { в } Y_{1}, \\
-\left(A_{1} \nabla_{y} V, \nu\right) & =\sqrt{\vartheta}\left(\left(A_{0} \sqrt{\vartheta} \nabla_{y} R+A_{0} a_{0}^{0} R\right), \nu\right) \text { на } S .
\end{aligned}
$$

В силу леммы 2 и неравенства (5.20) для почти всех $t \in(0, T)$ сушествует 1-периодическое решение $V \in H_{P}^{1}\left(Y_{1}\right) \cap L^{2}\left(Y_{1}\right) / \mathbb{R}$ задачи (4.18) и

$$
\left\|\left(A_{1} \nabla_{y} V\right)_{\varepsilon}\right\|_{L^{2}\left(0, T ; L^{2}\left(\Omega_{1}^{\varepsilon}\right)^{n}\right)} \leqslant C .
$$

Таким образом, используя (3.20), (3.21), (5.18) и (5.21), получаем

$$
\begin{aligned}
-\int_{\Omega_{1}^{\varepsilon}} & \left(\frac{R_{k}}{\theta_{1}}\right)_{\varepsilon} * f r d x=-\int_{\Omega_{1}^{\varepsilon}}\left(\operatorname{div}_{y} A_{1} \nabla_{y} V\right)_{\varepsilon} *\left(f_{t}^{\prime}\right) r d x \\
= & \varepsilon \int_{\Omega_{1}^{\varepsilon}}\left(A_{1} \nabla_{y} V\right)_{\varepsilon} *\left(\nabla f_{t}^{\prime}\right) r d x-\varepsilon \int_{\Omega_{1}^{\varepsilon}} \operatorname{div}\left(\left(A_{1} \nabla_{y} V\right)_{\varepsilon} *\left(f_{t}^{\prime}\right)\right) r d x \\
= & \varepsilon \int_{\Omega_{1}^{\varepsilon}}\left(A_{1} \nabla_{y} V\right)_{\varepsilon} *\left(\nabla f_{t}^{\prime}\right) r d x+\varepsilon \int_{\Omega_{1}^{\varepsilon}}\left(\left(A_{1} \nabla_{y} V\right)_{\varepsilon} *\left(f_{t}^{\prime}\right), \nabla r\right) d x \\
& -\varepsilon \int_{S_{\varepsilon}}\left(A_{1} \nabla_{y} V, \nu\right)_{\varepsilon} *\left(f_{t}^{\prime}\right) r d s=\varepsilon \int_{\Omega_{1}^{\varepsilon}} J_{6} r d x \\
& +\varepsilon \sqrt{\vartheta} \int_{S_{\varepsilon}}\left(\left(A_{0} \sqrt{\vartheta} \nabla_{y} R+A_{0} a_{0}^{0} R\right)_{, \nu} *\left(f_{t}^{\prime}\right) r d s,\right.
\end{aligned}
$$


где предпоследний интеграл не существен в (5.15) (в силу лемм 1 и 5, гладкости $f$ и неравенства (5.22)), а граничный интеграл сокращается с соответствующим граничным интегралом из (5.15) в силу (5.18). Оценивая аналогичным образом оставшиеся в (5.15) интегралы, заключаем, что

$$
E(r) \leqslant C\left(\varepsilon+\sqrt{\sigma}+\left|\vartheta-\frac{\sigma}{\varepsilon^{2}}\right|+\left|\sqrt{\vartheta}-\frac{\sqrt{\sigma}}{\varepsilon}\right|\right) .
$$

Теорема 4 доказана.

\section{§6. Доказательство теоремы 3}

Из условия (1.3) следует, что $\nu \equiv \sqrt{\sigma} / \varepsilon$ стремится к нулю при $\varepsilon \rightarrow 0$ и $\sigma \rightarrow 0$. Как и в доказательстве теорем 1 и 2 , пусть $N_{a}, N_{b} \in H_{P}^{1}(Y)$ и $N_{1} \in H_{P}^{1}(Y)^{n}$ удовлетворяют $(1.14),(1.12),(3.1)$ и (3.5). Пусть функции $Q(t, y)$ и $P(t, y)$ являются 1-периодическими решениями начально-краевых задач на $Y_{0}$ :

$$
\begin{aligned}
& m_{0} Q_{t}^{\prime}-\nu^{2} \operatorname{div}_{y}\left(A_{0} \nabla_{y} Q\right)=0 \text { в } Y_{0} \times(0, \infty), \\
& Q=0 \text { на } \bar{Y}_{1} \times(0, \infty),\left.\quad Q\right|_{t=0}=1 \text { в } Y_{0}, \\
& m_{0} P_{t}^{\prime}-\nu^{2} \operatorname{div}_{y}\left(A_{0} \nabla_{y} P\right)=0 \text { в } Y_{0} \times(0, \infty), \\
& P=0 \text { на } \bar{Y}_{1} \times(0, \infty),\left.\quad P\right|_{t=0}=\frac{r_{0}}{m_{0}} \text { в } Y_{0},
\end{aligned}
$$

где учтены обозначения (1.2), (1.6) и (1.8). Тогда из леммы 3 и ограниченности области $\Omega$ следуют неравенства

$$
\begin{aligned}
& \left\|Q_{\varepsilon}\right\|_{L^{\infty}\left(0, T ; L^{2}(\Omega)\right)}^{2}+\nu^{2}\left\|\left(\nabla_{y} Q\right)_{\varepsilon}\right\|_{L^{2}\left(0, T ; L^{2}(\Omega)^{n}\right)}^{2} \leqslant C, \\
& \left\|P_{\varepsilon}\right\|_{L^{\infty}\left(0, T ; L^{2}(\Omega)\right)}^{2}+\nu^{2}\left\|\left(\nabla_{y} P\right)_{\varepsilon}\right\|_{L^{2}\left(0, T ; L^{2}(\Omega)^{n}\right)}^{2} \leqslant C .
\end{aligned}
$$

В этих неравенствах и далее постоянная $C$ не зависит от $\varepsilon, \sigma$ и $\nu$.

В случае когда выполнено условие (1.3), начальные члены асимптотического разложения решения задачи (1.4) задаются равенством

$$
u_{a}=v+\varepsilon\left(N_{1}\right)_{\varepsilon} \nabla v+\varepsilon\left(N_{a}\right)_{\varepsilon} v+\varepsilon\left(N_{b}\right)_{\varepsilon} g-Q_{\varepsilon} *\left(v_{t}^{\prime}\right)+P_{\varepsilon} * f
$$

где $v$ - решение осредненной задачи (1.20). Определим также подправленную асимптотику $v_{a}$ равенством (4.3). Тогда выполнено неравенство (3.7) и $v_{a}=0$ на $\partial \Omega \times(0, T)$.

Введем обозначения (3.8). Тогда выполнено неравенство (3.9). После дополнительного исследования свойств решений начально-краевых задач (6.1) правая часть неравенства (3.9) будет оценена величиной $C(\varepsilon+\sqrt{\sigma}+\nu)$. Тогда из этой оценки и свойств решений задач (6.1) и будет следовать теорема 3.

По определению $Q_{\varepsilon}$ и $P_{\varepsilon}$ равны нулю на $\Omega_{1}^{\varepsilon} \times(0, T)$. Следовательно, выполнены соотношения (3.11)-(3.14) и равенство (4.4). Соответствующие интегралы по $\Omega$, содержашие $\nabla r_{a}, J_{1}$ и $J_{3}$ (из (3.11)), не сушественны в $(3.9)$, поскольку, например, для интеграла с $\nabla r_{a}$ выполнено неравенство (3.15). 
Выделяя сингулярное слагаемое в (4.4), введем обозначение (4.5) и определим $v_{3}$ из равенства (4.6). Непосредственно из (1.6), (3.5), (3.7), (4.1), (4.4) и леммы 5 заключаем, что выполнено неравенство (3.18). При выводе этого неравенства использовано, что $v_{3}$ не содержит градиентов от $Q_{\varepsilon}$ и $P_{\varepsilon}$, оценки которых могут зависеть от $\nu$. Как и в $(4.7)$, оценивается в $(3.9)$ интеграл по $\Omega_{0}^{\varepsilon}$, содержаший $k_{\varepsilon}^{\sigma}$ :

$$
\int_{0}^{T}\left|\int_{\Omega_{0}^{\varepsilon}}\left(k_{\varepsilon}^{\sigma} \nabla v_{a}\right) r d x\right| d t \leqslant C\left(\frac{\sigma}{\varepsilon \nu}+\sigma\right) \leqslant C \sqrt{\sigma}
$$

где учтены лемма 1 , неравенства (3.5) и (6.2), гладкости $v, g, f$ и тот факт, что $\nabla v_{a}-v_{2}$ не содержит градиентов от $Q_{\varepsilon}$ и $P_{\varepsilon}$.

Непосредственно из (1.6) и (4.5) следует, что

$$
\begin{gathered}
\int_{\Omega_{0}^{\varepsilon}}\left(A_{\varepsilon}^{\sigma} v_{2}, \nabla r\right) d x=\varepsilon \nu^{2} \int_{\Omega_{0}^{\varepsilon}}\left(\left(A_{0} \nabla_{y} P\right)_{\varepsilon} * f, \nabla r\right) d x \\
-\varepsilon \nu^{2} \int_{\Omega_{0}^{\varepsilon}}\left(\left(A_{0} \nabla_{y} Q\right)_{\varepsilon} *\left(v_{t}^{\prime}\right), \nabla r\right) d x .
\end{gathered}
$$

Используя первое равенство из (2.7) и начальные условия из (6.1), получаем равенства (4.10). Следовательно, учитьвая (3.11), (3.13), (3.15), (3.18), (6.4), (6.5) и (4.10), неравенство (3.9) можно переписать в виде (4.11) и выполнены соотношения (4.12), в которых величину $\vartheta$ следует заменить на $\nu^{2}$.

Используя (1.5), перепишем осредненное уравнение из (1.20) в виде

$$
\frac{\left\langle m_{1}\right\rangle_{\mathbf{1}}}{\theta_{1}} v_{t}^{\prime}-\frac{A}{\theta_{1}}\left(\nabla^{2} v+a \nabla v+b \nabla g\right)+\frac{K}{\theta_{1}} \nabla v+\frac{k_{a}}{\theta_{1}} v+\frac{k_{b}}{\theta_{1}} g-\frac{\left\langle r_{1}\right\rangle_{\mathbf{1}}}{\theta_{1}} f=0
$$

и введем обозначения (3.25) для функций, имеющих нулевое среднее на $Y_{1}$. Умножим (6.6) на $r$, проинтегрируем полученное соотношение по $\Omega_{1}^{\varepsilon}$ и учтем результат и равенство (3.22) в (4.11). Тогда, оценивая интегралы в (4.11), содержашие функции из (3.25), как и в $\S 3$ (см. (3.27)-(3.29)), неравенство (4.11) можно переписать в виде

$$
\begin{aligned}
E(r) \leqslant & C(\varepsilon+\sqrt{\sigma}+\nu)+C \int_{0}^{T} \mid \varepsilon \nu^{2} \int_{S_{\varepsilon}}\left(A_{0} \nabla_{y} P, \nu\right)_{\varepsilon} * f r d s \\
& -\varepsilon \nu^{2} \int_{S_{\varepsilon}}\left(A_{0} \nabla_{y} Q, \nu\right)_{\varepsilon} *\left(v_{t}^{\prime}\right) r d s \mid d t .
\end{aligned}
$$

Далее следует учесть, что $\nu$ является малым параметром, и построить главные члены асимптотики решений задач (6.1). Это построение будет проведено позже, а на данный момент отметим следуюшее следствие из этих построений.

ЛЕмма 10. Для решений задач (6.1) выполнены неравенства

$$
\begin{aligned}
& \|Q-1\|_{L^{\infty}\left(0, T ; L^{1}\left(Y_{0}\right)\right)}+\left\|P-\frac{r_{0}}{m_{0}}\right\|_{L^{\infty}\left(0, T ; L^{1}\left(Y_{0}\right)\right)} \leqslant C \nu, \\
& \|Q-1\|_{L^{\infty}\left(0, T ; L^{2}\left(Y_{0}\right)\right)}^{2}+\left\|P-\frac{r_{0}}{m_{0}}\right\|_{L^{\infty}\left(0, T ; L^{2}\left(Y_{0}\right)\right)}^{2} \leqslant C \nu .
\end{aligned}
$$


Из этой леммы и условий (1.9) следует, что

$$
\left\|\left\langle m_{0} Q-m_{0}\right\rangle_{\mathbf{0}}\right\|_{L^{1}(0, T)}+\left\|\left\langle m_{0} P-r_{0}\right\rangle_{\mathbf{0}}\right\|_{L^{1}(0, T)} \leqslant C \nu .
$$

Действительно, используя свойства интеграла Лебега, получаем

$$
\left|\left\langle m_{0} P-r_{0}\right\rangle_{\mathbf{0}}\right| \leqslant\left\langle m_{0}\left|P-\frac{r_{0}}{m_{0}}\right|\right\rangle_{\mathbf{0}} \leqslant C\left\|P-\frac{r_{0}}{m_{0}}\right\|_{L^{1}\left(Y_{0}\right)},
$$

и интегрирование этих неравенств по $t \in(0, T)$ доказывает (6.9).

Таким образом, учитывая (6.9), лемму 5 , гладкости $f$ и $v$, неравенство (6.7) можно переписать в виде

$$
\begin{aligned}
E(r) & \leqslant C(\varepsilon+\sqrt{\sigma}+\nu) \\
& +C \int_{0}^{T} \mid \int_{\Omega_{1}^{\varepsilon}}\left(\frac{\left\langle m_{0} P-r_{0}\right\rangle_{\mathbf{0}}}{\theta_{1}}\right)_{\varepsilon} *\left(f_{t}^{\prime}\right) r d x+\varepsilon \nu^{2} \int_{S_{\varepsilon}}\left(A_{0} \nabla_{y} P, \nu\right)_{\varepsilon} * f r d s \\
& -\int_{\Omega_{1}^{\varepsilon}}\left(\frac{\left\langle m_{0} Q-m_{0}\right\rangle_{\mathbf{0}}}{\theta_{1}}\right)_{\varepsilon} *\left(v_{t t}^{\prime \prime}\right) r d x-\varepsilon \nu^{2} \int_{S_{\varepsilon}}\left(A_{0} \nabla_{y} Q, \nu\right)_{\varepsilon} *\left(v_{t}^{\prime}\right) r d s \mid d t .
\end{aligned}
$$

Это неравенство фактически совпадает с неравенством (4.14), и поэтому выполнены соотношения (4.15)-(4.21), в которых величину $\vartheta$ следует заменить на $\nu^{2}$.

Таким образом, доказано неравенство

$$
E(r) \leqslant C(\varepsilon+\sqrt{\sigma}+\nu) .
$$

По определению $Q_{\varepsilon}$ и $P_{\varepsilon}$ равны нулю на $\Omega_{1}^{\varepsilon}$. Следовательно,

$$
\max _{0 \leqslant t \leqslant T}\|u-v\|_{L^{2}\left(\Omega_{1}^{\varepsilon}\right)}^{2} \leqslant E(r)+C \varepsilon \leqslant C(\varepsilon+\sqrt{\sigma}+\nu)
$$

в силу представления (6.3) и неравенств (3.5). Аналогично, получаем

$$
\begin{aligned}
& \max _{0 \leqslant t \leqslant T} \| u-v-\left(P_{\varepsilon}-\left(\frac{r_{0}}{m_{0}}\right)_{\varepsilon}\right) * f-\left(\frac{r_{0}}{m_{0}}\right)_{\varepsilon} * f \\
& \quad+\left(Q_{\varepsilon}-1\right) *\left(v_{t}^{\prime}\right)+1 *\left(v_{t}^{\prime}\right) \|_{L^{2}\left(\Omega_{0}^{\varepsilon}\right)}^{2} \leqslant C(\varepsilon+\sqrt{\sigma}+\nu) .
\end{aligned}
$$

Используя лемму 10 , ограниченность $\Omega_{0}^{\varepsilon}$, гладкости $f$ и $v$, имеем

$$
\max _{0 \leqslant t \leqslant T}\left\|\left(P_{\varepsilon}-\left(\frac{r_{0}}{m_{0}}\right)_{\varepsilon}\right) * f\right\|_{L^{2}\left(\Omega_{0}^{\varepsilon}\right)}^{2}+\max _{0 \leqslant t \leqslant T}\left\|\left(Q_{\varepsilon}-1\right) *\left(v_{t}^{\prime}\right)\right\|_{L^{2}\left(\Omega_{0}^{\varepsilon}\right)}^{2} \leqslant C \nu
$$

(см. доказательство леммы 5). Тогда из (6.11) заключаем, что

$$
\max _{0 \leqslant t \leqslant T}\left\|u-v-\left(\frac{r_{0}}{m_{0}}\right)_{\varepsilon} * f+1 *\left(v_{t}^{\prime}\right)\right\|_{L^{2}\left(\Omega_{0}^{\varepsilon}\right)}^{2} \leqslant C(\varepsilon+\sqrt{\sigma}+\nu) .
$$

Кроме того, в силу определений (2.6) выполнены равенства

$$
\left(\frac{r_{0}}{m_{0}}\right)_{\varepsilon} * f=\left(\frac{r_{0}}{m_{0}}\right)_{\varepsilon} \int_{0}^{t} f d t, \quad 1 *\left(v_{t}^{\prime}\right)=v-w
$$

которые вместе с (6.10) и (6.12) доказывают теорему 3. 
ДокАЗАТЕЛЬСТво ЛЕммы 10. Рассмотрим, например, начально-краевую задачу для $P$ из (6.1). В силу леммы 3 сушествует единственное решение $P \in$ $L^{2}\left(0, T ; H_{P}^{1}(Y)\right)$ этой задачи и

$$
\|P\|_{L^{\infty}\left(0, T ; L^{2}\left(Y_{0}\right)\right)}^{2}+\nu^{2}\left\|\nabla_{y} P\right\|_{L^{2}\left(0, T ; L^{2}\left(Y_{0}\right)^{n}\right)}^{2} \leqslant C .
$$

В этом неравенстве и далее постоянная $C$ не зависит от $\nu$.

Пусть заданы такие вектор-функция $V_{\nu} \in L^{2}\left(0, T ; L^{2}\left(Y_{0}\right)^{n}\right)$ и функция $W_{\nu} \in$ $L^{2}\left(0, T ; L^{2}\left(Y_{0}\right)\right)$, что

$$
\left\|V_{\nu}\right\|_{L^{2}\left(0, T ; L^{2}\left(Y_{0}\right)^{n}\right)}+\left\|W_{\nu}\right\|_{L^{2}\left(0, T ; L^{2}\left(Y_{0}\right)\right)} \leqslant C .
$$

Определим функцию $P_{\nu}(t, y)$ как 1-периодическое решение начально-краевой задачи на $Y_{0}$ :

$$
\begin{aligned}
& m_{0}\left(P_{\nu}\right)_{t}^{\prime}-\nu^{2} \operatorname{div}_{y}\left(A_{0} \nabla_{y} P_{\nu}\right)=\nu^{2} \operatorname{div}_{y}\left(V_{\nu}\right)+\nu W_{\nu} \quad \text { в } Y_{0} \times(0, \infty), \\
& P_{\nu}=0 \text { на } S \times(0, \infty),\left.\quad P_{\nu}\right|_{t=0}=\frac{r_{0}}{m_{0}} \text { в } Y_{0} .
\end{aligned}
$$

Тогда, повторяя доказательство леммы 1, заключаем, что

$$
\left\|P-P_{\nu}\right\|_{L^{\infty}\left(0, T ; L^{2}\left(Y_{0}\right)\right)}^{2}+\nu^{2}\left\|\nabla_{y}\left(P-P_{\nu}\right)\right\|_{L^{2}\left(0, T ; L^{2}\left(Y_{0}\right)^{n}\right)}^{2} \leqslant C \nu^{2} .
$$

Кроме того, из компактности $Y_{0}$ следует, что

$$
\left\|P-P_{\nu}\right\|_{L^{\infty}\left(0, T ; L^{1}\left(Y_{0}\right)\right)} \leqslant C\left\|P-P_{\nu}\right\|_{L^{\infty}\left(0, T ; L^{2}\left(Y_{0}\right)\right)} \leqslant C \nu .
$$

Таким образом, лемму 10 достаточно доказать для решения $P_{\nu}$ задачи (6.15). Другими словами, в доказательстве леммы 10 первое уравнение для $P$ из (6.1) допускает возмушения $\nu W_{\nu}$ и $\nu^{2} \operatorname{div}_{y}\left(V_{\nu}\right)$, удовлетворяюшие (6.14).

Будем искать решение задачи $(6.1)$ в виде $P=U+\left(r_{0} / m_{0}\right)$. Тогда для $U=P-$ $\left(r_{0} / m_{0}\right)$ имеем начально-краевую задачу на $Y_{0}$ :

$$
\begin{aligned}
& m_{0} U_{t}^{\prime}-\nu^{2} \operatorname{div}_{y}\left(A_{0} \nabla_{y} U\right)=\nu^{2} \operatorname{div}_{y}\left(A_{0} \nabla_{y}\left(\frac{r_{0}}{m_{0}}\right)\right) \text { в } Y_{0} \times(0, \infty), \\
& U=\frac{r_{0}}{m_{0}} \text { на } S \times(0, \infty),\left.\quad U\right|_{t=0}=0 \quad \text { в } Y_{0} .
\end{aligned}
$$

В силу неравенств (6.16), (6.17) и условий теоремы 3 можно считать в этой задаче, что $A_{0} \nabla_{y}\left(r_{0} / m_{0}\right)=0$. Из неравенства (6.13) и условий теоремы 3 также следует, что

$$
\|U\|_{L^{\infty}\left(0, T ; L^{2}\left(Y_{0}\right)\right)}^{2}+\nu^{2}\left\|\nabla_{y} U\right\|_{L^{2}\left(0, T ; L^{2}\left(Y_{0}\right)^{n}\right)}^{2} \leqslant C .
$$

Рассмотрим $Y_{0}$ как компактное $n$-мерное многообразие с гранищей $S$ класса $C^{3}$ (в силу условий теоремы 3 ). По определению найдется такое конечное разбиение единицы $\left\{\varphi_{\kappa}\right\}$, что для каждого $\kappa$ пересечение $\operatorname{supp}\left(\varphi_{\kappa}\right) \cap Y_{0}$ диффеоморфно $\mathbb{R}^{n}$ или полупространству $\mathbb{R}_{+}^{n}=\left\{z=\left(z_{1}, \ldots, z_{n}\right), z_{n}>0\right\}$.

Для каждого $\kappa$ определим $U_{\kappa}$ как решение начально-краевой задачи:

$$
\begin{aligned}
& m_{0}\left(U_{\kappa}\right)_{t}^{\prime}-\nu^{2} \operatorname{div}_{y}\left(A_{0} \nabla_{y} U_{\kappa}\right)=0 \text { в } Y_{0} \times(0, \infty), \\
& U_{\kappa}=\varphi_{\kappa} \frac{r_{0}}{m_{0}} \text { на } S \times(0, \infty),\left.\quad U_{\kappa}\right|_{t=0}=0 \text { в } Y_{0} .
\end{aligned}
$$


По определению $\sum_{\kappa} \varphi_{\kappa}=1$. Следовательно, $U=\sum_{\kappa} U_{\kappa}$ в силу единственности решений задачи $(6.18)$. Кроме того, если $\operatorname{supp}\left(\varphi_{\kappa}\right)$ не пересекается с $S$, то $U_{\kappa}=0$ в соответствии с (6.20).

Фиксируем $\kappa$ и определим $U_{\kappa}^{\kappa}=\varphi_{\kappa} U$. Тогда, умножая (6.18) на $\varphi_{\kappa}$, получим для $U_{\kappa}^{\kappa}$ начально-краевую задачу:

$$
\begin{aligned}
& m_{0}\left(U_{\kappa}^{\kappa}\right)_{t}^{\prime}-\nu^{2} \operatorname{div}_{y}\left(A_{0} \nabla_{y} U_{\kappa}^{\kappa}\right)=-\nu^{2}\left[\operatorname{div}_{y}\left(A_{0} \nabla_{y}\right), \varphi_{\kappa}\right] U \text { в } Y_{0} \times(0, \infty), \\
& U_{\kappa}^{\kappa}=\varphi_{\kappa} \frac{r_{0}}{m_{0}} \text { на } S \times(0, \infty),\left.\quad U_{\kappa}^{\kappa}\right|_{t=0}=0 \text { в } \quad Y_{0} .
\end{aligned}
$$

Здесь $\left[\operatorname{div}_{y}\left(A_{0} \nabla_{y}\right), \varphi_{\kappa}\right]$ обозначает коммутатор дифференциального оператора $\operatorname{div}_{y}\left(A_{0} \nabla_{y}\right)$ и оператора умножения на функцию $\varphi_{\kappa}$. Несложно проверить, что этот коммутатор является дифференциальным оператором первого порядка (с непрерывными коэффициентами в силу условий теоремы 3 ). Кроме того, этот коммутатор умножается на $\nu^{2}$, и $U$ удовлетворяет неравенству (6.19). Поэтому можно считать этот коммутатор нулевым в (6.21) (в смысле выполнения неравенств (6.16) и (6.17)).

Таким образом, можно считать, что $U_{\kappa}^{\kappa}=U_{\kappa}$, и ввести новые координаты $z \in \mathbb{R}_{+}^{n}$ в задаче (6.20). Воспользуемся для этого известными формулами замены координат в дифференциальных операторах второго порядка (см., например, [15]). Тогда задачу (6.20) в координатах $z$ можно записать в виде

$$
\begin{aligned}
& \left(U_{\kappa}\right)_{t}^{\prime}-\nu^{2} a_{m} \partial_{n}^{2} U_{\kappa}-\nu^{2} H\left(z, \partial_{z}\right) U_{\kappa}=0 \text { в } \mathbb{R}_{+}^{n} \times(0, \infty), \\
& U_{\kappa}=r_{\kappa} \text { на }\left\{z_{n}=0\right\} \times(0, \infty),\left.\quad U_{\kappa}\right|_{t=0}=0 \text { в } \mathbb{R}_{+}^{n},
\end{aligned}
$$

где $r_{\kappa}=\varphi_{\kappa} r_{0} / m_{0}, \partial_{n}$ обозначает частную производную по $z_{n}$, коэффициент $a_{m}$ принадлежит $C^{2}\left(\overline{\mathbb{R}}_{+}^{n}\right)$ и удовлетворяет неравенствам

$$
\alpha \leqslant a_{m}(z) \leqslant \beta \text { для } z \in \overline{\mathbb{R}}_{+}^{n}
$$

в силу неравенств $(1.7),(1.9)$ и условий теоремы 3 . Кроме того, $H\left(z, \partial_{z}\right)$ обозначает дифференциальный оператор второго порядка по $z$ (с непрерывными ограниченными коэффициентами в силу условий теоремы 3 ), но этот оператор содержит только производные первого порядка по $z_{n}$.

Используя формулу Тейлора, коэффициент $a_{m}$ представим в виде $a_{m}=a_{m}^{0}+$ $z_{n} a_{m}^{1}$ для $z_{n} \in[0,1]$, где $a_{m}^{0}=a_{m}$ при $z_{n}=0$. Далее, полагая $a_{m}^{1}=\left(a_{m}-a_{m}^{0}\right) / z_{n}$ для $z_{n} \in[1, \infty)$, имеем

$$
a_{m}=a_{m}^{0}+z_{n} a_{m}^{1} \quad \text { для } z \in \overline{\mathbb{R}}_{+}^{n},
$$

где $a_{m}^{0}$ и $a_{m}^{1}-$ непрерьвные ограниченные на $\overline{\mathbb{R}}_{+}^{n}$ функции, коэффициент $a_{m}^{0}$ принадлежит $C^{2}\left(\mathbb{R}^{n-1}\right)$ и удовлетворяет неравенствам (6.23).

Определим функцию $U_{\kappa}^{0}(t, z)$ как решение начально-краевой задачи на $\mathbb{R}_{+}^{n}$ :

$$
\begin{aligned}
& \left(U_{\kappa}^{0}\right)_{t}^{\prime}-\left(\frac{h^{2}}{4}\right) \partial_{n}^{2} U_{\kappa}^{0}=0 \text { в } \mathbb{R}_{+}^{n} \times(0, \infty), \\
& U_{\kappa}^{0}=r_{\kappa} \text { на }\left\{z_{n}=0\right\} \times(0, \infty),\left.\quad U_{\kappa}^{0}\right|_{t=0}=0 \quad \text { в } \mathbb{R}_{+}^{n},
\end{aligned}
$$


где $h^{2} / 4=\nu^{2} a_{m}^{0}$. Решение этой задачи задается равенствами

$$
\begin{aligned}
U_{\kappa}^{0} & =\frac{2 r_{\kappa}}{h \sqrt{\pi t}} \int_{-\infty}^{0} \exp \left(-\frac{\left(z_{n}-\xi\right)^{2}}{h^{2} t}\right) d \xi=-\frac{2 r_{\kappa}}{\sqrt{\pi}} \int_{\infty}^{z_{n}} \exp \left(-\frac{s^{2}}{h^{2} t}\right) d\left(\frac{s}{h \sqrt{t}}\right) \\
& =\frac{2 r_{\kappa}}{\sqrt{\pi}} \int_{\frac{z_{n}}{h \sqrt{t}}}^{\infty} e^{-\tau^{2}} d \tau=r_{\kappa} \operatorname{erfc}\left(\frac{z_{n}}{h \sqrt{t}}\right),
\end{aligned}
$$

где $\operatorname{erfc}(\cdot)$ обозначает специальную функцию, которую принято называть дополнительным интегралом вероятностей (см., например, [18]).

Действительно, так определенное $U_{\kappa}^{0}$ удовлетворяет первому уравнению из (6.25), что проверяется непосредственным дифференцированием под интегралом в первом равенстве из (6.26) (см., например, [19]). Далее следует учесть, что $\operatorname{erfc}(0)=1$ и $\operatorname{erfc}(\tau)$ стремится к нулю при $\tau \rightarrow \infty$ (см., например, [18, гл. 7]). Тогда из последнего равенства в (6.26) заключаем, что $U_{\kappa}^{0}$ удовлетворяет граничным и начальным условиям из (6.25). Таким образом, формула (6.26) определяет классическое решение задачи (6.25).

Оценим норму этого решения в $L^{\infty}\left(0, T ; L^{1}\left(\mathbb{R}_{+}^{n}\right)\right)$ и $L^{\infty}\left(0, T ; L^{2}\left(\mathbb{R}_{+}^{n}\right)\right)$. Напомним, что $h=2 \nu \sqrt{a_{m}^{0}}$. Следовательно,

$$
\begin{aligned}
& \int_{0}^{\infty}\left|U_{\kappa}^{0}\right| d z_{n}=\left|r_{\kappa}\right| \int_{0}^{\infty} \operatorname{erfc}\left(\frac{z_{n}}{h \sqrt{t}}\right) d z_{n} \\
& \quad=\left|r_{\kappa}\right| h \sqrt{t} \int_{0}^{\infty} \operatorname{erfc}(\tau) d \tau \leqslant \nu c\left(z_{0}\right) \sqrt{T} \int_{0}^{\infty} \operatorname{erfc}(\tau) d \tau
\end{aligned}
$$

Здесь и далее $z_{0}=\left(z_{1}, \ldots, z_{n-1}\right)$ и $c\left(z_{0}\right)$ обозначает ограниченные функции с компактным носителем на $\mathbb{R}^{n-1}$. Значение последнего интеграла в (6.27) выражается через значения гамма-функции и поэтому ограничено постоянной (см., например, $[18$, гл. $7, \S 7.4])$. Интегрируя неравенство $(6.27)$ по $\mathbb{R}^{n-1}$ и учитывая свойства функции $c\left(z_{0}\right)$, получаем

$$
\left\|U_{\kappa}^{0}\right\|_{L^{\infty}\left(0, T ; L^{1}\left(\mathbb{R}_{+}^{n}\right)\right)} \leqslant C \nu .
$$

Используя, что $\operatorname{erfc}(\tau) \leqslant 1$ для неотрицательных $\tau$, имеем

$$
\int_{0}^{\infty}\left(U_{\kappa}^{0}\right)^{2} d z_{n} \leqslant r_{\kappa}^{2} \int_{0}^{\infty} \operatorname{erfc}\left(\frac{z_{n}}{h \sqrt{t}}\right) d z_{n} \leqslant \nu c\left(z_{0}\right) \sqrt{T} \int_{0}^{\infty} \operatorname{erfc}(\tau) d \tau .
$$

Интегрируя эти неравенства по $\mathbb{R}^{n-1}$, заключаем, что

$$
\left\|U_{\kappa}^{0}\right\|_{L^{\infty}\left(0, T ; L^{2}\left(\mathbb{R}_{+}^{n}\right)\right)}^{2} \leqslant C \nu .
$$

Из неравенств (6.28) и (6.30) будет следовать лемма 10, если доказать соответствующую близость $U_{\kappa}^{0}$ к решению задачи (6.22). Для доказательства этого утверждения оценим некоторые нормы от $U_{\kappa}^{0}$.

Введем обозначение $\operatorname{erfc}^{(1)}(\tau)=(\operatorname{erfc}(\tau))_{\tau}^{\prime}=-(2 / \sqrt{\pi}) e^{-\tau^{2}}$. Тогда, дифференцируя (6.26), имеем $\partial_{n} U_{\kappa}^{0}=\left(r_{\kappa} / h \sqrt{t}\right) \operatorname{erfc}^{(1)}\left(z_{n} / h \sqrt{t}\right)$, и поэтому

$$
\begin{aligned}
\int_{0}^{\infty}\left(\partial_{n} U_{\kappa}^{0}\right)^{2} d z_{n} & =\frac{r_{\kappa}^{2}}{h^{2} t} \int_{0}^{\infty} \operatorname{erfc}^{(1)}\left(\frac{z_{n}}{h \sqrt{t}}\right)^{2} d z_{n} \\
& =\frac{r_{\kappa}^{2}}{h \sqrt{t}} \int_{0}^{\infty} \operatorname{erfc}^{(1)}(\tau)^{2} d \tau=\frac{c\left(z_{0}\right)}{\nu \sqrt{t}} \operatorname{erfc}(0)
\end{aligned}
$$


Интегрируя это равенство по $\mathbb{R}^{n-1}$ и затем по $(0, T)$, имеем

$$
\left\|\partial_{n} U_{\kappa}^{0}\right\|_{L^{2}\left(0, T ; L^{2}\left(\mathbb{R}_{+}^{n}\right)\right)}^{2} \leqslant \frac{C}{\nu} .
$$

Обозначим через $\partial_{0}$ одну из частных производных по $z_{1}, \ldots, z_{n-1}$. Тогда из (6.26) следует, что

$$
\begin{aligned}
\partial_{0} U_{\kappa}^{0} & =\left(\partial_{0} r_{\kappa}\right) \operatorname{erfc}\left(\frac{z_{n}}{h \sqrt{t}}\right)+\frac{r_{\kappa} z_{n}}{\nu \sqrt{t}} \operatorname{erfc}^{(1)}\left(\frac{z_{n}}{h \sqrt{t}}\right) \partial_{0}\left(\frac{1}{2 \sqrt{a_{m}^{0}}}\right) \\
& =\left(\partial_{0} r_{\kappa}\right) \operatorname{erfc}\left(\frac{z_{n}}{h \sqrt{t}}\right)+\frac{c\left(z_{0}\right) z_{n}}{h \sqrt{t}} \operatorname{erfc}^{(1)}\left(\frac{z_{n}}{h \sqrt{t}}\right) .
\end{aligned}
$$

Для оценки первого слагаемого в последнем равенстве из (6.33) достаточно повторить доказательство неравенств (6.29) и (6.30). Следовательно,

$$
\left\|\partial_{0} U_{\kappa}^{0}\right\|_{L^{\infty}\left(0, T ; L^{2}\left(\mathbb{R}_{+}^{n}\right)\right)}^{2} \leqslant C \nu,
$$

поскольку вьполнено равенство

$$
c^{2}\left(z_{0}\right) \int_{0}^{\infty}\left(\frac{z_{n}}{h \sqrt{t}} \operatorname{erfc}^{(1)}\left(\frac{z_{n}}{h \sqrt{t}}\right)\right)^{2} d z_{n}=\nu c_{1}\left(z_{0}\right) \sqrt{t} \int_{0}^{\infty}\left(\tau \operatorname{erfc}^{(1)}(\tau)\right)^{2} d \tau
$$

где последний интеграл ограничен постоянной (см., например, [18, гл. $7, \S 7.4])$.

Введем также обозначение $\operatorname{erfc}^{(2)}(\tau)=\left(\operatorname{erfc}^{(1)}(\tau)\right)_{\tau}^{\prime}=(-2 \tau) \operatorname{erfc}^{(1)}(\tau)$. Тогда из (6.33) следует, что

$$
\partial_{0}^{2} U_{\kappa}^{0}=c_{1}\left(z_{0}\right) \operatorname{erfc}\left(\frac{z_{n}}{h \sqrt{t}}\right)+\frac{c_{2}\left(z_{0}\right) z_{n}}{h \sqrt{t}} \operatorname{erfc}^{(1)}\left(\frac{z_{n}}{h \sqrt{t}}\right)+\frac{c_{3}\left(z_{0}\right) z_{n}^{2}}{h^{2} t} \operatorname{erfc}^{(2)}\left(\frac{z_{n}}{h \sqrt{t}}\right) .
$$

Для оценки первых двух слагаемых в этом равенстве достаточно повторить доказательство неравенств (6.30) и (6.34). Следовательно,

$$
\left\|\partial_{0}^{2} U_{\kappa}^{0}\right\|_{L^{\infty}\left(0, T ; L^{2}\left(\mathbb{R}_{+}^{n}\right)\right)}^{2} \leqslant C \nu,
$$

поскольку вьполнено равенство

$$
c_{3}^{2}\left(z_{0}\right) \int_{0}^{\infty}\left(\frac{z_{n}^{2}}{h^{2} t} \operatorname{erfc}^{(2)}\left(\frac{z_{n}}{h \sqrt{t}}\right)\right)^{2} d z_{n}=\nu c_{4}\left(z_{0}\right) \sqrt{t} \int_{0}^{\infty}\left(\tau^{3} \operatorname{erfc}^{(1)}(\tau)\right)^{2} d \tau
$$

где последний интеграл ограничен постоянной (см., например, $[18$, гл. $7, \S 7.4])$.

Аналогично получаем $\partial_{n}^{2} U_{\kappa}^{0}=\left(r_{\kappa} / h^{2} t\right) \operatorname{erfc}^{(2)}\left(z_{n} / h \sqrt{t}\right)$, и поэтому

$$
\begin{aligned}
\int_{0}^{\infty}\left(z_{n} a_{m}^{1} \partial_{n}^{2} U_{\kappa}^{0}\right)^{2} d z_{n} & \leqslant \frac{c_{1}\left(z_{0}\right)}{h^{2} t} \int_{0}^{\infty}\left(\frac{z_{n}}{h \sqrt{t}} \operatorname{erfc}^{(2)}\left(\frac{z_{n}}{h \sqrt{t}}\right)\right)^{2} d z_{n} \\
& =\frac{c_{1}\left(z_{0}\right)}{h \sqrt{t}} \int_{0}^{\infty}\left(\tau \operatorname{erfc}^{(2)}(\tau)\right)^{2} d \tau \\
& \leqslant \frac{c_{2}\left(z_{0}\right)}{\nu \sqrt{t}} \int_{0}^{\infty}\left(\tau^{2} \operatorname{erfc}^{(1)}(\tau)\right)^{2} d \tau,
\end{aligned}
$$


где последний интеграл ограничен постоянной (см., например, $[18$, гл. $7, \S 7.4])$. Интегрируя эти неравенства по $\mathbb{R}^{n-1}$ и затем по $(0, T)$, заключаем, что

$$
\left\|z_{n} a_{m}^{1} \partial_{n}^{2} U_{\kappa}^{0}\right\|_{L^{2}\left(0, T ; L^{2}\left(\mathbb{R}_{+}^{n}\right)\right)}^{2} \leqslant \frac{C}{\nu} .
$$

Пусть $R_{\kappa}=U_{\kappa}-U_{\kappa}^{0}$, где $U_{\kappa}$ - решение задачи $(6.22)$ и $U_{\kappa}^{0}$ - решение задачи (6.25). Тогда, учитывая (6.24) и уравнения из $(6.25)$, получим для $R_{\kappa}$ начально-краевую задачу:

$$
\begin{aligned}
& \left(R_{\kappa}\right)_{t}^{\prime}-\nu^{2} a_{m} \partial_{n}^{2} R_{\kappa}-\nu^{2} H\left(z, \partial_{z}\right) R_{\kappa}=\nu W_{\kappa} \quad \text { в } \mathbb{R}_{+}^{n} \times(0, \infty), \\
& R_{\kappa}=0 \text { на }\left\{z_{n}=0\right\} \times(0, \infty),\left.\quad R_{\kappa}\right|_{t=0}=0 \quad \text { в } \mathbb{R}_{+}^{n},
\end{aligned}
$$

где $W_{\kappa}=\nu z_{n} a_{m}^{1} \partial_{n}^{2} U_{\kappa}^{0}+\nu H\left(z, \partial_{z}\right) U_{\kappa}^{0}$. Из неравенств $(6.30),(6.32),(6.34)-(6.36)$ следует, что для $W_{\kappa}$ выполнена оценка

$$
\left\|W_{\kappa}\right\|_{L^{2}\left(0, T ; L^{2}\left(\mathbb{R}_{+}^{n}\right)\right)} \leqslant C
$$

Аналогичная оценка выполнена для $m_{0} W_{\kappa}$ в силу условий (1.9). Поэтому, возврашаясь в задаче (6.37) к координатам $y \in Y_{0}$, получаем для $R_{\kappa}$ начально-краевую задачу (6.15), в которой $V_{\nu}=0$ и $W_{\nu}$ удовлетворяет (6.14).

Таким образом, в координатах $y \in Y_{0}$ конечная сумма

$$
P=\sum_{\kappa} \varphi_{\kappa}\left(U_{\kappa}^{0}+\frac{r_{0}}{m_{0}}\right)
$$

определяет главный член асимптотики решения задачи (6.1) для $P$ и неравенства (6.28) и (6.30) доказывают лемму 10.

ЗАмЕчАниЕ 8 . Для функции $\operatorname{erfc}(\tau)$ выполнено следующее асимптотическое разложение:

$$
\operatorname{erfc}(\tau)=\frac{e^{-\tau^{2}}}{\tau \sqrt{\pi}}\left[1+O\left(\frac{1}{\tau^{2}}\right)\right] \text { при } \tau \rightarrow \infty
$$

(см., например, $\left[18\right.$, гл. 7]). Кроме того, $\operatorname{erfc}\left(\tau_{1}\right) \leqslant \operatorname{erfc}\left(\tau_{2}\right)$ для таких неотрицательных $\tau_{1}$ и $\tau_{2}$, что $\tau_{2} \leqslant \tau_{1}$. Фиксируем некоторое положительное $z_{n}^{0}$. Тогда в силу (6.39) найдутся такие положительные постоянные $C, c$ и $\nu_{0}$, что

$$
\left|U_{\kappa}^{0}\right| \leqslant \nu C e^{-\left(c z_{n} / \nu\right)^{2}} \text { для } \nu \leqslant \nu_{0} \quad \text { и } \quad z_{n}^{0} \leqslant z_{n} .
$$

Таким образом, функция $P(t, y)-r_{0}(y) / m_{0}(y)$ достаточно быстро убывает при удалении точки $y$ от гранищы $S$.

Предполагая бо́льшую гладкость коэфффициентов и границы включений, чем в теореме 3 , можно построить и дальнейшие члены асимптотического разложения решений задачи (6.1), следуя основным идеям метода Вишика-Люстерника (см., например, [20]). 


\section{§7. Доказательство следствий}

Известно [4], что в предположениях следствий 1,2 или 3 решение задачи (1.4) сушествует и удовлетворяет неравенству

$$
\|u\|_{L^{\infty}\left(0, T ; L^{2}(\Omega)\right)}^{2} \leqslant C\left(\|f\|_{L^{2}\left(0, T ; L^{2}(\Omega)\right)}^{2}+\|g\|_{L^{2}\left(0, T ; L^{2}(\Omega)\right)}^{2}+\|w\|_{L^{2}(\Omega)}^{2}\right),
$$

где постоянная $C$ не зависит от $\varepsilon$ и $\sigma$ (см. доказательство леммы 1$)$. Отметим, что несколько более общая оценка леммы 1 выполнена при дополнительном условии $w \in H_{0}^{1}(\Omega)$ (см., например, [1], [4]).

Аналогично, в предположениях следствий 1 или 3 решения осредненных задач (1.16) и (1.20) сушествуют и удовлетворяют неравенству

$$
\|v\|_{L^{\infty}\left(0, T ; L^{2}(\Omega)\right)}^{2} \leqslant C\left(\|f\|_{L^{2}\left(0, T ; L^{2}(\Omega)\right)}^{2}+\|g\|_{L^{2}\left(0, T ; L^{2}(\Omega)\right)}^{2}+\|w\|_{L^{2}(\Omega)}^{2}\right) .
$$

Из результатов работы [6] (см. также лемму 10) следует аналогичное утверждение для осредненной задачи (1.19) и

$$
\begin{aligned}
& \|v\|_{L^{\infty}\left(0, T ; L^{2}(\Omega)\right)}^{2}+\left\|v_{t}^{\prime}\right\|_{L^{2}\left(0, T ; L^{2}(\Omega)\right)}^{2} \\
& \quad \leqslant C\left(\|f\|_{L^{2}\left(0, T ; L^{2}(\Omega)\right)}^{2}+\|g\|_{L^{2}\left(0, T ; H^{1}(\Omega)\right)}^{2}\right),
\end{aligned}
$$

если выполнены предположения следствия 2 .

Известно [1], что множества $C_{0}^{\infty}(\Omega)$ и $C_{0}^{\infty}((0, T) \times \Omega)$ плотны в сепарабельных пространствах $L^{2}(\Omega)$ и $L^{2}\left(0, T ; L^{2}(\Omega)\right)$ соответственно. Поэтому найдутся такие последовательности $\left\{f_{s}\right\},\left\{g_{s}\right\} \subset C_{0}^{\infty}((0, T) \times \Omega)$ и $\left\{w_{s}\right\} \subset C_{0}^{\infty}(\Omega)$, что

$$
\left\|f-f_{s}\right\|_{L^{2}\left(0, T ; L^{2}(\Omega)\right)}^{2}+\left\|g-g_{s}\right\|_{L^{2}\left(0, T ; L^{2}(\Omega)\right)}^{2}+\left\|w-w_{s}\right\|_{L^{2}(\Omega)}^{2} \leqslant \delta_{s} .
$$

Здесь и далее $\delta_{s}$ достаточно мало при достаточно больших $s$.

Для каждого $s$ обозначим через $u_{s}$ решение задачи (1.4), в которой $f, g$ и $w$ заменены на $f_{s}, g_{s}$ и $w_{s}$ соответственно. Аналогично, обозначим через $v_{s}$ решение одной из осредненных задач (1.16), (1.19) или (1.20), в которых $f, g$ и $w$ заменены на $f_{s}, g_{s}$ и $w_{s}$. Используя неравенства (7.1)-(7.4) и линейность рассматриваемых задач, заключаем, что

$$
\left\|u-u_{s}\right\|_{L^{\infty}\left(0, T ; L^{2}(\Omega)\right)}^{2} \leqslant C \delta_{s}, \quad\left\|v-v_{s}\right\|_{L^{\infty}\left(0, T ; L^{2}(\Omega)\right)}^{2} \leqslant C \delta_{s},
$$

где постоянная $C$ не зависит от $\varepsilon, \sigma$ и $s$. Кроме того, имеем неравенство

$$
\left\|\left(v-v_{s}\right)_{t}^{\prime}\right\|_{L^{2}\left(0, T ; L^{2}(\Omega)\right)}^{2} \leqslant C \delta_{s},
$$

если выполнены предположения следствия 2.

ДоКАЗАТЕЛЬСТво СЛЕДСТвИя 1. Для $u_{s}$ выполнены условия теоремы 1. Разумеется, постоянная $C$ из неравенства этой теоремы может зависеть от $s$. Поэтому из неравенств (7.5) и неравенства треугольника следует, что

$$
\begin{gathered}
\|u-v\|_{L^{\infty}\left(0, T ; L^{2}(\Omega)\right)}^{2}=\left\|u-u_{s}+u_{s}-v_{s}+v_{s}-v\right\|_{L^{\infty}\left(0, T ; L^{2}(\Omega)\right)}^{2} \\
\leqslant C \delta_{s}+C\left\|u_{s}-v_{s}\right\|_{L^{\infty}\left(0, T ; L^{2}(\Omega)\right)}^{2} \leqslant C \delta_{s}+C_{s}\left(\varepsilon+\sigma+\frac{\varepsilon^{2}}{\sigma}\right) .
\end{gathered}
$$


Для произвольного $\delta$ можно выбрать $s$ так, чтобы $C \delta_{s}<\delta / 2$. В силу условия (1.1) найдутся такие $\varepsilon(\delta)$ и $\sigma(\delta)$, что $C_{s}\left(\varepsilon+\sigma+\varepsilon^{2} / \sigma\right)<\delta / 2$ при $\varepsilon<\varepsilon(\delta)$ и $\sigma<\sigma(\delta)$. Таким образом, из неравенства (7.7) имеем

$$
\|u-v\|_{L^{\infty}\left(0, T ; L^{2}(\Omega)\right)}^{2}<\delta \text { при } \varepsilon<\varepsilon(\delta) \text { и } \sigma<\sigma(\delta),
$$

что доказывает следствие 1.

ДОКАЗАТЕЛЬСТВО СЛЕДСТВИЯ 2. В предположениях следствия 2 свертки $P_{\varepsilon} * f$ и $Q_{\varepsilon} *\left(v_{t}^{\prime}\right)$ могут быть не определены как элементы пространства $L^{\infty}\left(0, T ; L^{2}(\Omega)\right)$, поскольку не всегда определено поточечное умножение двух функций из $L^{2}(\Omega)$ (см. определения (2.6)). В силу (4.1) для этих сверток выполнены только неравенства

$$
\begin{gathered}
\left\|\left(Q_{\varepsilon} *\left(v_{t}^{\prime}\right)\right)(t)\right\|_{L^{1}(\Omega)} \leqslant \int_{0}^{T}\left\|Q_{\varepsilon}(t-\tau) v_{t}^{\prime}(\tau)\right\|_{L^{1}(\Omega)} d \tau \\
\leqslant C \int_{0}^{T}\left\|Q_{\varepsilon}(t-\tau)\right\|_{L^{2}(\Omega)}\left\|v_{t}^{\prime}(\tau)\right\|_{L^{2}(\Omega)} d \tau \leqslant C\left\|v_{t}^{\prime}\right\|_{L^{2}\left(0, T ; L^{2}(\Omega)\right)}, \\
\left\|\left(P_{\varepsilon} * f\right)(t)\right\|_{L^{1}(\Omega)} \leqslant C\|f\|_{L^{2}\left(0, T ; L^{2}(\Omega)\right)}
\end{gathered}
$$

(см. доказательство леммы 5). Поэтому $P_{\varepsilon} * f, Q_{\varepsilon} *\left(v_{t}^{\prime}\right) \in L^{\infty}\left(0, T ; L^{1}(\Omega)\right)$ в силу неравенства (7.3) и предположений следствия 2.

Используя неравенства (7.4)-(7.6) и теорему 2 , получаем

$$
\begin{aligned}
& \left\|u-v-P_{\varepsilon} * f+Q_{\varepsilon} *\left(v_{t}^{\prime}\right)\right\|_{L^{\infty}\left(0, T ; L^{1}(\Omega)\right)}^{2} \\
& \leqslant \\
& \quad C \delta_{s}+C\left\|u_{s}-v_{s}-P_{\varepsilon} * f_{s}+Q_{\varepsilon} *\left(v_{s}\right)_{t}^{\prime}\right\|_{L^{\infty}\left(0, T ; L^{1}(\Omega)\right)}^{2} \\
& \quad+C\left\|P_{\varepsilon} *\left(f_{s}-f\right)\right\|_{L^{\infty}\left(0, T ; L^{1}(\Omega)\right)}^{2}+C\left\|Q_{\varepsilon} *\left(v_{s}-v\right)_{t}^{\prime}\right\|_{L^{\infty}\left(0, T ; L^{1}(\Omega)\right)}^{2} \\
& \leqslant C \delta_{s}+C_{s}\left(\varepsilon+\sqrt{\sigma}+\left|\vartheta-\frac{\sigma}{\varepsilon^{2}}\right|\right)+C \delta_{s},
\end{aligned}
$$

где учтены неравенства $(7.8)$, в которых $v_{t}^{\prime}$ и $f$ заменены на $\left(v_{s}-v\right)_{t}^{\prime}$ и $f_{s}-f$ соответственно.

Для произвольного $\delta$ можно выбрать $s$ так, чтобы $C \delta_{s}<\delta / 4$. В силу условия (1.2) найдутся такие $\varepsilon(\delta)$ и $\sigma(\delta)$, что $C_{s}\left(\varepsilon+\sqrt{\sigma}+\left|\vartheta-\sigma / \varepsilon^{2}\right|\right)<\delta / 2$ при $\varepsilon<\varepsilon(\delta)$ и $\sigma<\sigma(\delta)$. Таким образом, из неравенства (7.9) имеем

$$
\left\|u-v-P_{\varepsilon} * f+Q_{\varepsilon} *\left(v_{t}^{\prime}\right)\right\|_{L^{\infty}\left(0, T ; L^{1}(\Omega)\right)}^{2}<\delta \text { при } \varepsilon<\varepsilon(\delta) \text { и } \sigma<\sigma(\delta),
$$

что доказывает сходимость второго слагаемого из утверждения следствия 2.

По определению $Q_{\varepsilon}$ и $P_{\varepsilon}$ равны нулю на $\Omega_{1}^{\varepsilon} \times(0, T)$. Поэтому, учитывая неравенства (7.5) и теорему 2 , для завершения доказательства следствия 2 достаточно повторить доказательство следствия 1. 
ДОКАЗАТЕЛЬСТВО СЛЕДСТВИЯ 3. Заметим, что

$$
\left\|\left(\frac{r_{0}}{m_{0}}\right)_{\varepsilon} \int_{0}^{t}\left(f-f_{s}\right) d t\right\|_{L^{2}(\Omega)} \leqslant C \int_{0}^{T}\left\|f-f_{s}\right\|_{L^{2}(\Omega)} d t \leqslant C \sqrt{\delta_{s}}
$$

в силу (7.4) и неравенства Коши-Буняковского. Кроме того, имеем неравенство

$$
\begin{aligned}
& \left\|u-\chi_{1}^{\varepsilon} v-\chi_{0}^{\varepsilon} w-\chi_{0}^{\varepsilon}\left(\frac{r_{0}}{m_{0}}\right)_{\varepsilon} \int_{0}^{t} f d t\right\|_{L^{\infty}\left(0, T ; L^{2}(\Omega)\right)}^{2} \\
& \quad \leqslant\|u-v\|_{L^{\infty}\left(0, T ; L^{2}\left(\Omega_{1}^{\varepsilon}\right)\right)}^{2}+\left\|u-w-\left(\frac{r_{0}}{m_{0}}\right)_{\varepsilon} \int_{0}^{t} f d t\right\|_{L^{\infty}\left(0, T ; L^{2}\left(\Omega_{0}^{\varepsilon}\right)\right)}^{2} .
\end{aligned}
$$

Поэтому, учитывая неравенства $(7.4),(7.5),(7.10)$ и теорему 3 , для доказательства следствия 3 достаточно повторить доказательство следствия 1.

ЗАМЕЧАНИЕ 9. Используя подходящие аппроксимации коэффициентов, можно вывести утверждение следствия 3 при более слабых предположениях на коэффициенты задачи (1.4). Аналогично, утверждения следствий 1-3 выполнены при более слабых предположениях на гладкость границ $\partial \Omega, \partial F_{0}$ и $\partial F_{1}$. Однако такой путь доказательства этих утверждений является достаточно длинным, и проще дать непосредственные доказательства, которые будут приведены где-нибудь в другом месте.

\section{Список литературы}

1. Лионс Ж.-Л., Мадженес Э. Неоднородные граничные задачи и их приложения. М.: Мир, 1971.

2. Бахвалов Н. С., Панасенко Г. П. Осреднение процессов в периодических средах. М.: Наука, 1984.

3. Жиков В.В., Козлов С. М., Олейник О.А. Усреднение дифференциальных операторов. М.: Физматлит, 1993.

4. Ладыженская О. А., Солонников В. А., Уральчева Н. Н. Линейные и квазилинейные уравнения параболического типа. М.: Наука, 1967.

5. Сандраков Г. В. Осреднение линеаризованной системы гидродинамики с малой вязкостью и скорость звука в смесях. Препринт № 178. М.: ОВМ АН СССР, 1987.

6. Hornung U., Showalter R.E. Diffusion models for fractured media // J. Math. Analysis and Applications. 1990. V. 147. № 1. P. 67-80.

7. Сандраков Г.В. Принципы осреднения уравнений с быстроосциллирующими коэффициентами // Матем. сб. 1989. Т. 180. № 12. С. 1643-1690.

8. Панасенко Г. П. Многокомпонентное осреднение процессов в сильно неоднородных структурах // Матем. сб. 1990. Т. 181. №1. С. 134-142.

9. Бахвалов Н. С., Эглит М. Э. О предельном поведении периодических сред с мягкомодульными включениями // ЖВМ и МФ. 1995. Т. 35. №6. С. 905-917.

10. Хруслов Е. Я., Рост Г. Усреднение уравнения диффузии с коэффициентами, не удовлетворяющими условию равномерной эллиптичности // УМН. 1991. Т. 46. № 6. С. 189-190.

11. Hornung $U$., Jager $W$. Diffusion, convection, adsorption, and reaction of chemicals in porous media // J. Diff. Equat. 1991. V. 92. № 2. P. 199-225.

12. Arbogast T, Douglas J., Hornung U. Derivation of the double porosity model of single phase flow via homogenization theory // SIAM J. Math. Anal. 1990. V. 21. № 4. P. 823-836.

13. Сандраков Г. В. Диффузия в сильно неоднородной периодической среде // УМН. 1996. T. 51. № 5. C. 208-209.

14. Темам Р. Уравнения Навье-Стокса. М.: Мир, 1981.

15. Владимиров В. С. Уравнения математической физики. М.: Наука, 1988. 
16. Агранович M. C., Вишик M. И. Эллиптические задачи с параметром и параболические задачи общего вида // УМН. 1964. Т. 19. № 3. С. 53-161.

17. Хилле Э., Филлипс Р. Функциональный анализ и полугруппы. М.: ИЛ, 1962.

18. Абрамович М., Стиган И. Справочник по специальным функциям с формулами, графиками и таблицами. М.: Наука, 1979.

19. Годунов C. К. Уравнения математической физики. М.: Наука, 1979.

20. Треногин В. А. Развитие и применение метода Люстерника-Вишика // УМН. 1970. T. 25. № 4. C. 123-156.

Механико-математический факультет, кафедра Вычислительной математики

Поступило в редакцию

Московский государственный университет

им. М. В. Ломоносова

E-mail: sandrako@compmath.math.msu.su

ПРИМЕЧАНИЕ ПРИ КОРРЕКТУРЕ. Отметим несколько работ, не попавших в список литературы по различным причинам, в которых рассматриваются близкие проблемы и приведены утверждения о сходимости решений в соответствующих пространствах.

Allaire G. Homogenization and two-scale convergence // SIAM J. Math. Anal. 1992. V. 23. P. 1482-1518.

Bourgeat A., Mikelić A., Piatnitski A. Modele de double porosité aléatoire // Comp. Rend. Acad. Sci. Paris. Ser. 1. 1998. V. 327. P. 99-104.

Pankratov L. S. Homogenization of semilinear parabolic equations with asymptotically degenerating coefficients // Матем. физика, анализ, геометрия. 1998. Т. 5. С. 250-273. 